\title{
FIELD DETECTION DEVICE OF REACTIVE OXYGEN SPECIES
}

\author{
A Thesis \\ presented to \\ the Faculty of California Polytechnic State University, \\ San Luis Obispo
}

\author{
In Partial Fulfillment \\ of the Requirements for the Degree \\ Master of Science in Biomedical Engineering
}

by

Danielle Lee Cowgill

December 2013 
(C) 2013

Danielle Lee Cowgill

ALL RIGHTS RESERVED 


\section{COMMITTEE MEMBERSHIP}

TITLE:

AUTHOR:

DATE SUBMITTED:

COMMITTEE CHAIR:

COMMITTEE MEMBER:

COMMITTEE MEMBER:
Field Detection Device of Reactive Oxygen Species

Danielle Lee Cowgill

December 2013

Lily Laiho, $\mathrm{PhD}$.

Associate Professor of Biomedical

Engineering

Richard Savage, PhD.

Professor of Biomedical Engineering

Kristen O'Halloran Cardinal, $\mathrm{PhD}$. Associate Professor of Biomedical Engineering 


\section{ABSTRACT \\ Field Detection Device of Reactive Oxygen Species \\ Danielle Lee Cowgill}

Traumatic brain injuries (TBIs) contribute to approximately $30.5 \%$ of all injury related deaths in the United States per year and approximately 1.7 million TBIs per year occur as an isolated injury or other injury. Military personnel are exposed to TBIs and have less availability to resources traditionally used to diagnose a TBI. Reactive oxygen species (ROS) play a large role in secondary traumatic brain damage, especially lipid peroxidation, following TBIs. One of the principle and most studied byproducts of lipid peroxidation is malondialdehyde (MDA). The focus of this paper was to create an initial proof-of-concept field diagnostic device to detect ROS, or constituents, following a TBI. The device was divided into three main sections: blood separation, sample separation, and detection. This thesis report examined the blood and sample separation sections of the device; however the blood separation was not developed due to university restrictions. Pre-fabricated microfluidic chips and a confocal microscope were used for the separation. Pressure was first used to drive the fluid through the channel to observe functionality of the chip with 10 um microspheres and then a MDA solution once the spheres were successful. When fluid successfully flowed through the channels with pressure, voltage was then applied to the channels to create a capillary electrophoresis (CE) system. The CE system was tested with the spheres first and then the MDA solution upon successful demonstration of voltage-driven flow. Analysis of the images was performed using ImageJ software in attempt to determine the velocity of the spheres and MDA. The results of the system appeared to successfully drive the MDA with voltage-driven flow after waiting for the residual effects of the applied pressure to reduce. However, any conclusions require more experimental data. 


\section{ACKNOWLEDGMENTS}

I would like to thank Dr. Laiho for all of her patience and guidance through this entire project. Your understanding and encouragement kept me going, even meeting me the week after graduation. Thank you for believing in me and giving me confidence. Finally, thank you for helping me finish this project even when I was hundreds of miles away had to call and email so many times.

I am very thankful for my committee members for being willing to be a part of my committee. Dr. Savage, your enthusiasm for materials engineering was infectious and had me looking forward to your Friday morning labs. Dr. Kristen O'Halloran Cardinal, your knowledge and teaching methods always pushed me to work harder and reminded me how much I enjoy learning.

Thank you to QL+ for sponsoring my project and allowing me to work on such an interesting and exciting area of research. I wanted to work on a project for you when I started Cal Poly and saw the lab being built. The work you do deserves more recognition and I'm honored to have had the ability to work with you.

Finally, I would like to thank my family and friends who bore the worst of me through all of my years in college. No matter what curveballs life threw me, you all were there to cheer me on and pick me back up when I needed it the most. My sister, Elizabeth, you have always been a great role model and I love how we can talk about random things all the time. Lastly, I would like to thank my dad, the pep talks, the boxes of cookies in the mail, and the reminder of how there is always a silver lining in any situation has helped shape me into the person I am today. 


\section{TABLE OF CONTENTS}

LIST OF TABLES ….......................................................................... viii

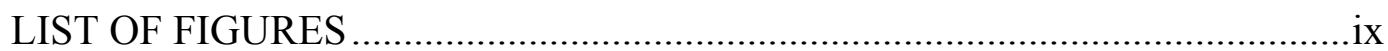

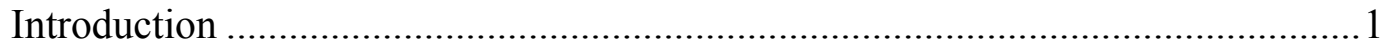

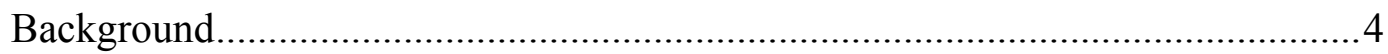

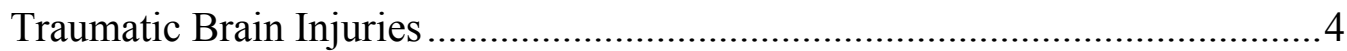

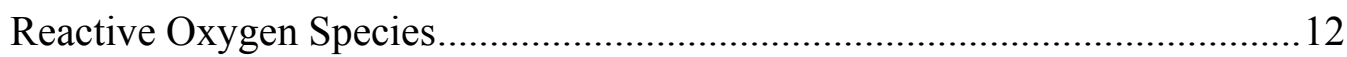

Capillary Electrophoresis........................................................................20

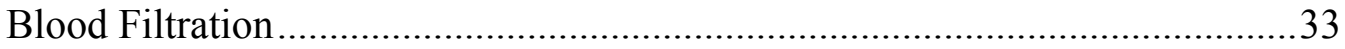

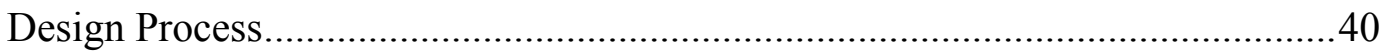

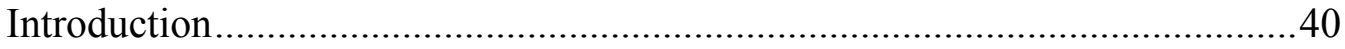

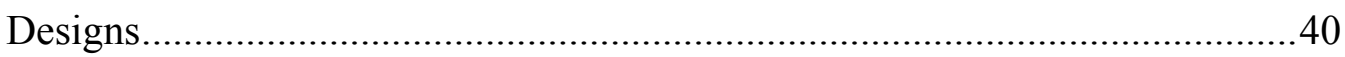

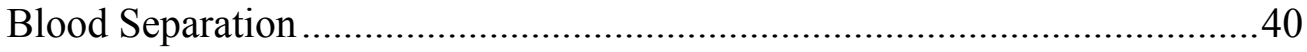

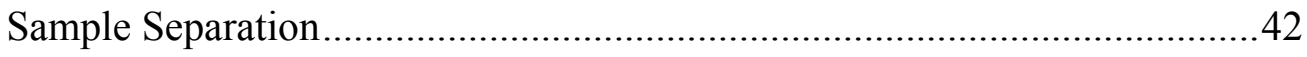

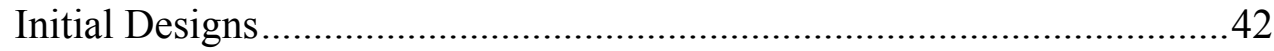

Final Design of Proof of Concept.........................................................49

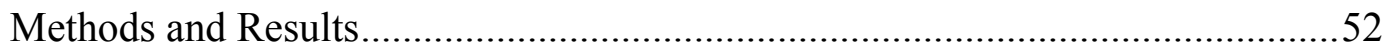

Pressure Driven Flow on Confocal Microscope ............................................52

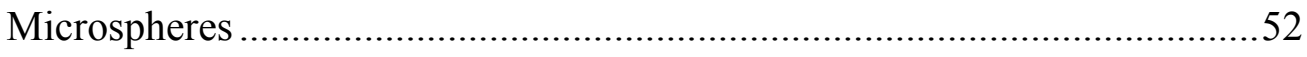

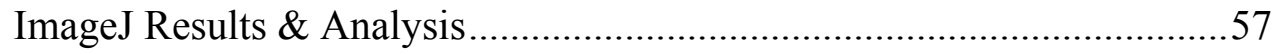

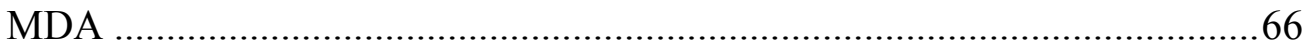

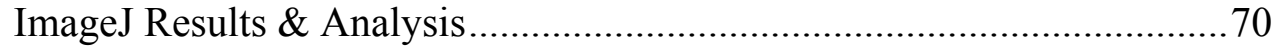

Model of Microchip Capillary Electrophoresis System ...............................78

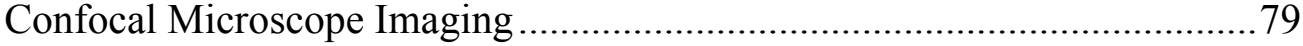

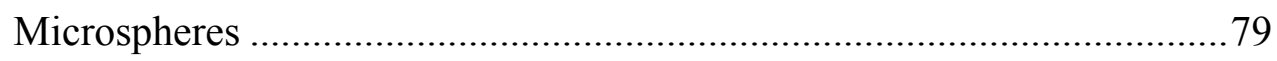

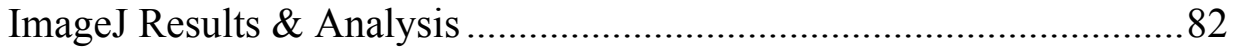

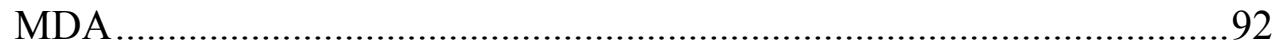

ImageJ Results \& Analysis ....................................................... 93

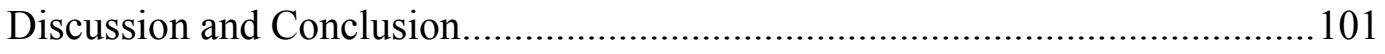

Pressure-Driven Flow on Confocal Microscope..........................................101

Voltage-Driven Flow on Confocal Microscope............................................. 103

Future Work and Recommendations .............................................................. 107

Pressure-Driven Flow on Confocal Microscope..........................................107

Voltage-Driven Flow on Confocal Microscope............................................ 108 


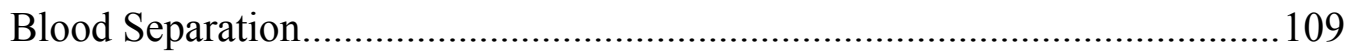

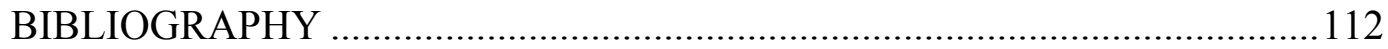




\section{LIST OF TABLES}

Table 1. Parameters used in various studies using CE to detect MDA in

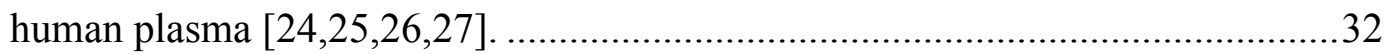

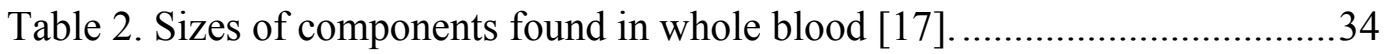

Table 3. Cost of Products from Translume [35] ..................................................49

Table 4. Table of labeled items found on Figure 21 of prototype........................50

Table 5. Table of experimental values for pressure-driven flow of $10 \mathrm{um}$

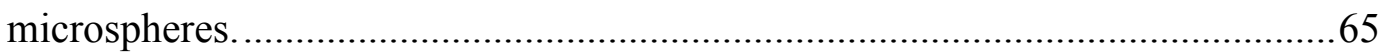

Table 6. Table of approximated values for pressure-driven flow of MDA

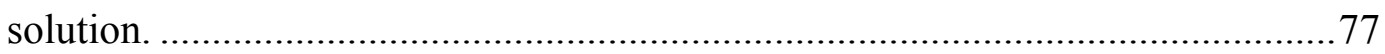

Table 7. Table of values for voltage-driven flow of $10 \mathrm{um}$ microspheres. ............89

Table 8. Variables used to calculate velocity of voltage-driven microspheres. ....90

Table 9. Table of values for voltage-driven flow of $10 \mathrm{um}$ microspheres. ...........91

Table 10. Table of values for voltage-driven flow of MDA solution. ..................99

Table 11. Variables used to calculate velocity of voltage-driven MDA. ..............99 


\section{LIST OF FIGURES}

Figure 1. Flow chart of factors that are associated with the three main types of battlefield traumatic brain injuries [30] ...........................................................

Figure 2. Simplified pathway describing production of ROS in a neuron [5]. .......7 Figure 3. Electron structures of some common reactive oxygen species

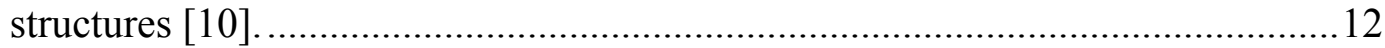

Figure 4. Balance required to maintain oxidative homeostasis [9] ......................13

Figure 5. Two hypotheses of formation of MDA A) predicting that MDA is derived from prostaglandin-like endoperoxides from methyl-linoleate during times of stressful conditions and B) MDA is produced by beta-scission or by reaction of the final acrolein radical with a hydroxyl radical [13]....

Figure 6. A) Relationship between time and MDA levels in patients with TBI [2]; B) MDA levels in jugular and peripheral blood over 48 hours [6], C) MDA levels in poor and good outcome groups over 48 hours [6].

Figure 7. Reaction between MDA and TBA to form TBARS and the corresponding excitation and emission spectrums [29].

Figure 8. Basic schematic design of capillary electrophoresis system [21] .........23

Figure 9. Table-top CE system used to detect charged molecules found in a sample (Cal Poly, Dairy Science Center) 25

Figure 10. Sample end of table-top CE system with capillary and cathode electrode extending into vials of samples and buffers on the sample tray $(\mathrm{Cal}$ Poly, Dairy Science Center).

Figure 11. Waste platform of table-top CE system with capillary and anode electrode extending into vials for waste (Cal Poly, Dairy Science Center). .........27

Figure 12. Typical layout for microchip CE device [21].

Figure 13. Setup and functionality of chip-based electrokinetic injection for CE system [21].

Figure 14. Examples of chip-based CE systems including (A) the $\mu$ ChemLab and (B) a system created by Becker et al [21].

Figure 15. Diagram of an example microchip design used to separate whole blood in Jiang experiment [20].

Figure 16. Sketch of a brainstormed idea of a CE system using single-use cartridges. 
Figure 17. Drawing of a brainstorm of a simplified CE system design consisting of various valves and tanks.

Figure 18. Drawing of a brainstorm of a simplified CE system design with an external blood separation system housed in a simple housing container.

Figure 19. Drawing of the pre-fabricated microfluidic chips purchased from Translume [35].

Figure 20. Chip with attached female luer lock connectors [35].

Figure 21. Prototype with main components labeled.

Figure 22. Pressure-driven system used to validate microfluidic chip on confocal microscope.

Figure 23. (Top) AutoCAD drawing of chip holder used to cut the acrylic.

(Bottom) Completed chip holding fixture with lip to hold chip while fixture is held by stage of confocal microscope.

Figure 24. Creating substacks from larger stacks in order to get desired frames for another stack using the Make Substack setting...................................58

Figure 25. Substack Maker dialogue box used to designate desired slices.

Figure 26. Setting the global scale of pixel to micron length to be applied to all images. 60

Figure 27. Creating a montage from a stack of images using the Make Montage setting. 60

Figure 28. Settings used to create montage of images from stack.

Figure 29. Montage of microspheres moving down channel of microfluidic chip due to pressure applied by a hand syringe

Figure 30. Measuring the distance spheres traveled between frames using ImageJ....

Figure 31. Set up of pressure-driven MDA test without chip holder and not on confocal microscope. 68

Figure 32. Image of empty channel after washing with deionized water. 70

Figure 33. Setting the global scale of pixel to micron length to be applied to all images

Figure 34. Creating substacks from larger stacks in order to get desired frames for another stack using the Make Substack setting..................................72

Figure 35. Substack Maker dialogue box used to designate desired slices. .72

Figure 36. Creating a montage from a stack of images using the Make Montage setting. 73

Figure 37. Settings used to create montage of images from stack. 
Figure 38. Montage of slices 120-140 of 200 image stack, showing MDA fluid in the channel, leaving the channel, and re-entering the channel.

Figure 39. Attempting to measure the distance spheres traveled between frames using ImageJ.

Figure 40. Labeled image of the CE system setup with the microchip that was used in the previous experiments.

Figure 41. Bench model of CE system setup on the confocal microscope with the voltage source.

Figure 42. Setting the global scale of pixel to micron length to be applied to all images

Figure 43. Creating substacks from larger stacks in order to get desired frames for another stack using the Make Substack setting. 84

Figure 44. Substack Maker dialogue box used to designate desired slices. 84

Figure 45. Creating a montage from a stack of images using the Make Montage setting. ..... 85

Figure 46. Settings used to create montage of images from stack.

Figure 47. Montage of images, in numerical order, demonstrating the flow of a single microsphere, circled in red, flowing down the channel, from right to left of the channel, in the same direction as the electric field.

Figure 48. Measuring the distance between microspheres flowing down the channel due to voltage-flow.

Figure 49. Voltage-driven flow of $10 \mathrm{um}$ spheres shifted by reversing the polarity of the electrodes with A) spheres flowing from left to right in the channel, B) reversing polarity so spheres flowed from right to left, and C) final polarity shift with spheres traveling from left to right. .91

Figure 50. Slice 1 of 200 showing the microchannel with no MDA present. .......94 Figure 51. Slice 20 of 200 showing the presence of MDA known by the increased intensity of the fluorescence in the channel.

Figure 52. Slice 23 of 200 with MDA present with parabolic flow leading and following the fluid.

Figure 53. Slice 24 of 200 with MDA present with parabolic flow following the fluid.

Figure 54. Slice 25 of 200 with MDA present in the top left corner of the channel in the region of interest, with a parabolic shape following the fluid .96 Figure 55. Slice 28 of 200 with MDA present in the microchannel as seen by the increased intensity of fluorescence. 
Figure 56. Measuring the distance traveled by the MDA solution in the

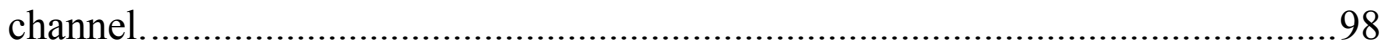

Figure 57. Possible design for microchip combining both whole blood separation and MDA detection................................................................ 110 
Introduction

Traumatic brain injuries (TBI) are a common injury especially in combat situations. Military personnel are exposed to all three types of TBI including closed head TBI, penetrating TBI, and $1^{\text {st }}$ degree explosive blast TBI (Figure 1) $[30]$.

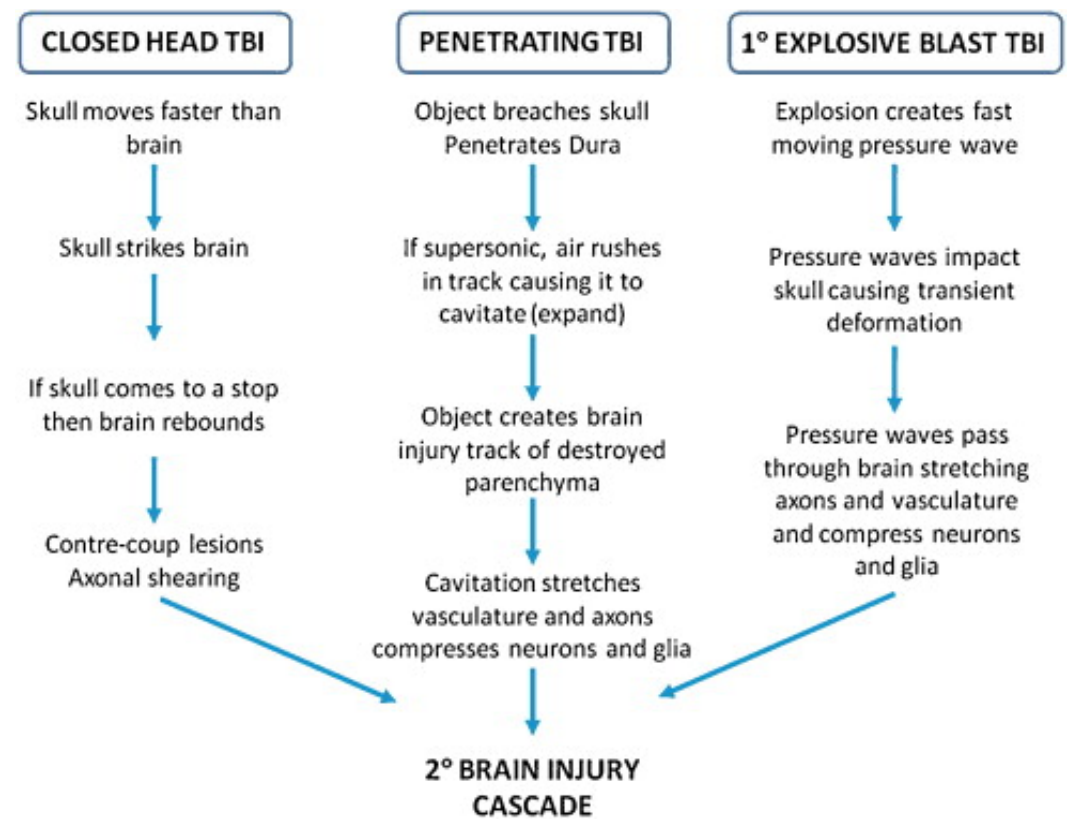

Figure 1. Flow chart of factors that are associated with the three main types of battlefield traumatic brain injuries [30].

It is impossible to prevent all TBIs so diagnosis and treatments are incredibly important to maintain quality of life for patients. With the war in Iraq, it is predicted that 15.2 to $22.8 \%$ of retuning service members will have a TBI [30]. Explosive blasts including improvised explosive devices (IEDs) are the predominant cause of combat related TBIs [30]. Protective equipment including 
helmets and body armor shield victims from some injuries like penetrating chest or abdomen wounds that would have been fatal [30]. However the lacking development with helmets has left the sides and face of military personnel exposed and vulnerable to serious injury [30]. By protecting more of the body, patients who previously perished due to other injuries are now surviving and suffering from TBIs that are sustained from the lack of protection to the cranium [30]. Currently, those injured in combat have procedures to aid in the diagnosis of severity of damage with verbal questions; however, the open-ended nature of the test can miss injuries that progressively deteriorate or do not test the area of the brain that is wounded. To fill this void in diagnosis, a system to analyze the physiological conditions of the patient needs to be developed.

Lab-on-a-chip is a current buzzword being used to describe devices that are in development for point-of-care diagnostics around the world. The military is in need of these types of devices as portability and ease of use are major design considerations leaving endless possibilities for such systems. A great application for one of these systems would be diagnosing TBIs in the field. There are many challenging aspects that arise with the creation of such devices. Many of these diagnostic tests require the use of raw biological samples to be processed. One of the most common samples is whole blood drawn from a patient. However, most tests require the whole blood to be separated to use the blood plasma. Additionally, the required information for many of the diseased states, like TBI, 
are lacking due to the numerous unknown factors of the pathophysiology and no standard protocol for testing for specific biomarkers.

One biomarker in assessment for TBI is malondialdehyde (MDA) [13]. It is one of the most studied reactive oxygen species (ROS) and is believed to be one of the contributing factors to neural tissue damage following TBI [13]. Many researchers have hypothesized that there is a correlation between MDA levels in human plasma and outcome following TBI [24, 25, 26, 27]. Many variables, like detection methodology and plasma treatments, have given rise to the many discrepancies in the relationship between TBI and MDA levels.

The focus of this paper is to create an initial proof-of-concept field diagnostic device to detect ROS, or constituents, following a TBI. Development of this device could help determine a relationship between MDA levels following TBI over various time points and in relation to the patient's outcome. The information gained by understanding the pathways and relationships, could greatly improve future treatment plans in the field of TBI for both military and civilians. 


\section{Background}

Traumatic Brain Injuries

Traumatic brain injuries (TBIs) contribute to approximately $30.5 \%$

of all injury related deaths in the United States per year [1]. Approximately 108 to 332 new cases per 100,000 people in the global population per year are hospitalized due to TBIs [2]. Early detection of TBIs, including point-of-care diagnostic tools, may help reduce unfavorable outcomes. Oxidative stress, an imbalance in the body resulting in an increase in oxidants over antioxidants, can be triggered by a TBI and can lead to secondary brain damage. Biomarkers such as reactive oxygen species may be used to determine the severity of TBI, assist in determining the best treatment plan for each patient, and help in developing new treatments for TBI.

There are approximately 1.7 million TBIs per year that occur as an isolated injury or other injury [1]. Globally, 39\% of patients with a severe TBI die from the injury, and $60 \%$ have an unfavorable outcome based on the Glasgow Outcome Scale [5]. The Glasgow Outcome Scale is used to objectively classify patients into the following five categories: dead, vegetative, severely disabled, moderately disabled, and good recovery [8]. By providing a more accurate diagnostic test, physicians can work to improve care for patients with unfavorable outcomes. Patients with unfavorable outcomes may have physical alterations in the brain can lead to functional short and long-term changes including thinking, 
sensation, language, and emotion [1]. It also leads to an increased risk of epilepsy, conditions like Alzheimer's and Parkinson's, and other brain disorders [1]. Survivors face prolonged care and rehabilitation including physical, cognitive, and psychological and generally die 2 to 3 times faster than the general public [5].

Improving diagnosis and treatments of TBI not only increases the quality of life for patients but also has strong economic benefits. Many relate TBIs with military applications such as falls or bodily collisions with objects following explosive blasts, penetrating wounds, and severe blows to the head with shrapnel or debris [3]. These events often lead to post-traumatic stress disorder (PTSD) that can be detrimental to patients. Civilians, particularly older adults, young children, and adolescent males, also have TBIs usually caused by falls, vehiclerelated collisions, violence, and sports injuries [3]. In the 2000, the United States spent an estimated $\$ 76.5$ billion due to direct costs, such as medical bills, and indirect costs, such as lost productivity of work, of TBIs. A conservative 2007 report in the United States estimated $\$ 396,331$ of lifetime costs following moderate to severe TBIs with the majority, $\$ 330,827$, due to disability and lost productivity at work and the rest, $\$ 65,504$, for medical care and rehabilitation [5].

The Center for Disease Control and Prevention (CDC) defines a traumatic brain injury (TBI) as a "bump, blow or jolt to the head, or penetrating head injury that disrupts the normal functions of the brain" [1]. TBIs can cause temporary 
dysfunction of brain cells leading to a range in severity of TBI from mild to severe [3]. In a mild TBI, there is a brief change in mental status or consciousness [1]; while a severe TBI is classified as an extended period of unconsciousness or amnesia after the injury [1] which can result in bruising, torn tissues, bleeding, and other physical damage to the brain that can result in long-term complications or death [3].

Mild TBIs are usually caused by concussions or alterations in brain function following a blow to the head [4]. Even though concussions are usually mild and brief, they can be damaging, and multiple concussions over time have been shown to lead to severe damage [4]. A more serious concussion, or contusion, can cause bruising of the brain and permanent neurological damage [4]. Severe TBIs often lead to subdural or subarachnoid hemorrhaging, bleeding from ruptured vessels following a blow to the head [4]. The blood that accumulates in the skull increases intracranial pressure leading to compression of the brain tissue, often resulting in death [4].

Pathologists have determined a simplified pathophysiological molecular pathway (Figure 2) to describe the cascade that follows a TBI [5]. The primary injury, such as a bomb blast, leads to an increase in concentration of extracellular glutamate that binds to neuronal receptors [5]. In turn there is an increase in intracellular calcium and sodium ion concentrations and an increase in 
concentration of extracellular potassium ions [5]. Due to these increased concentrations, the cell membrane of the neuron depolarizes and forces the mitochondria to malfunction and release reactive oxygen species (ROS) [5]. Rising levels of ROS disrupt the body's homeostasis resulting in oxidative stress, which damages the cell membrane, lipids, proteins, and DNA and ultimately leads to neuronal death [5]. Understanding pathways like this can lead to improved diagnostic techniques and treatments by targeting byproducts of TBI [5].

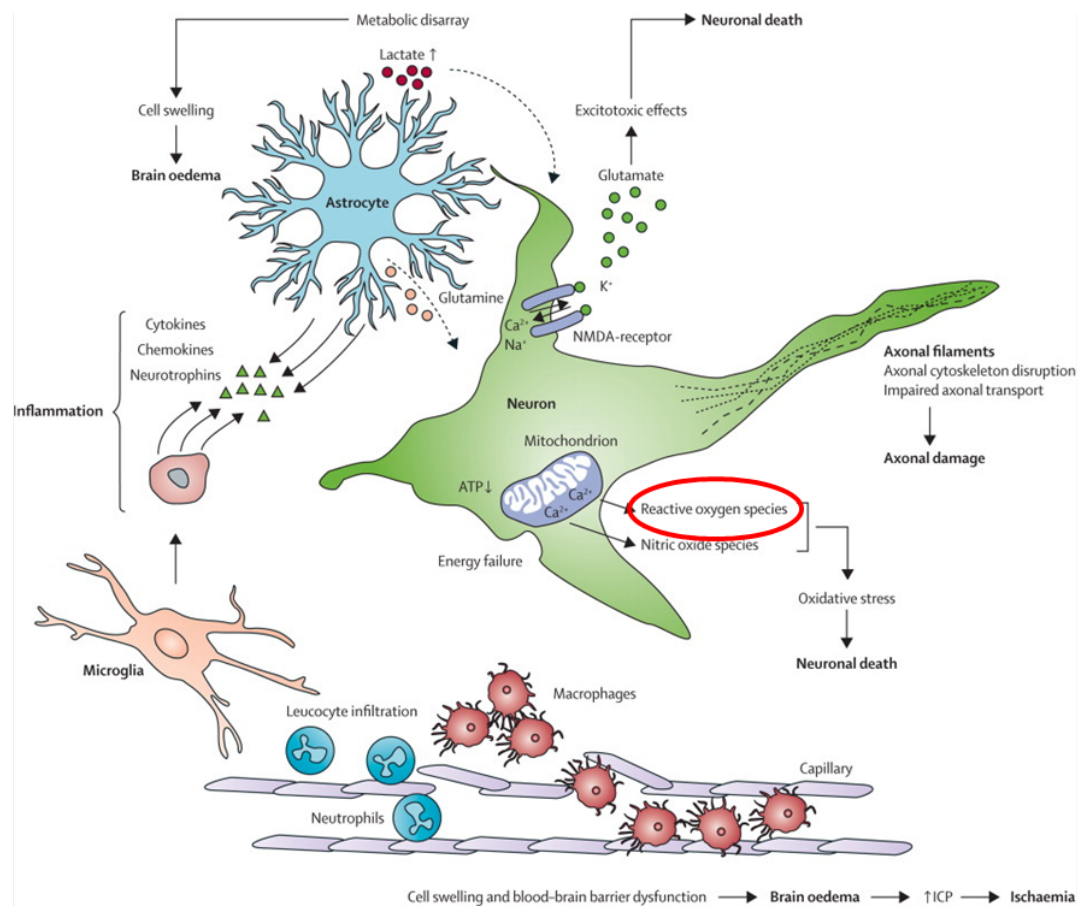

Figure 2. Simplified pathway describing production of ROS in a neuron [5].

Upon arrival to a trauma center, extensive TBI diagnosis is usually determined by imaging techniques, the Glasgow Coma Scale, and intracranial 
pressure monitoring [3]. The Glasgow Coma scale is an objective 15-point scale used to measure the motor response, verbal response, and eye opening response to help in determining the level for survival [8]. Imaging includes computed tomography (CT) scans that use $\mathrm{x}$-rays and magnetic resonance imaging (MRI) that use radio and magnetic rays [3]. The CT scans are to locate any visual regions of bleeding, clots, bruises, or swelling of the brain tissue [3]. MRI scans are used to produce detailed images in order to locate the prior named injuries [3]. In emergency situations, MRIs are not used, as they take longer to perform than that of CT scans, therefore they are often used in continuous monitoring of stabilized patients [3]. Intracranial monitoring is an invasive procedure in which probes are inserted into the skull to observe any swelling of the brain tissue that causes more damage to the brain [3]. All of these techniques require highly trained professionals and, in the case of the imaging, expensive and time consuming leading to more time for the secondary effects to cause more damage to the brain. In addition, TBIs have not been thoroughly studied as patients often have other life-threatening injuries that need to be treated before TBIs are considered or observed. Once a thorough diagnosis has been performed, the treatment plan is devised based on the results.

Current treatments are broken up into pre-hospital, non-surgical, surgical, and pharmalogical techniques. Pre-hospital treatments include inserting a flexible tube into the airway to aid the patient in breathing [5]. Currently there is no 
definitive data that demonstrates any positive pre-hospital treatments leaving room for research [5]. Non-surgical treatments include therapeutically induced hypothermia that can lead to a reduction in the formation of free radicals [5].

Results from patients who have undergone therapeutic hypothermia are conflicting with dependency on the time the patient undergoes hypothermia, the duration the patient is in a hypothermic state, the temperature at which the body is in a hypothermic state, and the rate at which the patient is returned to normal body temperature [5]. Additionally, there are many side effects to inducing hypothermia including clotting problems, increased chances of infection, insulin resistance, and cardiac rhythm problems [5]. Surgical treatments include decompressive crainectomy and implantation of stem cells [5]. Decompressive craniectomy is a surgical technique, where segments of the skull are removed in order to decrease intracranial pressure [5]. The procedure is standard for military blast injuries to the brain but there are many complications that frequently arise including excessive bleeding and cerebrospinal fluid buildup [5]. Stem cell transplantation is an experimental procedure in which cells are injected into the body via the blood stream to replace neural cells and decrease inflammation [5]. There are many difficulties with the procedure including timing of implantation and a procedure to directly inject the cells into the injured location without causing further damage [5]. Pharmacological techniques include immunosuppressive drugs, which stabilize the mitochondrial failure in the neuron, 
therefore reducing oxidative stress and lipid peroxidation in animal studies [5]. Many other drugs have similar concerns with positive results shown in animal studies but negative or no results shown in human studies [5]. There are some promising pharmaceutical treatments that are undergoing clinical trials to determine effectiveness [5]. All of these treatments show progress into creating successful results for patients however as seen from the negative results and many side effects, there are many avenues to pursue in treatment development for patients with TBI.

Military patients with TBI go through a diagnostic procedure called MACE (Military Acute Concussion Evaluation) [28]. MACE is designed to evaluate a patient who has received a concussion and determines their current clinical status, similar to the Glasgow Coma Scale used in civilian hospitals [28]. The 8-page evaluation has specific questions and directions in order to analyze the patient's orientation, immediate memory, neurological screening, concentration, and memory recall [28]. Answers are associated with a point scale that can demonstrate if a patient has received a concussion but is not a definitive guideline for diagnosis [28]. Certain questions are significant indicators for further evaluation and the answers can be used to show numerical data for assessment of the patient as they are improving in treatments [28]. Once a patient is evaluated based on MACE, the appropriate actions are taken either by allowing the patient to return to their military duties or to continue with a further diagnosis [28]. While 
the military uses civilian doctors to help develop processes that best fit the situation, they still have significant room for improvement as TBIs have been directly related to PTSD and other mental disorders along with death following the trauma. Field tests of physiological attributes could greatly aid in the diagnosis of TBIs for military personnel especially in combat areas.

In combat areas, patients are evaluated with the MACE procedure and are taken to concussion restoration centers at the military base [28]. However, many patients are injured during combat operations, which take place far from the military base [28]. When the patient is injured, their buddy asks them the questions following the MACE procedure and determines if further care is needed or if they can continue without further intervention [28]. If more evaluation is deemed necessary, the patient is returned to the base and taken to the concussion restoration center [28]. While this process is very successful for patients with mild concussions, patients with more severe cases require more complex diagnosis such as CT scans which usually are found at hospitals as the machines are large and expensive [28]. In the time required to take a patient to the hospital, further damage can occur to the brain before the diagnosis and can alter the outcome of the patient [28]. Patients often do not show any signs of injury and are not treated until their condition is more severe which can be difficult to treat [28]. With the key to treatment being early diagnosis, a field test system can improve outcomes of patients. 


\section{Reactive Oxygen Species}

Reactive oxygen species (ROS), or more commonly known as reactive molecules and free radicals, are found in the human body [9]. Free radicals have an unpaired electron and are very reactive [10]. A few ROS electron structures can be seen in Figure 3 below.

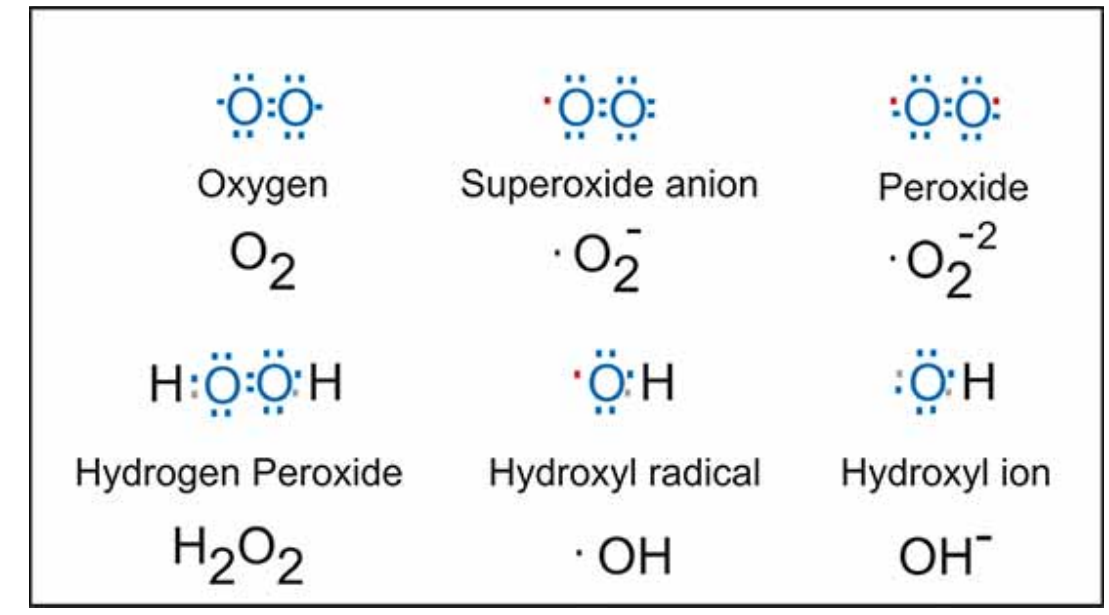

Figure 3. Electron structures of some common reactive oxygen species structures [10].

ROS are generated as a result of normal intracellular metabolism and a number of external factors [11]. Many ROS are byproducts of mitochondrial electron transport processes that are constantly occurring in the body [9]. The body maintains physiological homeostatic oxidation by regulating ROS production and maintaining their concentration to a non-damaging level via an enzymatic and non-enzymatic antioxidant system $[9,11]$. When the body receives an injury, the balance between production and removal shifts away from 
homeostasis and goes toward a pro-oxidative state (Figure 4) [9]. The shift to prooxidative state is caused by an increased production of ROS and a constant rate of removal of ROS leading to an increased concentration of ROS in the body [9]. Increased levels of ROS can lead to cell death or acceleration in aging and agerelated diseases as a result of random damage to proteins, lipids, and DNA [11]. Chronic pro-oxidative state is potentially linked to aging, chronic diseases, and cancer [9]. Detection and identification of ROS can be beneficial in order to better understand diseases when determining a cure.

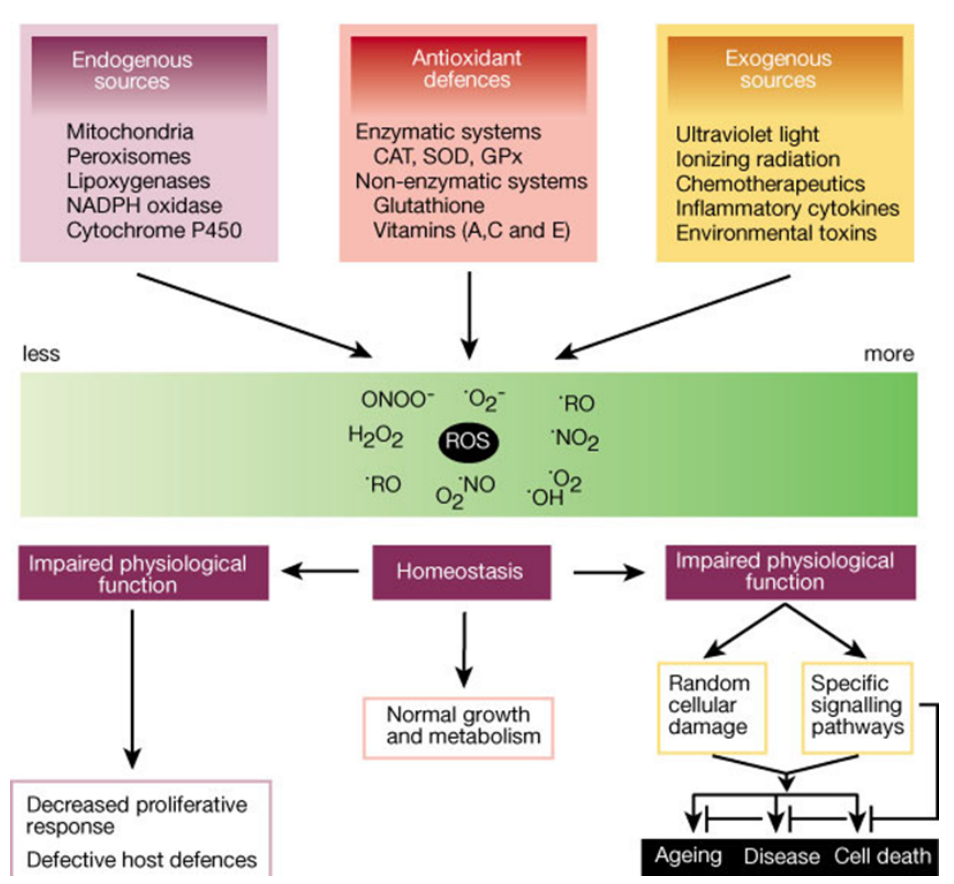

Figure 4. Balance required to maintain oxidative homeostasis [9].

ROS play a large role in secondary traumatic brain damage following TBI.

Secondary traumatic brain damage is associated with changes in basic chemical 
processes in the brain that then produce physical brain damage leading to difficulties with recovery [6]. Following TBI, ROS are produced from dysfunctional mitochondria as discussed in the pathway found in the previous section [6]. This increase in ROS leads to oxidative stress, which in turn can lead to oxidative damage to the lipids, also known as lipid peroxidation, and neuronal death [6]. The brain requires a high rate of oxygen consumption and it contains a high concentration of polyunsaturated fatty acids therefore it is highly susceptible to lipid peroxidation [6].

Lipid peroxidation or oxidative damage to lipids is the process of in which ROS take electrons from the lipids in cell membranes, and can ultimately lead to significant tissue damage [12]. In the brain, oxidative damage to lipids can alter the viscosity and ability of the cell membrane to allow particles to flow in and out of the cell leading to extensive tissue damage [6]. The process of lipid peroxidation can also produce many secondary products, including aldehydes, which cause further oxidative damage [13]. In vivo, ROS can be difficult to measure directly due to their short half-life; therefore many techniques measure the byproducts of ROS and lipid peroxidation in order to determine their role in diseased states such as TBI [6].

One of the principle and most studied byproducts of lipid peroxidation is malondialdehyde (MDA) [13]. The main source of MDA is the peroxidation of polyunsaturated fatty acids with two or more methylene-interrupted double bonds 
[13]. There are multiple hypotheses of how this occurs in vivo including the following two hypotheses. The first hypothesis described by Pryor and Stanley [14] predicts that MDA is derived from prostaglandin-like endoperoxides from methyl-linoleate during times of stressful conditions (Figure 5A) [13]. Another hypothesis described by Esterbauer [15] details a mechanism of successive hydroperoxide formations and beta-cleavage of the fatty acid chain to give a hydroperoxyaldehyde [13]. MDA is then produced by beta-scission or by reaction of the final acrolein radical with a hydroxyl radical (Figure 5B) [13].

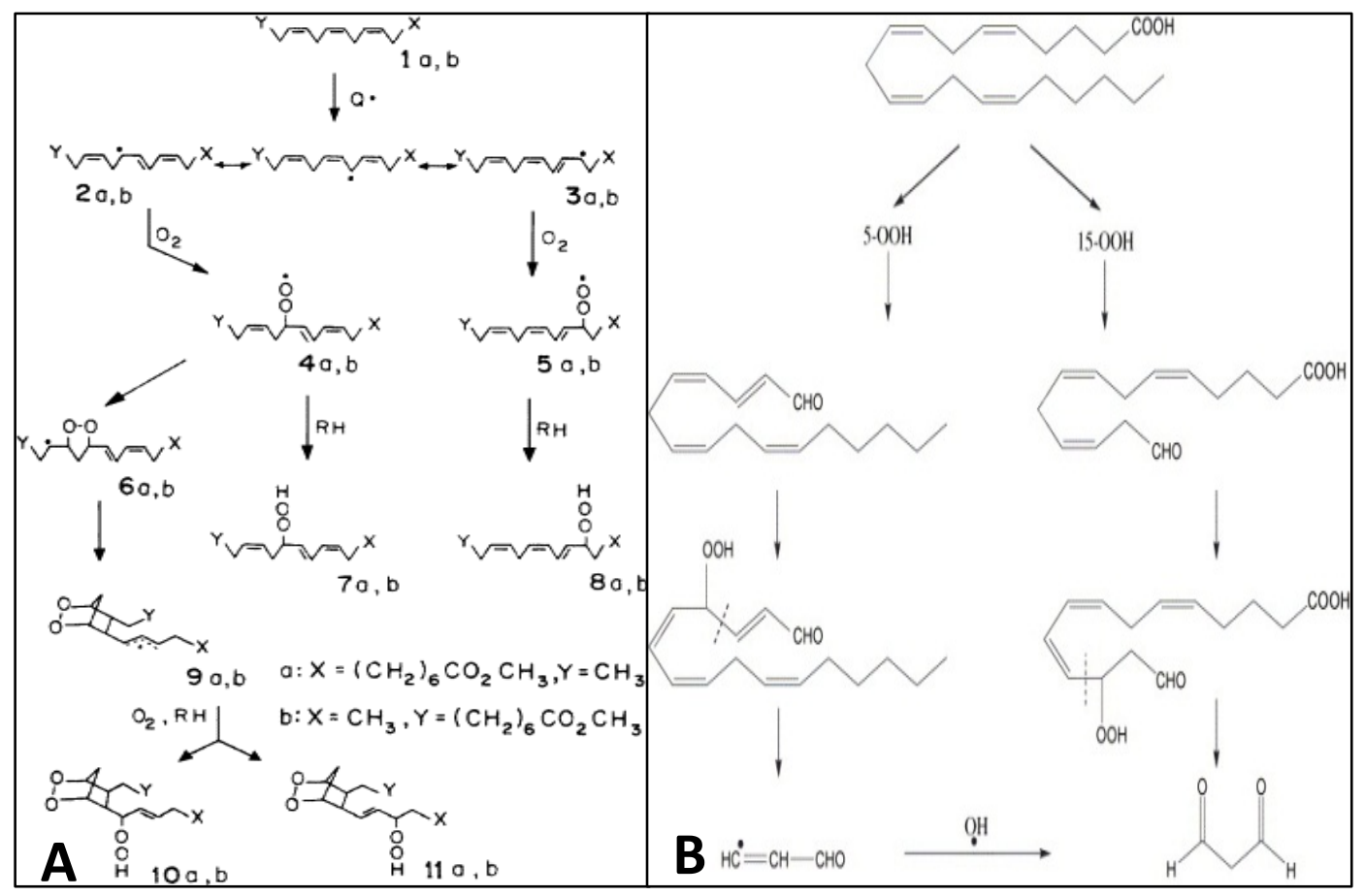

Figure 5. Two hypotheses of formation of MDA A) predicting that MDA is derived from prostaglandin-like endoperoxides from methyl-linoleate during times of stressful conditions and B) MDA is produced by beta-scission or by reaction of the final acrolein radical with a hydroxyl radical [13]. 
In vivo, MDA can appear in two different forms: free and total MDA. Free MDA is unbound to a protein found in plasma or any other biomolecule therefore it can be detected without a sample treatment requiring the sample to be chemically decomposed in water therefore providing a faster but less accurate method [13]. Total MDA is bound to molecules found in the matrix of the sample and requires a treatment of the sample in order to chemically separate the MDA from other molecules in order to be detected [13]. Examples of such treatments include methods using a strong acid or basic sample to pretreat in order to separate the bound fraction of MDA to obtain an estimate of the total MDA in the sample [13]. Little is known about the pathophysiological significance of either form however most methods used to detect MDA are for total MDA concentrations due to their high temperatures and acidic conditions during analysis [13]. There is a high percentage of variability between studies in baseline readings of total MDA levels in a healthy patient and in patients who have a TBI possibly due to the sample pretreatment used and/or method of detection. The relationship between TBI and systemic MDA levels are still being debated, as it is still a new area of research.

Another new area of research includes how levels of MDA vary with respect to time in patients who have TBIs. In a 2005 review article, 10 different studies were compared on their analysis of MDA in human plasma or serum in patients without any injuries [13]. The average MDA baseline concentration for 
healthy patients was $1.3795 \pm 0.153 \mu \mathrm{mol} / \mathrm{L}$ with an outlier of $13.8 \pm 1.32 \mu \mathrm{mol} / \mathrm{L}$ [13]. A German study in 2000 compared plasma MDA concentrations over time for isolated TBI patients and patients with other major trauma in addition to TBI [2]. The study found steady levels of MDA in surviving isolated TBI patients, a decrease in MDA levels for the first two hours following injury for the nonsurviving isolated TBI patients, and no relationship with patients with other major trauma (Figure 6A) [2]. A 2002 Italian study compared blood samples of TBI patients over 48 hours and compared the good and poor outcome group levels (Figures 6B and 6C) [6]. They concluded that MDA levels significantly increased over time in jugular bulb samples and the MDA levels were statistically significantly higher in the poor outcome group versus the good outcome group at 6 and 12 hours post-injury [6]. 

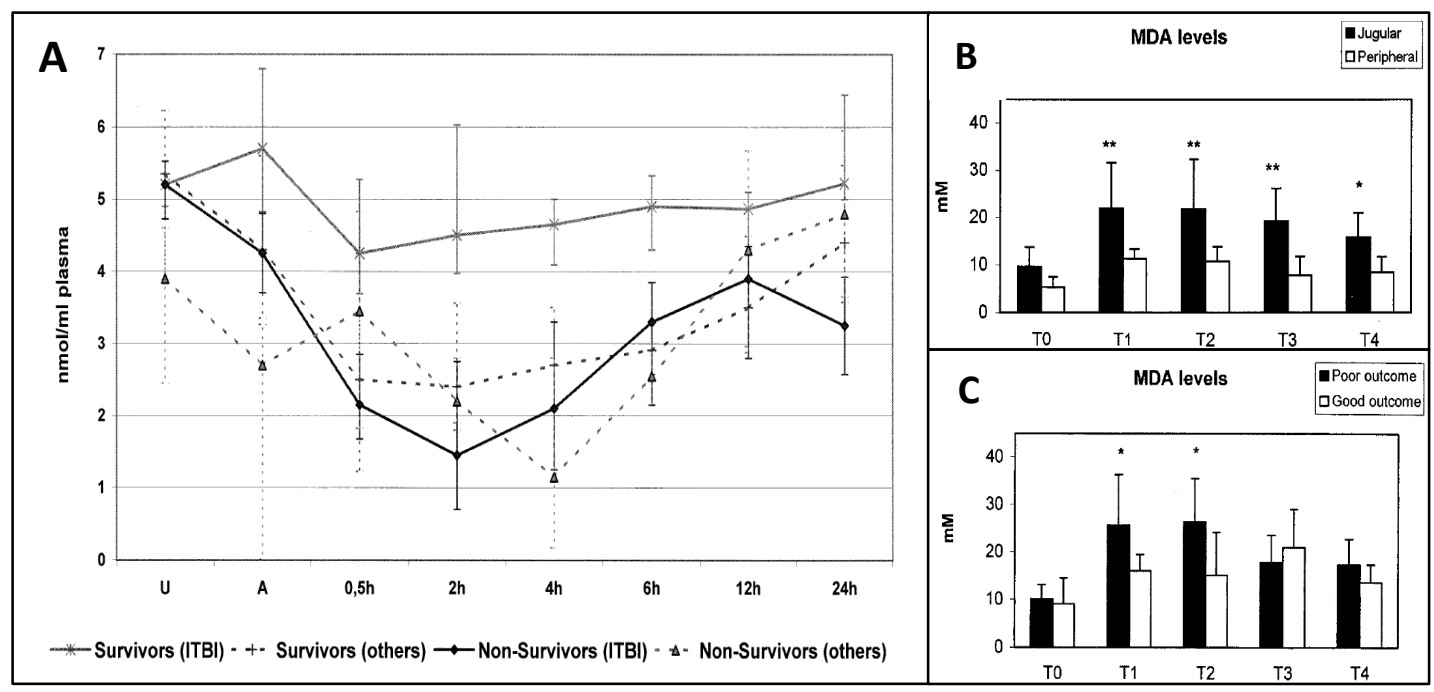

Figure 6. A) Relationship between time and MDA levels in patients with TBI [2]; B) MDA levels in jugular and peripheral blood over 48 hours [6], C) MDA levels in poor and good outcome groups over 48 hours [6].

One way to progress the affiliation between the two would require a standard test and a large population study to gain statistically significant results. Many researchers have begun attempting to decipher any correlation between systemic MDA concentrations, TBI outcomes, and time following injury. Current detection methods range from high power liquid chromatography to mass spectroscopy to fluorescent light detection [13]. One of the most common detection methods of total MDA is based on its derivatization with thiobarbituric acid (TBA) to form thiobarbituric acid-reactive substances (TBARSs) that fluoresce (Figure 7)[13,16,29]. The level of TBARS fluorescence can be correlated to specific concentrations of total MDA concentration in the sample. 

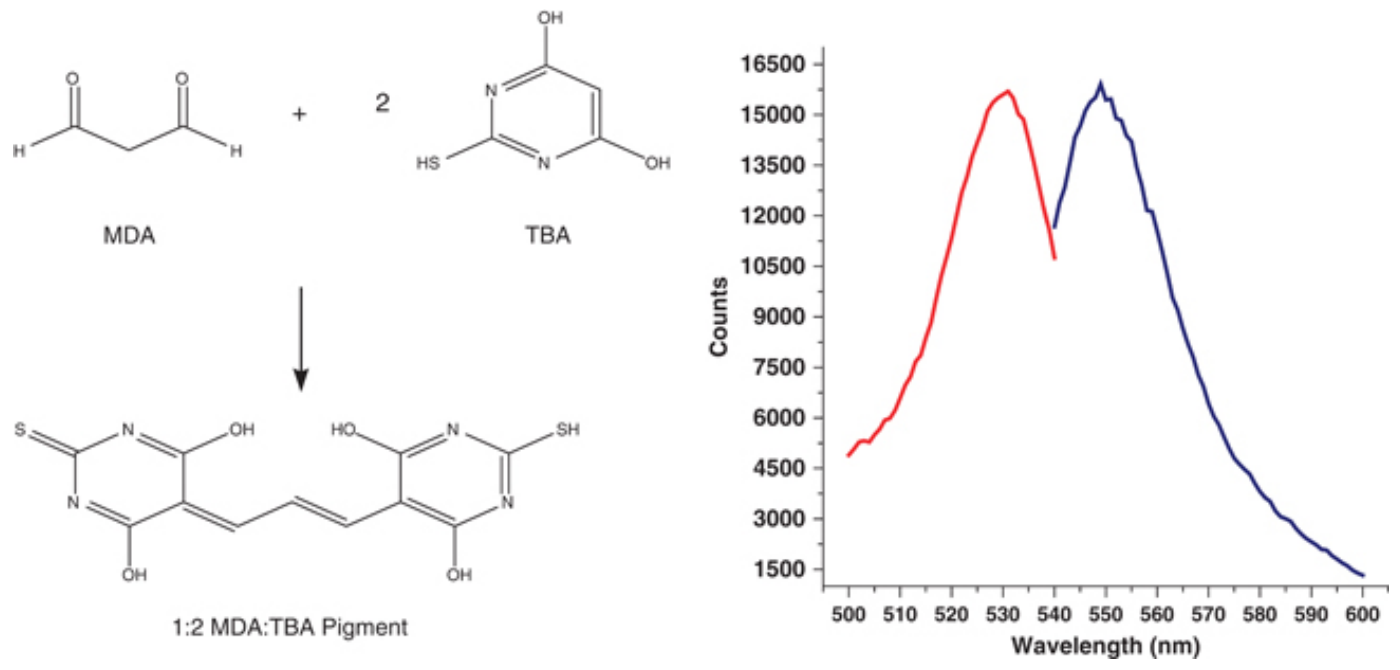

Figure 7. Reaction between MDA and TBA to form TBARS and the corresponding excitation and emission spectrums [29].

A large problem with the TBARS method is its lack of specificity within a sample as the TBA can bind to other molecules that may be present in the sample besides MDA to form TBARS [16]. New methods of detecting MDA in a sample, including capillary electrophoresis, have begun to be used and found to be very accurate with high precision [13].

Another method of detection includes high powered liquid chromatography (HPLC) with a solid-phase extraction technique in which the liquid sample is high pressure injected into a column filled with various sized particles [32]. The various particles get stuck along the tube in specific places forming separate bands in the column which can be used to separate, identify, and quantify compounds in a sample in concentrations as low as parts per trillion [32]. One large benefit of the HPLC method is it provides a high level of accuracy 
especially in samples with minimal concentrations of the substance and the columns can be repeatedly used [32]. However, HPLC requires equipment that cannot be scaled down and the equipment is extremely expensive [32]. Many researchers have decided these obstacles are difficult to overcome and are looking into developing miniaturized capillary electrophoresis (CE) systems.

Capillary Electrophoresis

Electrophoresis is defied as "the separation of charged species in a fluid when subjected to an electric field due to differences in the mobilities of individual species which in turn results in differences in the species velocity and therefore the distance travelled within a fixed time frame" [21]. In operation, induced electric fields, generally 50-250 volts/cm, mobilize ionic species to travel through a capillary based on its electrophoretic mobility and the strength of the electric field [21]. This technique is used for a variety of applications including the analysis of DNA, environmental and water quality metal ion detection, food and drink, and corrosion [21].

Electrophoresis is a type of fluid flow phenomenon in which a charged surface plus attached material move relative to stationary liquid by an applied electric field [23]. In order for this to occur, the system consists of buffer reservoirs, a sample reservoir, a high voltage power supply, electrodes, a capillary, and a detection system [22]. In the system, the capillary creates a double layer to allow for flow of the sample from the inlet to outlet [21]. Detection 
systems vary from laser-induced fluorescence to UV detection to electrochemical detection [21].

Electroosmosis is the movement of a liquid relative to a stationary charged surface, usually a capillary, by an applied electric field, such as in electrophoresis [23]. In order for this movement to occur, the electric double layer must be formed along the walls of the capillary. The electric double layer is an area next to the positively charged capillary surface in which ions, or positive and negative charged particles, attempt to neutralize the surface [23]. The ions are unable to offset the charge of the capillary surface due to the large number of negatively opposing ions compared to the positive ions created by the charge on the capillary (Figure 8 , top)[23]. The positively charged surface of the capillary wall causes the negative ions, or anions, in the fluid to be attracted and bind together in order to create an inner layer on called the stern layer (Figure 8, top) [21]. More cations, or positively charged ions, extend into the middle of the fluid of the capillary in order to attempt to offset the charge on the capillary surface and create the mobile layer [21]. The value of the difference in the charges of the ions between the mobile and stern layers is called the zeta potential [23]. The velocity of the fluid is dependent on the strength of the electric field applied to the capillary and the zeta potential [23]. The velocity provides a flat flow profile unlike the rounded profile found in laminar flow (Figure 8, bottom)[23]. By altering the $\mathrm{pH}$ of the solution, 
the velocity will change depending on its effect on the fluid in relation to the applied electric field [21].

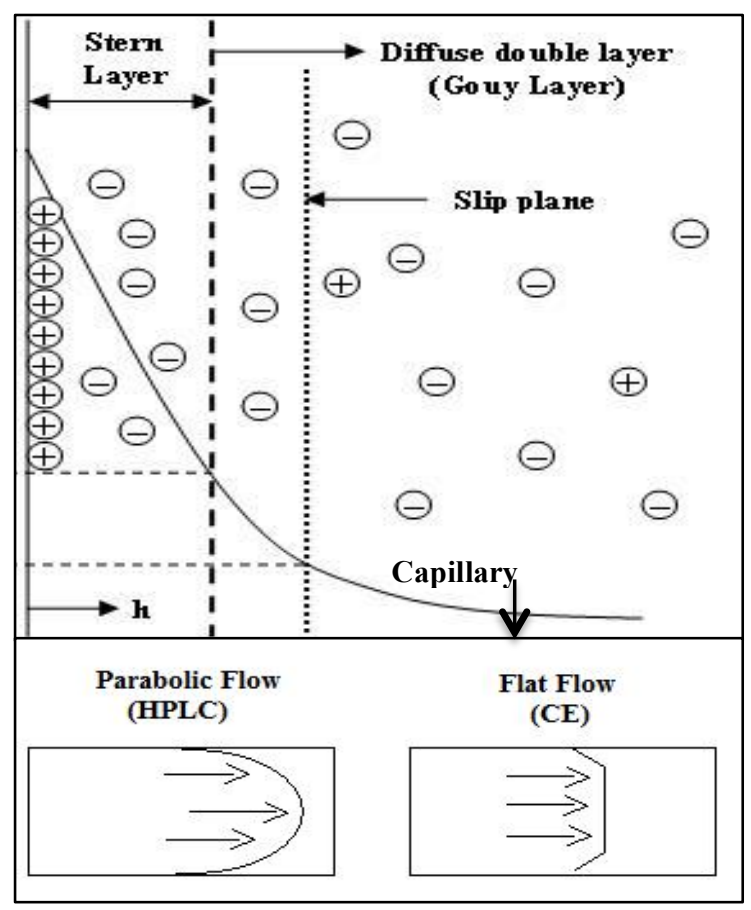

Figure 8. (Top) Schematic of the formation of the double layer [23]; (Bottom) comparison between parabolic flow caused by pressure differences and flat flow caused by capillary electrophoresis [23].

Electrophoresis comes in the form of gel and capillary methods where the capillary is either a gel slab or a capillary tube. In gel electrophoresis, the polyacrylamide or agrose gel acts as both the capillary and buffer [21]. The gel contains pores that allow particles to travel through it at various rates [21]. The sample is injected into the gel at one end and the positive electrode is placed at the other end [21]. When the voltage source is turned on, the voltage travels through the gel and causes the particles in the sample to flow towards the electrode at the 
other end [21]. The particle's velocity through the gel is dependent on the size ratio of pore to particle; so small particles can pass through the pores easier than that of a larger molecule so their velocity is greater [21]. This process requires a physical size difference between the charged molecules found in the sample, and can have a long separation time of hours or even days [21].

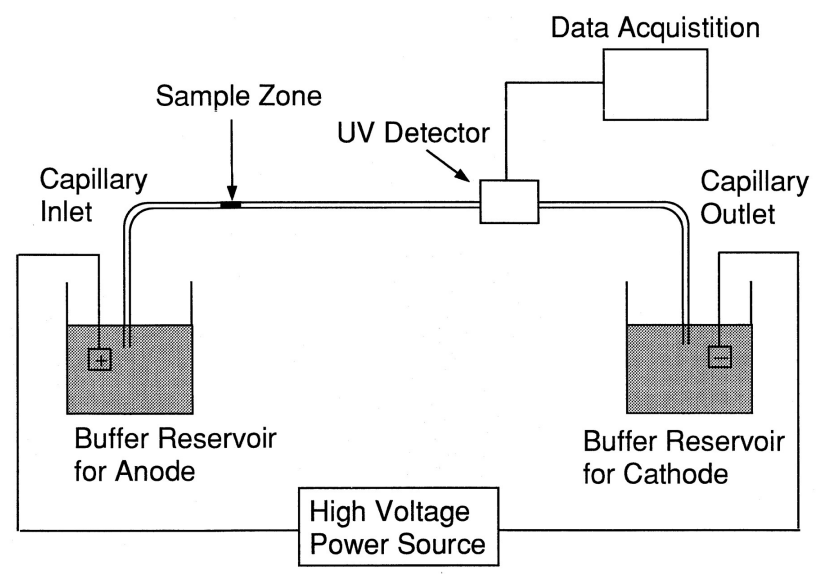

Figure 8. Basic schematic design of capillary electrophoresis system [21].

Capillary electrophoresis (CE) is comprised of two buffer reservoirs, positive and negative electrodes, a detector system, data acquisition system, and high voltage power source (Figure 9) [21]. The capillary is usually made of fused silica with varying inner diameters usually 25 to 100 microns [21]. The same principles for CE apply as with gel electrophoresis with the sample at one end, and the electrodes at either end [21]. When the voltage is applied, the particles found in the sample travel through the capillary at various rates based on their size and charge [21]. As the particles pass the detector window, the acquisition system 
records the type and concentration of particles as they pass by the window [21]. CE comes in multiple forms including capillary isoelectric focusing and isotachophoresis [21].

In capillary isoelectric focusing, usually proteins, are separated by adjusting the gradient of the $\mathrm{pH}$ of the buffer created in the capillary so the desired protein moves along the capillary until it reaches the $\mathrm{pH}$ where it is electrically neutral [21]. Isotachophoresis uses two different buffer solutions as a leading and terminating electrolyte with the sample falling between the two [21]. The different mobilities create different electric fields across the different sections and will not allow the sample to mix with the two buffers [21]. Unfortunately, the technique cannot separate samples containing both anions and cations in a single run as the mobilities oppose charges [21]. Currently, bidirectional systems are being developed to allow samples with both cations and ions to be separated at the same time in the system to reduce analysis time but this requires two separate detectors [21]. Each system was developed to detect substances in a variety of samples and is continuously being developed to increase efficiency for their intended purpose. 


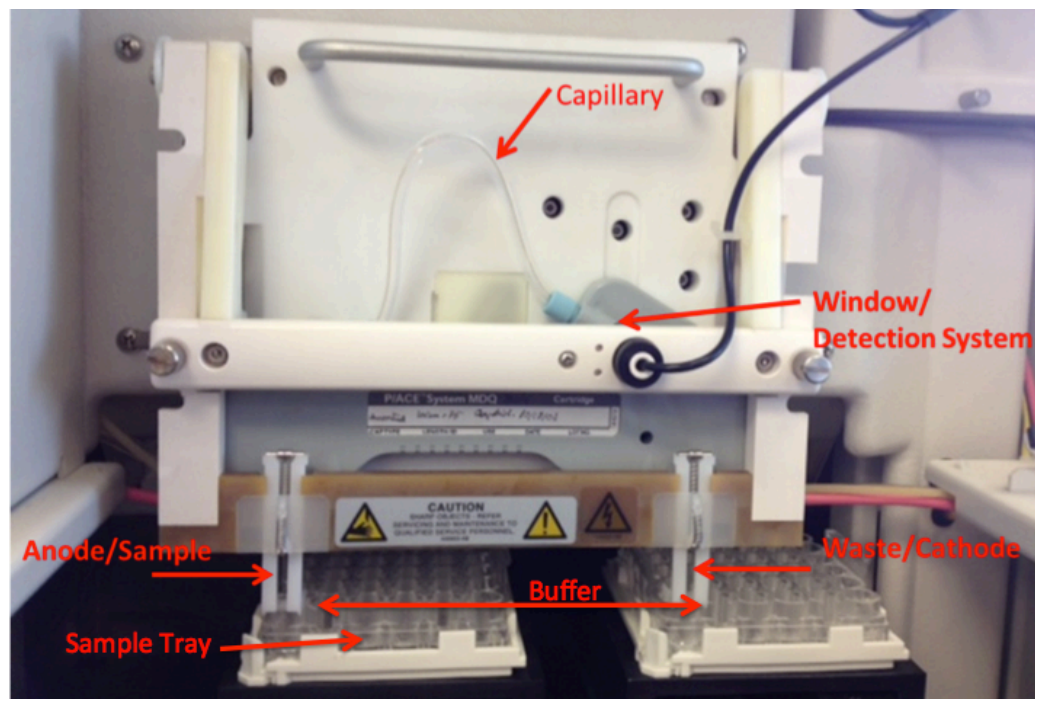

Figure 9. Table-top CE system used to detect charged molecules found in a sample (Cal Poly, Dairy Science Center).

Table-top CE systems are fairly common in standard laboratories as they provide an automated and fast analysis of a sample (Figure 10). Within the system, fused silica capillaries of various inner and outer diameters can be loaded into the system based on the sample type and desired outcome. A small window is usually etched into the capillary at a defined length away from the sample entrance to allow for detection, creating a capillary zone electrophoresis system. Multiple samples can be loaded into the system on the sample tray in various vials along with a known substance as the control. To begin the system, parameters, such as run voltage and time, are input into a directing computer that is attached to the machine. Next, the cleaning and run buffers are run through the capillary, usually by pressure injection, to prepare the capillary for the samples. The anode 
electrode is automatically lowered into the sample vial and a voltage is applied to draw the solution into the capillary (Figure 11).

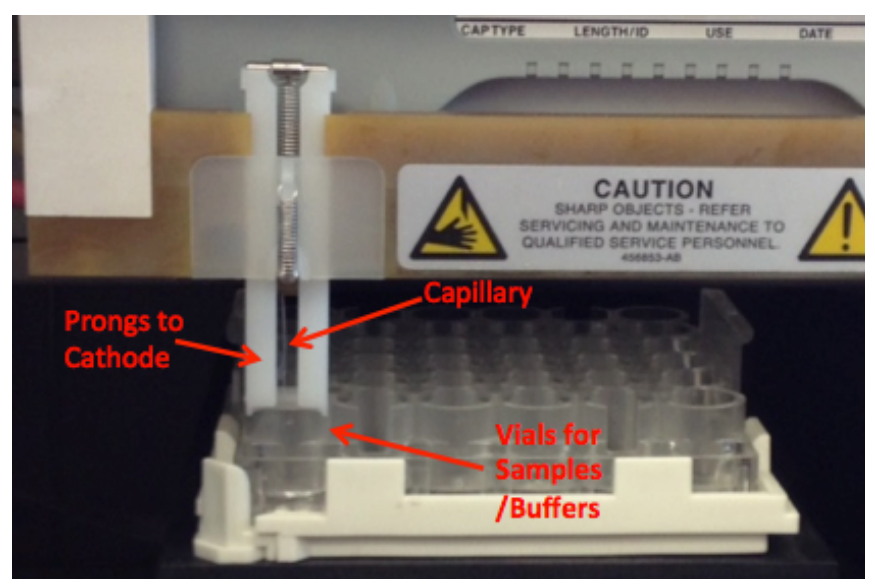

Figure 10. Sample end of table-top CE system with capillary and cathode electrode extending into vials of samples and buffers on the sample tray $(\mathrm{Cal}$ Poly, Dairy Science Center).

Between samples, the cleaning and run buffers are processed to cleanse the system of any remnants from prior samples. The capillary runs through the detection system that sends the data to the analysis computer system to be recorded. Finally, the capillary ends in the waste platform where the cathode is located and the sample waste is discarded (Figure 12). 


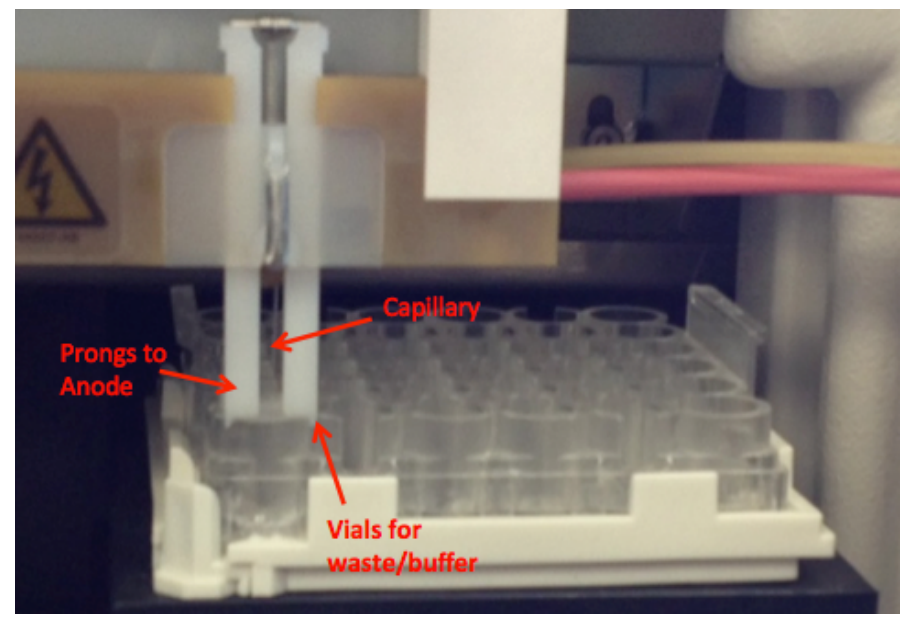

Figure 11. Waste platform of table-top CE system with capillary and anode electrode extending into vials for waste (Cal Poly, Dairy Science Center).

Currently, microchip CE systems are being developed as a form of a miniaturized system (Figure 13). Some benefits of a miniaturized capillary system include portable monitoring, reduced cost, disposability, reduction in required sample volume, faster analysis times, and the easier ability to generate a large electric field [21]. When designing a microchip CE system there are a variety of design considerations including the size, weight, power lifetime supply, device lifetime, self-directed (for sample loading, microfluidic priming and pumping, cleaning, and operation and analysis), universal buffering system with minimal to no sample preparation, and fast analysis performed on the microchip and displayed on the device [21]. Two of the most challenging aspects of developing a microchip CE system include the development of a sample injection system and the high voltage power supply. 


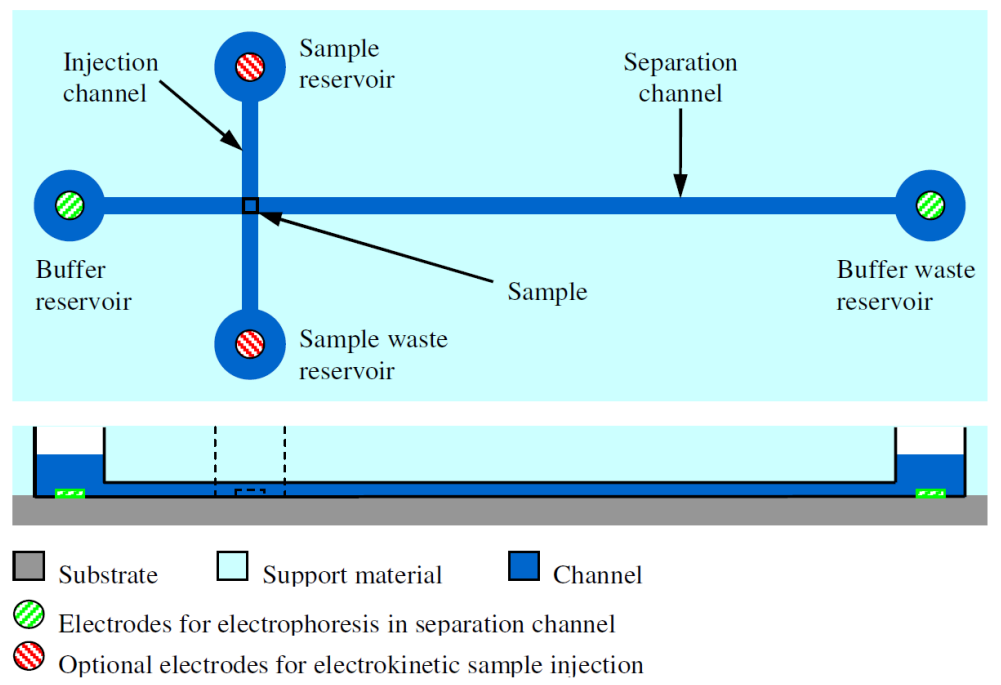

Figure 12. Typical layout for microchip CE device [21].

Sample injection systems are used to introduce the sample solution into the separation channel (Figure 14a) [21]. Some sample injection systems for a microchip design include electrokinetic injection and flow injection (FI). A FI system has the sample injected into the separation channel with a pressurized pump while the buffer fluid is concurrently flowing in the separation channel [21]. A form of FI uses a pinched injection scheme where the electric field between the buffer and waste reservoir causes fluid to flow toward the sample waste well where it intersects with the separation channel [21]. This allows for an accurate, repeatable, and highly efficient separation but requires a very precise fluidic control [21]. Electrokinetic injection uses an applied voltage to create an electric field between the sample and sample waste well to move the sample across the separation channel (Figure 14b) [21]. One the sample reaches the intersection, the electrodes in the buffer and waste reservoirs receive voltage and 
the plug travels down the separation channel (Figure 14c) [21]. This system does not require a mechanical system but can cause inaccuracies in readings or sample biasing due to variances in the mobility of the sample components. Different particles found in the sample leave the sample reservoir at various rates due to the applied electric field causing the sample to separate prior to reaching the separation channel once mixed with the buffer solution. Another problem that occurs during electrokinetic injection is caused by the applied electric field changing the $\mathrm{pH}$ of the solutions due to electrolysis that potentially can alter the behavior of the particle [21].

(a)

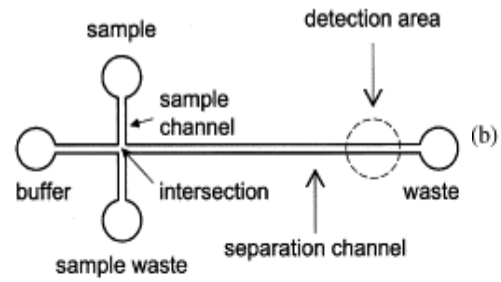

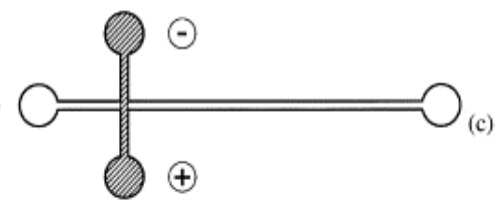

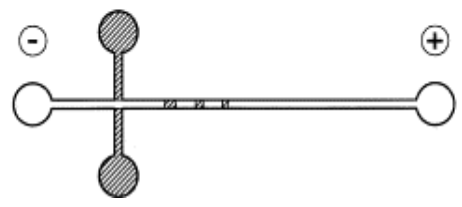

Figure 13. Setup and functionality of chip-based electrokinetic injection for CE system [21].

In order to create these electric fields, a high voltage power supply is required. When designing a high voltage power supply, there must be a compromise between size, power consumption, and performance [21]. Many protocols require voltages in the kilivolt range in order to move the charged particles in the sample for any distance in a short period of time [21]. However, using microchips allow for lower voltages due to significantly shorter capillary 
lengths. Still using high voltages require safety precautions to be designed into the system including the use of trip switches that turn off the voltage when the case is opened [21]. In order to produce high voltages for portable CE systems, researchers have optimized the process of using DC-to-DC converters that take a low voltage input and convert it to a much higher voltage [21]. Researchers are attempting to make chip-based and non-chip-based CE systems for portable applications.

Some chip-based CE systems include the $\mu$ ChemLab and a device created by Becker et al (Figure 13A and B)[21]. The $\mu$ ChemLab is a hand-held CE system is a battery powered, ergonomically designed device that weighs less than $2 \mathrm{~kg}$ and uses reusable microfluidic chips [21]. The $\mu$ ChemLab, Becker device, and a variety of others use their own high voltage power supplies. One system uses piezoelectric transformers due to their high transformation ratio and their smaller size and cost when compared to magnetic transformers [21]. The Becker system used two couple commercial miniature unipolar power supply modules to proved a bipolar driving power supply [21]. All of these devices were journal articles that were for detection of specific samples. Biomarkers, including ROS, are a large area of research with relation to $\mathrm{CE}$ as $\mathrm{CE}$ systems provide a strong platform for development of lab-on-a-chip systems to be used as point-of-care 
diagnosis.

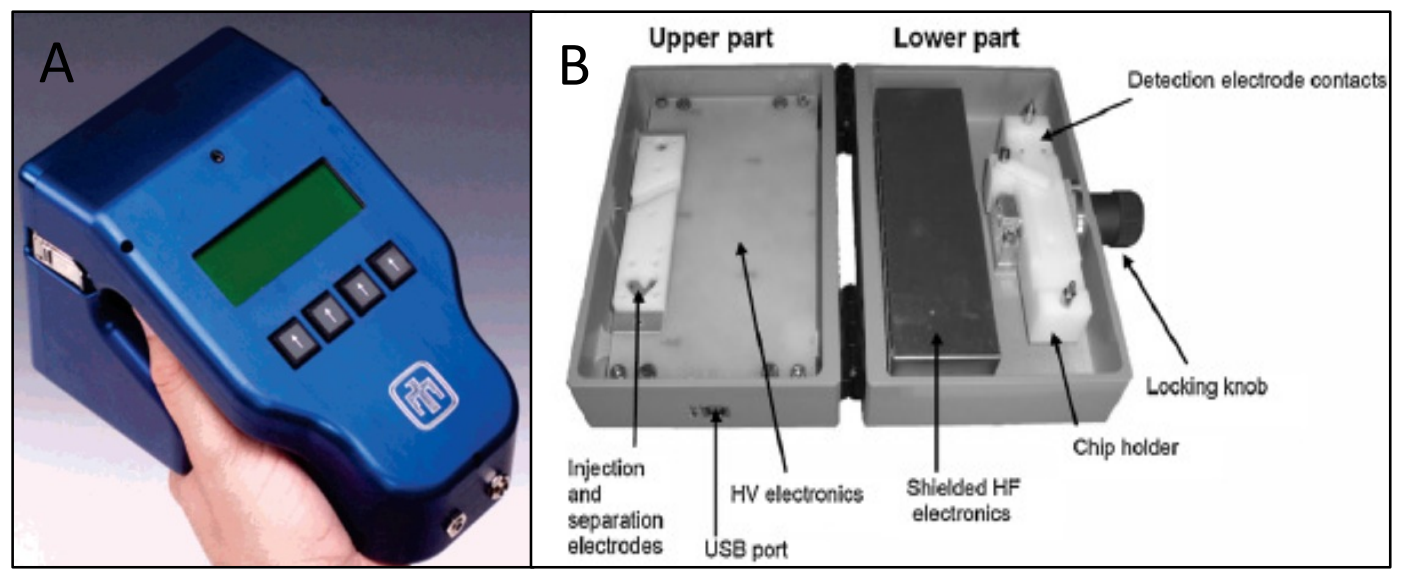

Figure 14. Examples of chip-based CE systems including (A) the $\mu$ ChemLab and (B) a system created by Becker et al [21].

Research into malondialdehyde detection in a sample using $\mathrm{CE}$ is becoming very popular as many labs have the systems. It can have fairly precise and repeatable readings, and it has a short analysis time [21]. Biological samples for MDA detection include urine, blood, and breath condensate however the most common is human plasma from whole blood [13]. Most articles use plasma from healthy individuals, then add MDA salt to the sample and compare the detected concentration to the input concentration to determine the accuracy of the process [13]. A large benefit of microchip CE systems is their ability to use a minor volume of blood sample to produce an accurate representation of concentration in the body [21]. Many have found that CE, especially high performance CE, is more accurate than the traditional method of fluorimetry [21]. Various parameters are used for detection of human plasma MDA including the inner diameter of the 
capillary, total and run lengths of the capillary, the run and cleaning buffer solutions, and the run voltage (Table 1) [24, 25, 26, 27]. The variation of parameters is due to the various models of table top CE machines and preferences of the difference laboratories. In addition, the process of detecting MDA with $\mathrm{CE}$ systems is still being perfected so multiple methods are attempted in order to determine the most accurate and repeatable process. The conclusion for all of the articles was the high accuracy of detection of free MDA in the sample and the strong potential to create a microchip based CE system for point-of-care diagnostics for the detection of MDA in oxidative stress situations [24, 25, 26, 27].

Table 1. Parameters used in various studies using CE to detect MDA in human plasma $[24,25,26,27]$.

\begin{tabular}{|c|c|c|c|c|c|}
\hline Article & ID of tubing & Lngth of tube & run buffer & run voltage & Cleaning buffer \\
\hline R. Paroni [24] & 50 micron & $\begin{array}{l}27 \mathrm{~cm} \text { total } \\
20 \mathrm{~cm} \text { run }\end{array}$ & $\begin{array}{l}25 \mathrm{mmol} / \mathrm{L} \text { borax } \\
5 \mathrm{mmol} / \mathrm{L} \text { TDTAB } \\
\mathrm{pH} 9.3\end{array}$ & $-4 \mathrm{kV}$ & $\begin{array}{l}1 \mathrm{~mol} / \mathrm{L} \mathrm{NaOH} \\
0.1 \mathrm{~mol} / \mathrm{L} \mathrm{NaOH}\end{array}$ \\
\hline A. Zinellu [25] & 75 micron & $\begin{array}{l}60 \mathrm{~cm} \text { total } \\
50 \mathrm{~cm} \text { run }\end{array}$ & $\begin{array}{l}300 \mathrm{mmol} / \mathrm{L} \text { sodium borate } \\
50 \mathrm{mmol} / \mathrm{L} \mathrm{N} \text {-methylglucamine } \\
\mathrm{pH} 10.0\end{array}$ & $25 \mathrm{kV}$ & $0.5 \mathrm{mmol} / \mathrm{L} \mathrm{NaOH}$ \\
\hline D. Wilson [26] & 75 micron & $60 \mathrm{~cm}$ total & $\begin{array}{l}10 \mathrm{mmol} / \mathrm{L} \text { sodium tetraborate, } \\
10 \mathrm{mmol} / \mathrm{L} \text { sodium monosphate, } \\
100 \mathrm{~mL} / \mathrm{L} \text { acetonitrile } \\
\mathrm{pH} 8.0\end{array}$ & $18 \mathrm{kV}$ & \\
\hline K.N. Korizis [27] & 50 micron & $56 \mathrm{~cm}$ active & $\begin{array}{l}20 \mathrm{mM} \text { borate } \\
\mathrm{pH} 9.3\end{array}$ & $30 \mathrm{kV}$ & $\begin{array}{l}.01 \mathrm{M} \mathrm{NaOH} \\
\text { DI Water } \\
\text { Run Buffer }\end{array}$ \\
\hline
\end{tabular}

All of the articles that discuss plasma MDA concentrations and its relation to TBI, state the lack of information available, and the strong potentials that the information could provide. As the relationship between MDA and TBI is slowly 
being understood, further research is required in order to fill the lack of information in the field of study. A standard method of detection could prove invaluable as the concentrations of MDA vary pending on the various techniques and variables between the systems. One common and accurate sample types used to detect MDA is blood. Unfortunately there are many items found in blood that can give false readings and can make detection impossible. In order to get the most accurate reading, plasma containing the MDA needs to be separated out of the sample.

Blood Filtration

There are multiple types of biomarkers that can be found in the body via a variety of biological samples including biopsied tissue, breath condensate, urine, saliva, and blood. Blood is frequently used as a biological sample when looking for specific biomarkers due to its ease to obtain and its high concentrations of certain systemic and circulating molecules such as proteins. MDA is found in a variety of biological samples but most researchers use blood due to its ease of access unlike more dangerous methods of sample acquirement such as cerebral fluid [9]. Additionally, MDA can enter the blood stream via the blood-brain barrier and is quickly circulated throughout the body. One large challenge of using blood as a biological sample for biomarkers is the required pretreatment methods in order to separate out artifacts that could give inaccurate results. 
Whole blood is comprised of platelets, white blood cells, red blood cells, and plasma each ranging in size (Table 2). White blood cells (WBCs), or leukocytes, come in a variety of shapes and sizes consisting of neutrophils, monocytes, macrophages, and lymphocytes which are responsible for the immune system in the body [17]. Platelets or thrombocytes are irregularly shaped fragments of cells that circulate in the blood to be activated in the formation of a blood clot [17]. Red blood cells, or erythrocytes, are the most common type of cell in blood, most dense substance in blood, and have a biconcave shape to allow for a greater surface area to transport oxygen throughout the body [17]. Plasma is the straw colored fluid comprising approximately $60 \%$ of blood consisting mainly of water with some proteins, antibodies, hormones, sugars, and fat particles [17]. When looking for biomarkers, most bioseparation tests require plasma therefore the other components must be separated from whole blood prior to the test.

Table 2. Sizes of components found in whole blood [17].

\begin{tabular}{|c|c|}
\hline Blood Component & Diameter (microns) \\
\hline White Blood Cells & $7-15$ \\
\hline Platelets & $2-4$ \\
\hline Red Blood Cells & $6-8$ \\
\hline
\end{tabular}


In order to stabilize the blood during the withdraw process, additives such as anticoagulants are used as pretreatment techniques to help separate blood components from the plasma [18]. However, some of these anticoagulants can have a strong influence on results from the sample [13]. When determining MDA concentrations in the body, researchers have determined that different anticoagulants have a strong influence on plasma MDA concentrations [13]. For example, plasma treated with EDTA showed lower concentrations when compared to citrate treated samples due to the fact that citrate does not bind to trace metals or calcium ions in the blood therefore preventing more MDA production after the blood has been drawn [13]. This observation that the use of anticoagulants during pretreatment could explain the variances in MDA values [13].

When preparing the sample, a variety of filtration methods have been used including centrifugation, membrane filters, and microfluidic chips. Centrifugation is the most common method for separating whole blood into plasma however it cannot be utilized for point-of-care diagnostic devices. Membrane filters such as the Vivid ${ }^{\mathrm{TM}}$ and Accuwik ${ }^{\mathrm{TM}}$ Plasma Separation Membranes have been developed to replace centrifugation to obtain plasma for biological testing [19]. These membranes have an asymmetric shape that catches cells in large pores on one side and allows the plasma to pass through the smaller pores to produce a sample comparable to that of one that has processed through centrifugation [19]. 
Recently microfluidic chips have been designed and optimized with the potential to be integrated with another chip-based bioseparation analysis technique [20]. Electrical fields have been shown to separate particles and cells out of solutions, which makes it an excellent method to apply to chip-based technologies. In a study by Jiang and associates, electric fields were applied to move cells and particles in various directions based on size and strength of the applied electric field [20]. Electro-osmotic flow moves items with weaker electrostatic charge such as liquids [20]. Electrophoresis of cells and particles is due to electroosmosis movement from stronger electric fields [20]. The main channel on the chip transports the bulk solution with a strong electric field while weaker electric fields move across the branching channels to separate the liquid out into the branches [20]. Branching channels are narrower than the main channel that prevents cells and particles from entering [20]. Additionally, the electro-osmotic flow that is created by the field moves the plasma from the bulk into the branching channels [20]. 


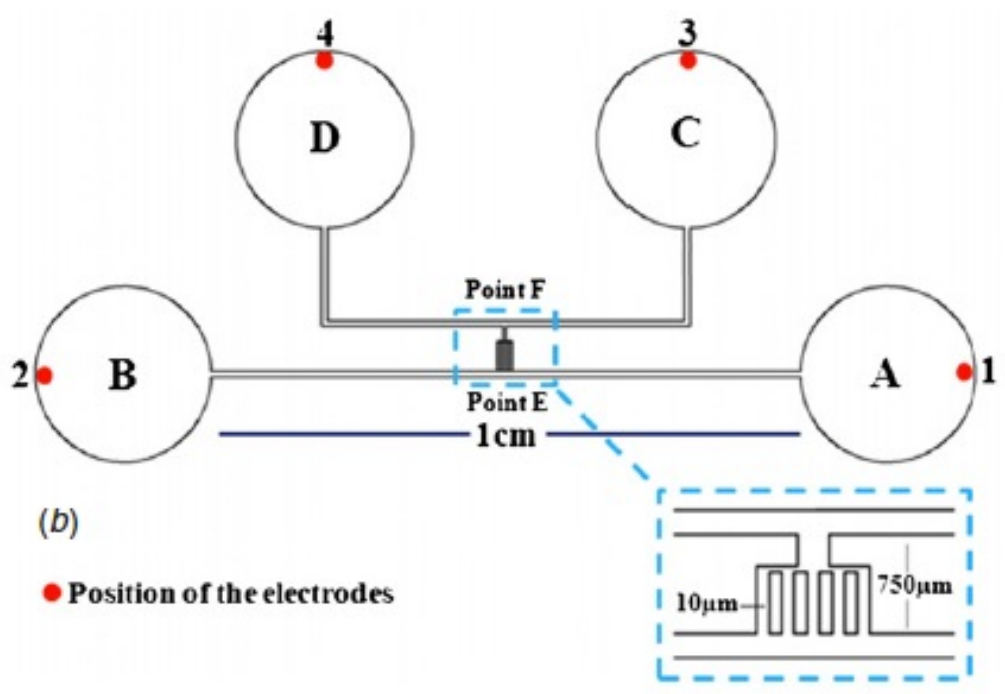

Figure 15. Diagram of an example microchip design used to separate whole blood in Jiang experiment [20].

The chip from the Jiang experiment consists of four wells, a main micro channel, five branching channels, an assistant channel, a neck channel, and four electrodes (Figure 15) [20]. The wells or reservoirs were filled with the buffer solution and well A also contained the sample [20]. The main micro channel ran between reservoirs $\mathrm{A}$ and $\mathrm{B}$ and provided the location for movement of the bulk solution [20]. The assistant channel runs between wells C and D and moves separated plasma from the neck channel to well D [20]. The five branches between the main and assistant channels are the location for the separation of plasma out of the bulk solution [20]. The neck channel connects the branch channels and the assistant channel [20]. Finally, the four platinum electrodes are placed in each well and control the applied electric field by supplying voltage to the system [20]. 
Fabrication of the chips utilized a soft lithography process for creating the master chip and molding for the test chips [20]. The master process consisted of spin coating a negative photoresist onto a silicon wafer that was then pre-baked [20]. Next, the wafer was exposed to a mask pattern using UV light and then postbaked [20]. Finally, the chip was developed with the locations of the various channels [20]. Test chips were then made with a degased polydimethylsiloxane (PDMS) solution combined with a curing agent [20]. Once cured, the chips were peeled off the master wafer, holes for reservoirs were then punched out, and the chip was bonded with a glass slide to create the channels [20].

At the conclusion of the study, it was determined that the system was successful in providing a proof-of-concept device that provided a method for separating blood and plasma using electro-osmotic forces [20]. The developed method was able to keep almost $100 \%$ of the cells out of the branch channels by optimizing the voltages leading to a controlled applied electric field to move the cells and liquid [20]. The benefits of this system include its lack of requirement for complex microfabrication techniques and absence of external pumping devices [20]. It is very possible to use this technology and process in a point-ofcare diagnostic device in the near future. Other future work includes more research into using the separated plasma for further analysis. The next step will be to develop other testing processes on a chip that can be integrated with this procedure. Early detection of MDA in a point-of-care diagnostic CE microchip 
device may help to provide a better level of care for patients and to help develop techniques and pharmaceudicals to reduce oxidative stress damage to neural tissues. 


\section{Design Process}

Introduction

The goal of this thesis project was to design a proof-of-concept field system in order to detect reactive oxygen species in the body that are released following a traumatic brain injury. In order to meet the goals of the device, the following general requirements were determined for the final device: fueled by own energy source (cannot plug into electrical outlet), cannot rely on any other power-operated machines (no centrifuge machine), and suitable in size and durability to be carried with a field medic. These design requirements were vague as research determined from this proof-of-concept would help develop more defined requirements for later prototypes. In order to accomplish this task, the device was divided into three main sections: blood separation, sample separation, and detection. This thesis report will examine the blood and sample separation sections of the device. The following section discusses the design process used for determining the blood separation technique and developing the proof-of-concept sample separation device.

Designs

\section{Blood Separation}

The first portion of the system requires whole blood to be separated into plasma and other constituents prior to being injected into the device. The blood filtration portion must meet the same requirements as the entire device, and must 
be compatible with the sample separation portion of the device. The three options considered included centrifugation, membrane filters, and a microfluidic chip.

The separation technique would need to be able to be implemented into a point-of-care diagnostic device, therefore traditional centrifugation techniques would not be appropriate for the application as they require electricity to run the preexisting machines. The membrane filters, such as the $\mathrm{Vivid}^{\mathrm{TM}}$ and Accuwik ${ }^{\mathrm{TM}}$ Plasma separation membranes, previously discussed in the blood separation section, are better suited to the field aspect of the requirements. Unfortunately, the membrane comes in sheets that would require building an additional contraption to hold the membrane. The additional blood filtration unit would be used to filter the blood after being drawn from the patient. The plasma that would be separated out would then be injected into the sample separation designs.

Recreating the microfluidic chip study (discussed in the previous section) would be ideal for this application as it could be integrated into a complete system. The blood could be injected into the blood separation design, where it would separate into plasma and then flow into the CE separation system for analysis. Essentially, the system would require the user to inject whole blood into the blood separation channel and the system would then separate and analyze the MDA from the plasma.

California Polytechnic State University, San Luis Obispo requires any data collected from tissue samples from human subjects must have the approval of 
Human Subjects Committee (HSC) prior to data collection. The review of the application for the desired study of the proposed protocol could take anywhere from 3 weeks to over 2 months. Due to the confidentiality and timeline of the project, it was not feasible to perform these tests.

\section{Sample Separation}

\section{Initial Designs}

The next aspect of the device considered was the sample separation design. The same design aspects were considered when selecting the final design of the sample separation. Three techniques were considered when selecting the design to continue forward with developing: fluorescent dyes, liquid chromatography, and capillary electrophoresis.

Fluorescent dyes can be used to identify particles in a sample by multiple methods including binding to the desired subject and emitting fluorescent light [37]. Advantages of this method include the ability to detect low abundant and sub-resolution molecules and it is well understood [37]. Disadvantages of fluorescence include its complexity of the procedure as it can require long incubation times with specific high temperatures in order for the dyes to correctly initiate [37]. In addition, fluorescent dyes used to detect MDA in samples can produce artifacts, or inaccurate anomalies, that can provide false results [37]. 
High performance liquid chromatography (HPLC) detects particles and their concentrations by running the sample, dissolved in a known solution, through a tube with stationary particles to inhibit the flow of some particles and allow others including the desired specimen to pass through to be detected. The advantages of HPLC include high accuracy of detection and short separation times (5-25 minutes) [32]. Shortcomings of HPLC include its high cost of the columns, between $\$ 475$ and $\$ 750$ based on the desired size [33]. In addition, feasibility of miniaturizing a HPLC system was determined to be difficult as published work on the feat was lacking when compared to information found on shrinking a capillary electrophoresis (CE) systems.

CE systems use high voltages to separate charged particles in medium in order to separate and detect concentrations of desired items in a sample. Some advantages of $\mathrm{CE}$ include its fast separation time, minimum requires sample volume, and significant amount of available information especially with regard to miniaturization and detection of MDA [21]. Some disadvantages of CE include its high voltage requirement and the dangers associated with using high voltages.

After comparing the three systems with regard to the positive and negative arguments, it was determined that capillary electrophoresis was the best option to meet the requirements specified by the sponsor especially due to its feasibility. 
After viewing the table-top CE system from Cal Poly's Dairy Science Center, multiple designs for CE systems were brainstormed.

One design for the $\mathrm{CE}$ system was a single use cartridge (Figure 16) that would be inserted into a reusable CE system. The cartridge would contain a specific volume of buffer that would mix with the sample that would be injected from a syringe. The system would consist of electrodes, a vacuum, and a detection system that could be reused as the samples would never make direct contact or cause contamination of any parts. However, the cost of replacing the fused silica capillary would not be cost effective as they cost approximately $\$ 10$ per meter and the lengths necessary could be too long for a field device [34].

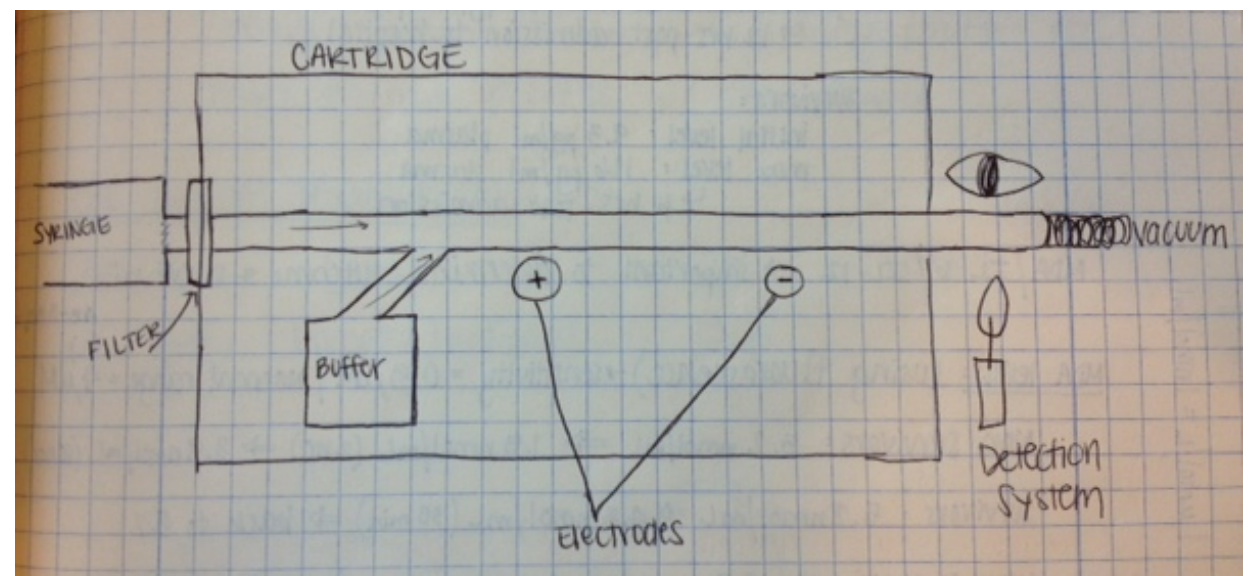

Figure 16. Sketch of a brainstormed idea of a CE system using single-use cartridges. 
Another CE system concept that was considered was a basic miniaturized system. The fused silica capillary would be cleaned between samples and therefore could be used for a maximum of 400 runs if properly cleaned and stored [31]. One system consisted of a series of tanks and valves in which the plasma is separated from the blood then released into the mixing tank via a valve where it mixes with the buffer solution (Figure 17). The vacuum would pull the sample into the capillary where the voltage would be applied to the electrodes and move the desired MDA down the capillary toward the window and UV detection system, and finally ending in the waste tank (Figure 17).

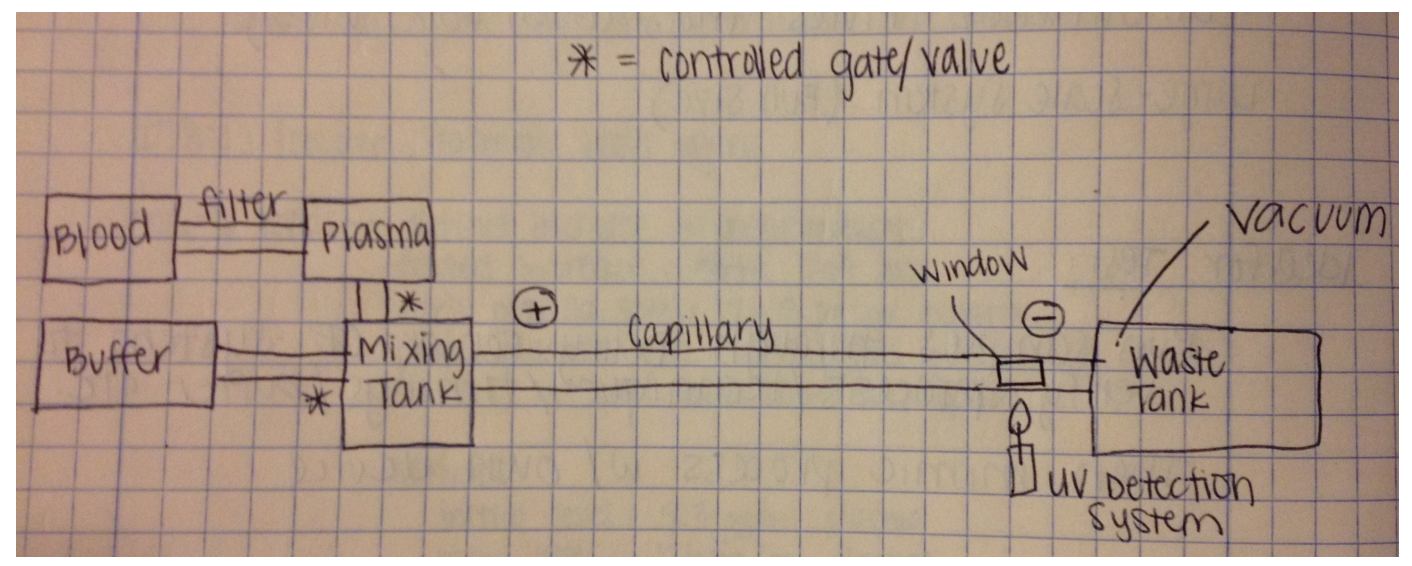

Figure 17. Drawing of a brainstorm of a simplified CE system design consisting of various valves and tanks.

Another simple CE model consisted of two wells, a capillary, electrodes, vacuum, detection system, and power source contained in a simple housing container was designed as a bench top model (Figure 18). The sample would be 
filtered and combined with the buffer externally, then pipetted into the cuvette on the left in the figure (Figure 18). Next, the vacuum would pull the sample into the capillary where voltage would be applied to the electrodes and the MDA would flow to the detector and finally end in the second cuvette as waste.

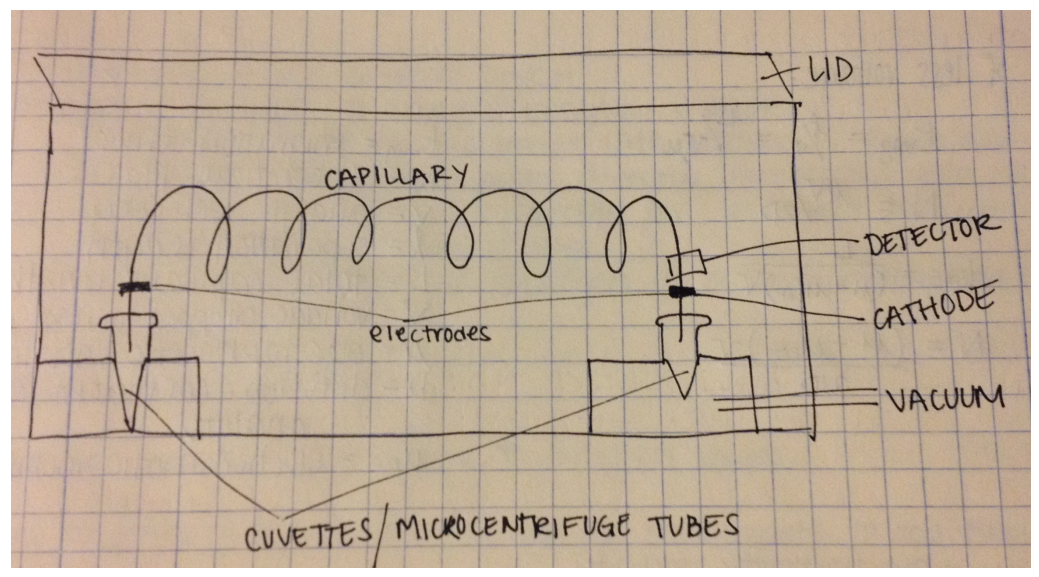

Figure 18. Drawing of a brainstorm of a simplified CE system design with an external blood separation system housed in a simple housing container.

After comparing the various brainstormed ideas, a basic, scaled-down CE system was then determined to be the best method to proceed forward as it met all the design requirements and appeared to be the most feasible. While looking for supplies and reading articles to learn more about miniaturizing CE systems, purchasing a pre-fabricated microfluidic chip was considered. The chip could provide wells for the sample and buffer solutions, various shaped capillaries, and it is all self-contained therefore it would fulfill the need for a housing container, wells, and capillary. As the capillary length could be significantly shorter than typical tabletop systems, voltage could be decreased and separation times would 
still be in minutes with the ability to view the moving solution at any portion along the capillary by using a microscope. In addition, the chip can be cleaned after each run and could be used for a maximum of 400 runs if properly cleaned and stored [21].

Microfluidic chips can be fabricated with any desired design however, more complex designs cause the price to increase if it is not an off the shelf layout. Due to the particles moving based on their charge attraction down the capillary, chip design was considered based on cost and simple geometry in order to make cleaning the capillary easier. After company research, the cross-channel layout was determined to be the most cost-effective and met the needs for a $\mathrm{CE}$ system.

The chosen and purchased chips were three pre-fabricated microfluidic chips of 2 inches long by 1 inch wide [35], which had a cross-channel design with four reservoirs (buffer, sample, buffer waste, and sample waste), an injection channel, and a separation channel (Figure 19) [35]. The channel dimensions were $100 \mu \mathrm{m}$ wide by $100 \mu \mathrm{m}$ deep, and the separation channel was $28.0 \mathrm{~mm}$ long [35]. The dimensions for the rest of the chip were two $7.0 \mathrm{~mm}$ long sample channels, the $10.0 \mathrm{~mm}$ long sample channel, the $1.0 \mathrm{~mm}$ diameter access holes, and the thickness of the top and bottom of the chip, which varies, based on orientation of the chip (Figure 19) [35]. 


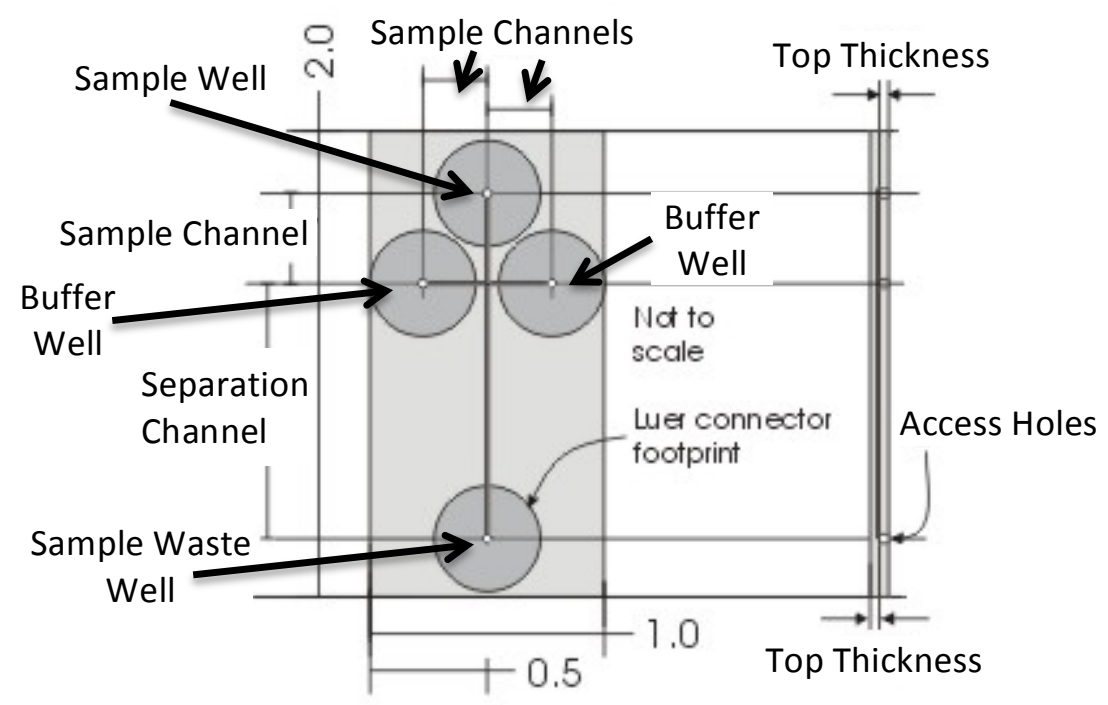

\section{$\underset{\text { Diawng: }}{\text { Copyright Translume }}$ \\ Pev 2}

Figure 19. Drawing of the pre-fabricated microfluidic chips purchased from Translume [35].

Two of the chips were bottom-up orientation, meaning the bottom of the chip is thinner than the top to use on an inverted microscope, with a base of 0.17 mm height, that was used for the confocal microscope [35]. The last chip was a top-down orientation, with a base height of $1.0 \mathrm{~mm}$, that was used for table-top microscope applications [35]. On the top of the chip, female luer lock connectors were mounted to connect with male luer lock connectors and to make sample placement into the wells easier (Figure 20). Loose compatible barbed male luer connectors were also purchased from the same company for attachment of other supplies to the microchip. Table 3 lists all products from Translume and their associated costs. 


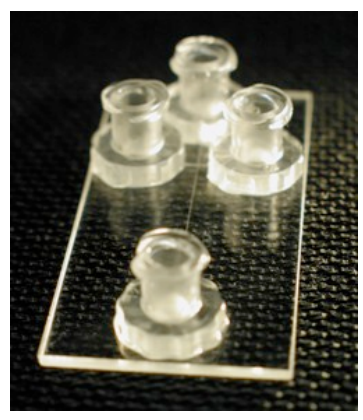

Figure 20. Chip with attached female luer lock connectors [35].

Table 3. Cost of Products from Translume [35].

\begin{tabular}{|l|l|l|}
\hline Item & Quantity & Unit Price \\
\hline Bottom-Up Chip & 2 & $\$ 202$ \\
\hline Top-Down Chip & 1 & $\$ 302$ \\
\hline Luer Connectors & 2 & $\$ 25$ \\
\hline & Total Cost & $\$ 756$ \\
\hline
\end{tabular}

\section{Final Design of Proof of Concept}

The final proof-of-concept design consisted of the separation portion of the system. The prototype consists of a microfluidic chip, two luer connector caps, two male-female luer lock connectors, two female-female connectors, two female T-shaped luer connectors, two bare wired electrodes attached to two male luer connectors, and a luer connector syringe (Figure 21 and Table 4). 


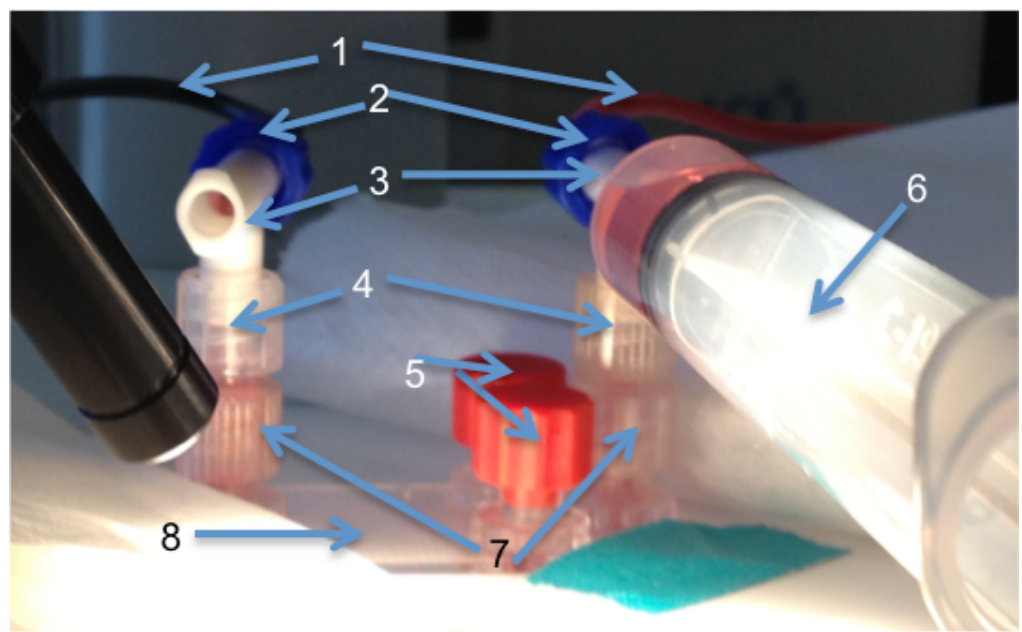

Figure 21. Prototype with main components labeled.

Table 4. Table of labeled items found on Figure 21 of prototype.

\begin{tabular}{|c|c|}
\hline Number on Image & Component \\
\hline 1 & Bare Wired Electrodes \\
\hline 2 & Male Luer Connectors \\
\hline 3 & T-Shaped Luer Connectors \\
\hline 4 & Female-Female Connectors \\
\hline 5 & Luer Connector Caps \\
\hline 6 & Luer Connector Syringe \\
\hline 7 & Male-Female Luer Lock Connectors \\
\hline 8 & Microfluidic Chip \\
\hline
\end{tabular}

To build the device, two wells on the microfluidic chip were capped in order to only allow flow through the separation and sample channel. The two capped wells are provided for later development of the device. Next, the malefemale luer lock connectors were attached to the luer connectors found on the 
sample and waste wells of the chip. Then the female-female connectors were attached to the chip on one end and the t-shaped connector on the other. Finally, the t-connector had one end had the bare wire electrode heat wrap attached with a luer connector, and the sample end had a filled syringe while the waste end was open to the air (Figure 21). 
Methods and Results

The following section discusses the experiments that were performed in order to demonstrate the feasibility of the capillary electrophoresis microfluidic chip to detect malondildehyde which has been linked to traumatic brain injuries. The series of experiments was designed to validate portions of the system in order to pinpoint any potential problems that could arise. First, the chip was tested with pressure driven flow of microspheres in order to confirm accurate fluid flow through the $100 \mu \mathrm{m}$ wide by $100 \mu \mathrm{m}$ deep channels. Next, the chip was tested with pressure driven flow of MDA solution in order to verify that it would flow through the channels and pressure driven flow could be used if voltage driven flow failed. Then, voltage was applied to the microsphere solution in a chip in order to learn how to set up a CE system. Finally, the MDA was used in place of the microsphere solution in order to validate the proof-of-concept for a potential field device to determine if it is feasible to continue.

Pressure Driven Flow on Confocal Microscope

Microspheres

The first test flowed fluorescent microspheres through the microfluidic chip to ensure proper flow and to practice the microchip set up on a confocal microscope. Two barbed male luer connectors were attached to the buffer and buffer waste reservoirs, and 1/8" inner diameter tubing was connected to the barbed end of both of the male luer connectors (Figure 22). 


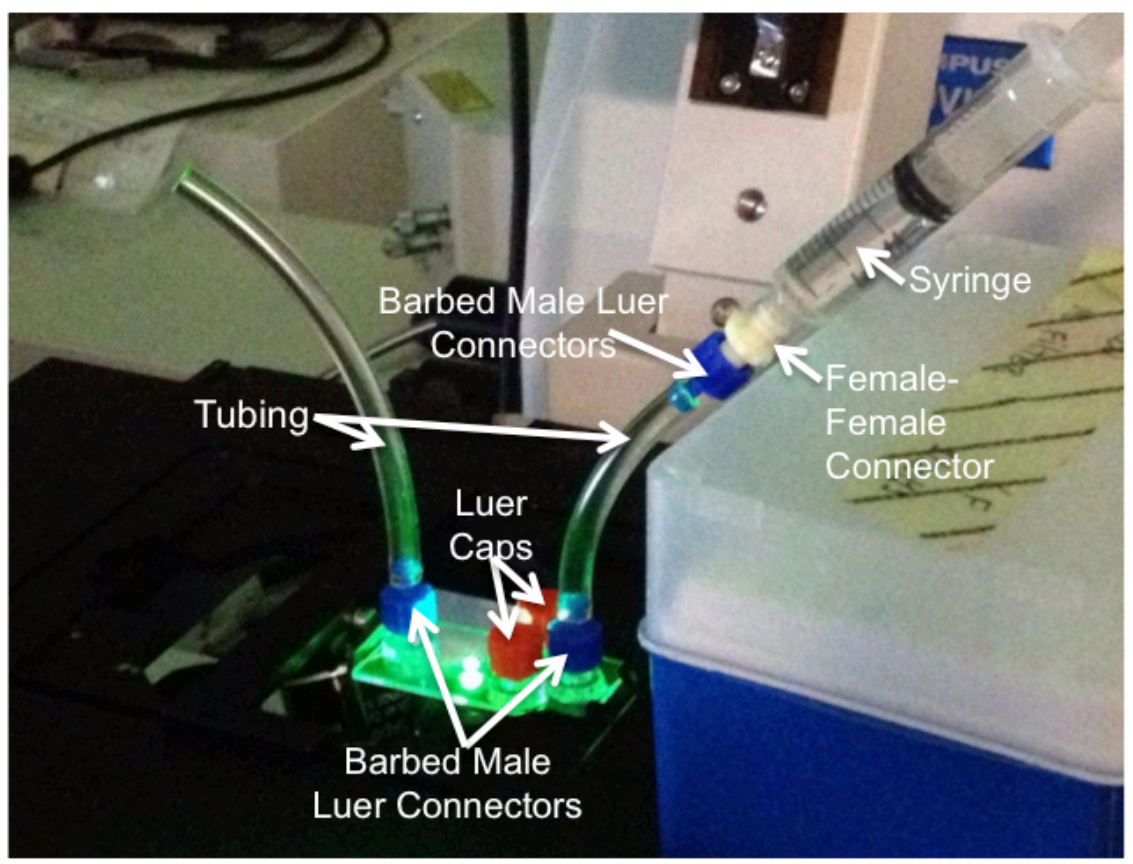

Figure 22. Pressure-driven system used to validate microfluidic chip on confocal microscope.

Deionized water was flushed through the channels to clear them using a syringe connected to the end of the tube leading from the buffer reservoir. The connection between the syringe and tubing required the use of a female to female connector and a barbed male luer connector. The sample reservoirs were capped with plugs to prevent any fluid from flowing out of the reservoirs.

Following the deionized flush, the microspheres were loaded into the microchip. A solution of $9.9 \mu \mathrm{m}$-diameter fluorescent polystyrene microspheres (Life Technologies) was created by combining $100 \mu \mathrm{L}$ of spheres with $5 \mathrm{~mL}$ of 
deionized water in a $50 \mathrm{~mL}$ conical tube then sonicated for 2 minutes in tap water to separate the particles. The solution was then drawn into a syringe and attached to the system previously described. The system was set up on a table with an Olympus Fluoview Laser Scanning Confocal Microscope (FV1000, Olympus America, Centerville, PA) and then placed in the sample holder of the microscope. The 20x objective was used for observing and imaging the microchip with microspheres. The $100 \mu \mathrm{m}$ wide by $100 \mu \mathrm{m}$ deep separation channel was found using the brightfield setting and focus was adjusted using the coarse and fine focusing knobs to clearly define the channel at an arbitrary location. Once the channel was clearly defined, the light source was changed to widefield fluorescence with a mercury arc lamp in order to get a two-dimensional image to see the spheres. A micropipette tip box was used to hold the syringe in a more vertical position in order to balance the weight and keep the microchip on the stage. Once the system was set up, the channel was highlighted for the field of vies to ease in viewing the movement of the microspheres. Next, slight pressure was applied to the syringe by hand while concurrently viewing the channel to determine when to stop applying pressure. Once the green fluorescent microspheres appeared in the field of view, pressure was released and images were taken.

Using the FluoView software (Olympus America, Centerville, PA) on the corresponding computer, the microspheres were imaged a single-plane at a time. 
The images were taken by scanning a laser across the sample on a single plane over time with a scan rate of $2 \mu \mathrm{sec} /$ frame. After the desired time, scanning ended and the image files were defaultly saved as .oib file types and then exported as .tif files to process later. The .oib file type is the standard Olympus format and is necessary to save all information related to the microscope settings. The files need to be in .tif format for processing in the desired program, ImageJ.

Next, the concentration of microspheres was increased to make it easier to view the microspheres and to see if they would alter the flow through the channels. In order to have a more microspheres in the field of view for a larger sample size, $500 \mu \mathrm{L}$ more microspheres were added to the remaining solution of approximately $3 \mathrm{~mL}$ of the prior solution in order to determine functionality of flow through the chip. The syringe was again attached to the system and images were taken. The increased concentration solution had the desired result with more microspheres appearing in the field of view. Desired images were then taken as .oib files then exported as .tif for processing using the same procedure as described previously. After testing, the channels were flushed using a syringe and deionized water. When performing both tests, the microchip would not stay on the confocal microscope stage so a new holding fixture was created.

A microchip fixture was created in order make the microchip into the same dimensions as a standard glass microscope slide to hold the microchip onto 
the confocal stage. AutoCAD was used to create two different components that would sandwich the microchip and hold it in place. The top piece (top left portion of Figure 23) fit around the chip and held the chip onto the bottom portion (top right side of Figure 23). The bottom portion contained a smaller hole that focused on the separation channel to allow for direct access for viewing on the confocal microscope. First, $1 / 4$ inch thick acrylic (McMaster Carr) was used but found to be too thick to see the channel when placed on the stage of the confocal microscope. Next, 3/32 inch thick acrylic was used and again found to be too thick when placed on the confocal microscope. Finally, 5/64 inch thick acrylic was used and was able to see the channel when positioned onto the confocal. Each time, the CAD file was loaded onto the desktop of the laptop in the IME Machine Shop (Building 41, Cal Poly) and cut using the laser cutter with the help of Mr. David Laiho (bottom of Figure 23). 


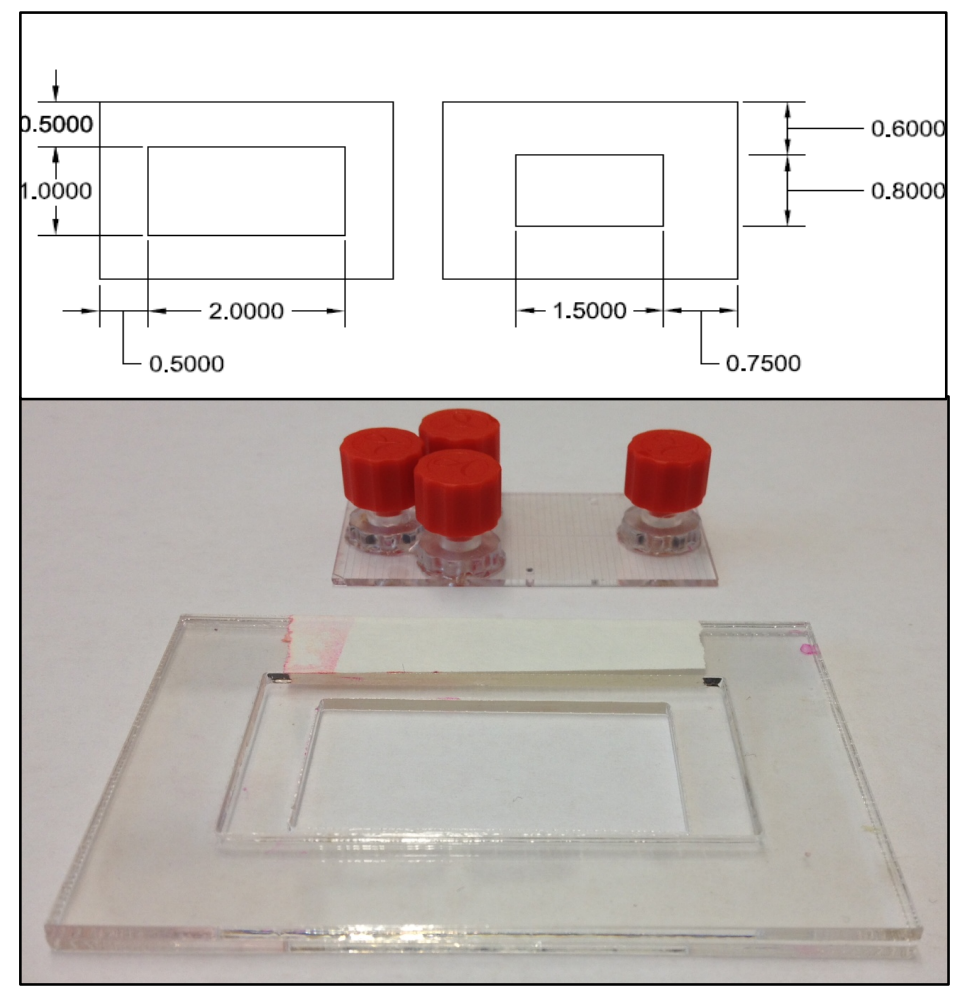

Figure 23. (Top) AutoCAD drawing of chip holder used to cut the acrylic. (Bottom) Completed chip holding fixture with lip to hold chip while fixture is held by stage of confocal microscope.

\section{ImageJ Results \& Analysis}

In order to obtain data from the images, all images were analyzed using ImageJ, a Java-based image processing program developed at the NIH used for biological image analysis [32]. The files were opened in the program and appeared as multiple images over time, called a stack. The stacks were made up of 2 channels, differential interference contrast (DIC) and fluorescent. DIC microscopy uses prisms to manipulate polarized light to increase contrast in 
samples. The channels were then separated in the stack in order to analyze the two channels independently as the spheres may look differently in the channels. To separate the channels and frames, substacks were created (Image èStacks èTools èMake Substack...) (Figure 24).

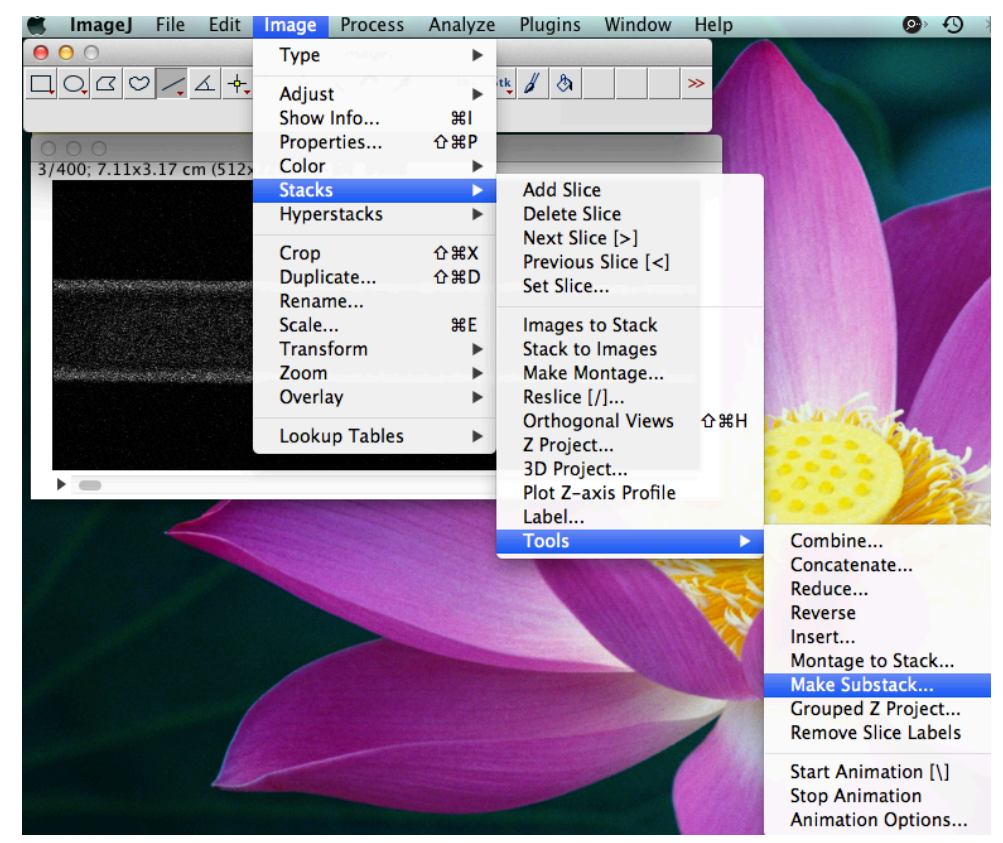

Figure 24. Creating substacks from larger stacks in order to get desired frames for another stack using the Make Substack setting.

The desired slices, corresponding with either DIC or fluorescent channel, were entered into the Substack Maker dialogue box either in a range or list format (Figure 25). A new substack of either DIC or fluorescent images was then created and further processing continued. 


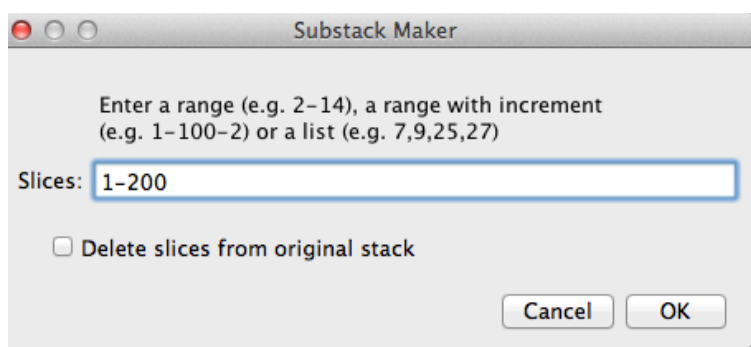

Figure 25. Substack Maker dialogue box used to designate desired slices.

Next, the scale for the images was set in order to convert measurements from pixels to micrometers. Values, taken from the computer program associated with the confocal microscope, were inputted into the set scale dialogue box (Figure 26). The program provided the conversion rate of $1.242 \mu \mathrm{m} /$ pixel that was then inserted into the corresponding places with the pixel aspect ratio of 1.0 stating that the $1.242 \mu \mathrm{m}$ corresponded to 1 pixel. The global box was then selected so all images would have the same scale used during analysis. The Set Scale dialogue box then calculated the pixel to micron ratio to be 0.805 pixels $/ \mu \mathrm{m}$. Once all the appropriate options were selected, the box was closed with $\mathrm{OK}$ and image processing continued. 


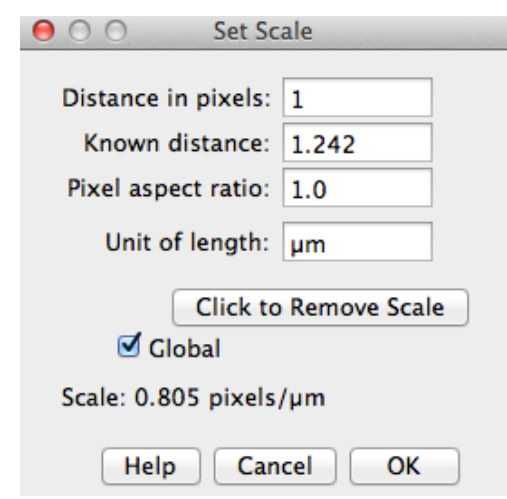

Figure 26. Setting the global scale of pixel to micron length to be applied to all images.

Next, a montage was created to show how the microspheres moved down the channel in a series of images. In ImageJ, a series, such as the substack formed in the prior section, can be created into a single image file or montage (Image èStacks èMake Montage...) (Figure 27).

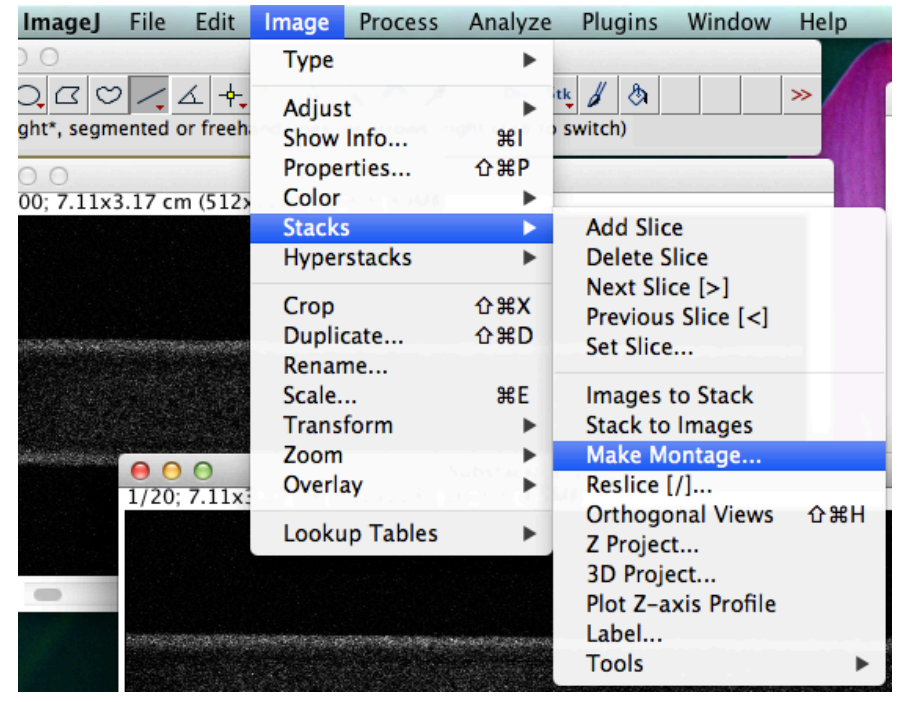

Figure 27. Creating a montage from a stack of images using the Make Montage setting. 
Once the Make Montage dialogue window opened, the desired information was filled in for each series of images (Figure 28). Since the background of all the images was black, the slices were labeled with the slice number by toggling on the Label Slices box. The Use Foreground Color box was not selected as the foreground color was black and black would not show up in the image. The column, row, first slice, last slice, and scale factor, or magnification level, numbers specified the layout of the resulting montage image. The Increment value denoted the slices or time between images displayed in the final image. The Border Width value was denoted to draw contrasting colored lines between images to easily discern the single images when combined in a single image.

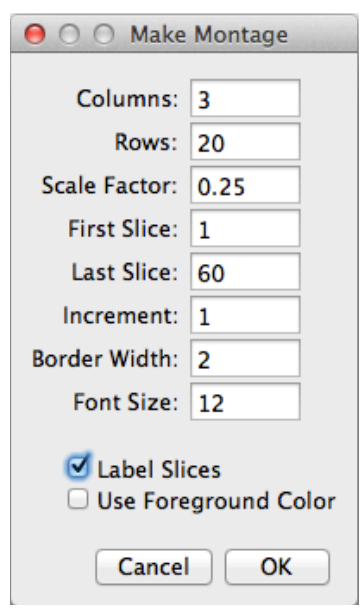

Figure 28. Settings used to create montage of images from stack. 
The montage was then created as seen below (Figure 29). This image demonstrates how the microspheres moved along the channel due to pressure applied by a hand syringe. The image shows multiple microspheres entering the field from the right side and exiting the field of view on the left side of each image. The spheres flowing in and out of the field of view demonstrates uninhibited flow through the channel after the pressure on the syringe was released. 


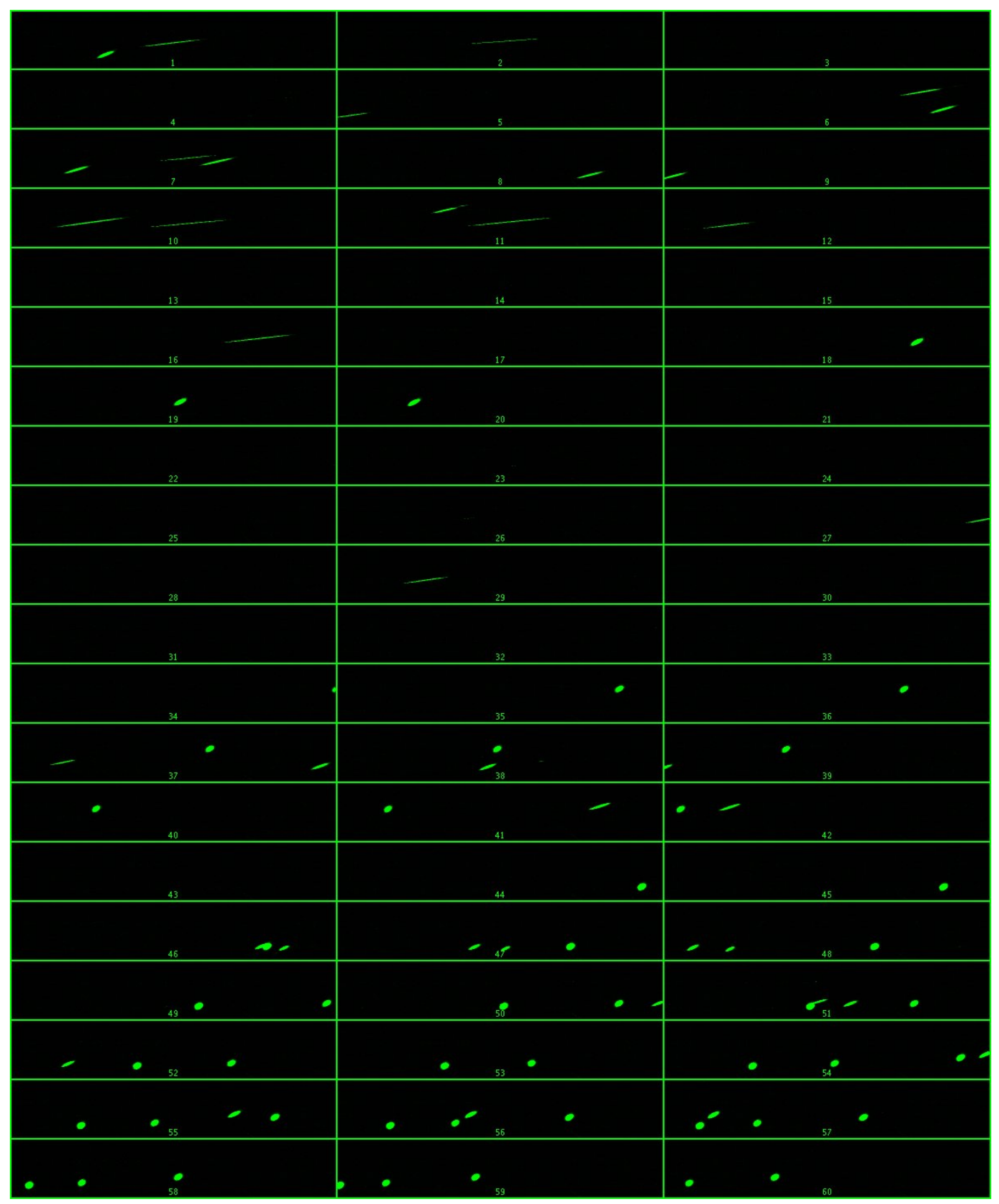

Figure 29. Montage of microspheres moving down channel of microfluidic chip due to pressure applied by a hand syringe. 
From this montage, spheres can be seen flowing into the field of view from the right and out to the left in the channel of each frame. It can be determined that the channels were clear and allowed for fluid to flow since the spheres can be seen entering and exiting the field of view.

The next step in the process was to try to determine the velocity of the spheres in the channel. In order to find the velocity of the spheres, the stack was separated into substacks if needed by following the steps previously described. The stacks or substacks were then separated into separate images in order to measure the distance traveled by the individual spheres. The individual images were then overlaid with one image over another and a line was drawn between the spheres in two locations (Figure 30). The distance was then measured by the program and recorded to use for further analysis.

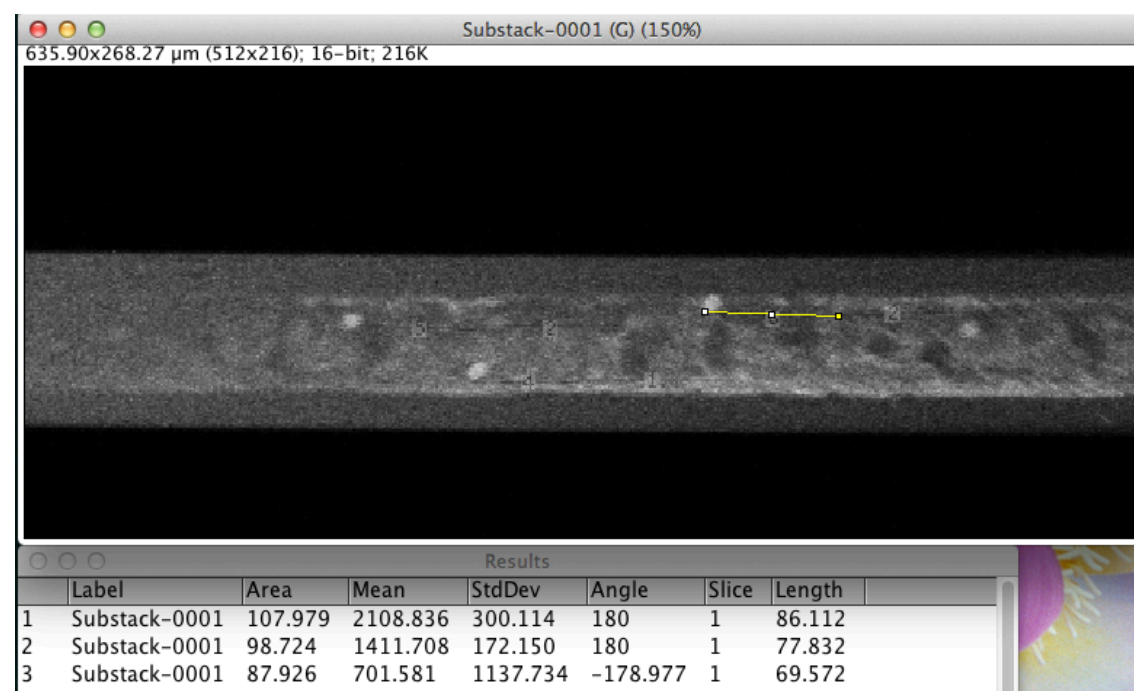

Figure 30. Measuring the distance spheres traveled between frames using ImageJ. 
A total of 70 distance values were taken from the overlaid images and then entered into Microsoft Excel to calculate the average and standard deviation of the data. The time between frames was then calculated by multiplying the scan rate of the image by the size of the image. Next, the distance traveled per frame was divided by the time between frames in order to calculate the velocity at which the spheres traveled. Finally, the average and standard deviation of the data was taken and recorded.

$$
\begin{gathered}
\text { Image size }\left(\frac{\text { pixel }}{\text { frame }}\right) \times \text { Scan Rate }\left(\frac{\mu s e c}{\text { pixel }}\right) \\
=\text { Time Between Frames }\left(\frac{\mu s e c}{\text { frame }}\right) \\
\left(512 \times 216 \frac{\text { pixel }}{\text { frame }}\right) \times\left(\frac{2 \mu \text { sec }}{\text { pixel }}\right)=221184 \frac{\mu s e c}{\text { frame }}=0.221184 \frac{\text { sec }}{\text { frame }}
\end{gathered}
$$

Table 5. Table of experimental values for pressure-driven flow of $10 \mathrm{um}$ microspheres.

\begin{tabular}{|c|c|c|}
\hline & Average & Standard Deviation \\
\hline Distance $(\mu \mathrm{m})$ & 87.86 & 68.62 \\
\hline Velocity $(\mu \mathrm{m} / \mathrm{sec})$ & 397.22 & 310.22 \\
\hline
\end{tabular}

Calculating the velocity of the spheres traveling down the channel provided experience with ImageJ to develop a method to determine the velocity at 
which particles travel through channels in a microchip. With this knowledge, further progress was possible as the microchip was validated.

MDA

The next test was to perform the pressure-driven microsphere test again, but replaced the microspheres with a solution of MDA and TBA in deionized water used to verify that the MDA solution would flow through the channels and pressure driven flow could be used if voltage driven flow failed. Malondialdehyde tetrabutylammonium salt and 2-Thiobarbituric acid (Sigma-Aldrich, St. Louis, MO) were handled inside the fume hood using appropriate protective precautions including gloves, long sleeved shirts, long pants, closed toe shoes, and goggles. Three concentrations of MDA solutions were created, $10 \mathrm{mM}, 20 \mu \mathrm{M}$, and $0.02 \mu \mathrm{M}$, following the Fluorometric TBARS Miccroplate Assay Kit protocol (Oxford Biomedical Research Inc., 2012). The solutions were used to run through the system in order to determine if any concentration would block the flow through the channel and that different concentrations could be detected. The solutions were also used in later experiments involving MDA solutions. A 10mM MDA solution was first made by taring the scale with a $10 \mathrm{~mL}$ conical tube then weighing out approximately 0.0313 grams of the MDA salt into the conical tube. Next, $10 \mathrm{~mL}$ of deionized water was added to the conical tube and vortexed for 
approximately 30 seconds. Then, the $10 \mathrm{mM}$ solution was diluted 1:500 in deionized water by adding $20 \mu \mathrm{L}$ of $10 \mathrm{mM}$ MDA to $9.98 \mathrm{~mL}$ of deionized water to make a $20 \mu \mathrm{M}$ MDA solution. The solution was further diluted 1:1,000 in deionized water by adding $10 \mu \mathrm{L}$ of $20 \mu \mathrm{M}$ MDA solution to $9.99 \mathrm{~mL}$ of deionized water to make a $0.02 \mu \mathrm{M}$ MDA solution.

The TBA assay was then prepared by tarring the scale with a $10 \mathrm{~mL}$ conical tube then weighing out approximately 1.0 gram of the TBA powder into the conical tube. Next, $10 \mathrm{~mL}$ of deionized water was added to the conical tube and shaken by hand until the powder had dissolved. Both the MDA and TBA solutions were made directly prior to use.

The microfluidic chip was again set up on the confocal microscope using the fabricated holder and taped together (Figure 31). Male luer connectors were attached to the microchip at the buffer and buffer waste reservoirs. A male to female luer connector was then attached to the chip and a female to female elbow connector for both ends of the chip. Next, a male to barbed luer connector was attached to a short piece of $1 / 8$ inch tubing. The waste end then had a paper towel under the tube to collect the waste fluid. The buffer input end had had another male barbed luer connector to a female to female connection for the syringe attachment. The sample and sample waste reservoirs had caps to prevent fluid from flowing into the channels. 


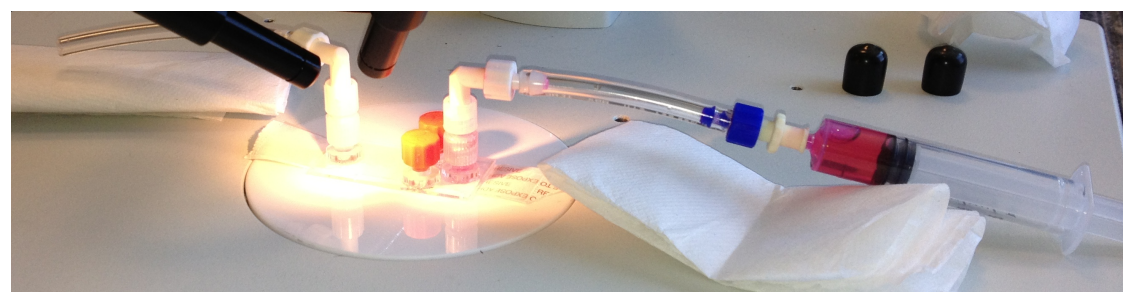

Figure 31. Set up of pressure-driven MDA test without chip holder and not on confocal microscope.

The tests followed the Fluorometric TBARS Miccroplate Assay Kit protocol (Oxford Biomedical Research Inc., 2012) to test different concentrations of MDA in solution. The first test was composed of $1 \mathrm{~mL}$ of $10 \mathrm{mM}$ MDA solution with $0.5 \mathrm{~mL}$ of TBA assay in a $50 \mathrm{~mL}$ conical tube which was then vortexed ( $\sim 30$ seconds). Once vortexed, the solution turned from clear to fuchsia. First, the channels of the microchip were primed with deionized water from a syringe. Next, the syringe was filled with the TBA/MDA solution and slowly injected into the system while on the confocal microscope stage. Minor pressure by handheld injection was used to inject the sample into the microchip. Desired images were taken at $20 \mathrm{x}$ with a scan rate of $2 \mu \mathrm{sec} /$ frame and saved as .oib files and exported as .tif files. The files must be saved as .oib as it is the standard Olympus format required to save the settings for the microscope. The channels were then cleared with deionized water.

The chip was then tested with a lower concentration of MDA solution in order to determine if the chip could be used to detect lower MDA concentrations 
in a sample. Another MDA/TBA solution was made with the $1.0 \mathrm{~mL}$ of the 0.02 $\mu \mathrm{M}$ MDA solution and $0.5 \mathrm{~mL}$ of the TBA assay in a $50 \mathrm{~mL}$ conical tube which was then vortexed ( $\sim 30$ seconds) following the protocol. Once vortexed and approximately 3 minutes, the solution turned from clear to fuchsia. The syringe was then filled with the TBA/MDA solution and connected to the microchip. Again, the solution was injected into the microchip using handheld pressure and desired images were taken at 20x. The channels were again cleaned with deionized water from a syringe connected to the system.

Finally, the chip was again tested with the lowest concentration of MDA solution in order to determine if the chip could be used to detect a significantly lower MDA concentration in a sample. A solution of $2.0 \mathrm{~mL} 10 \mathrm{mM}$ MDA solution and $0.5 \mathrm{~mL}$ of TBA assay were combined in a $50 \mathrm{~mL}$ conical tube which was then vortexed ( $\sim 30$ seconds). Once vortexed, the solution turned from clear to fuchsia after approximately 2 minutes. Again, a syringe was filled with the TBA/MDA solution and connected to the microchip. The solution was then injected into the microchip using handheld pressure and desired images were taken at 20x. The channels were again cleaned with deionized water from a syringe connected to the system. 
ImageJ Results \& Analysis

Image J was used again to analyze the images taken of the pressure-driven MDA flow in order to verify the presence of MDA flowing through the channel. With the existence of MDA in the channel, the chip can continue to be tested with electrophoresis and if voltage-driven flow fails, pressure-driven flow can be used. Before any MDA was injected into the channel, an image of the channel was taken (Figure 32). The sides of the channel were defined by the thicker, more intense green light and the light inside the channel may be caused by autofluorescence of the fused silica chip.

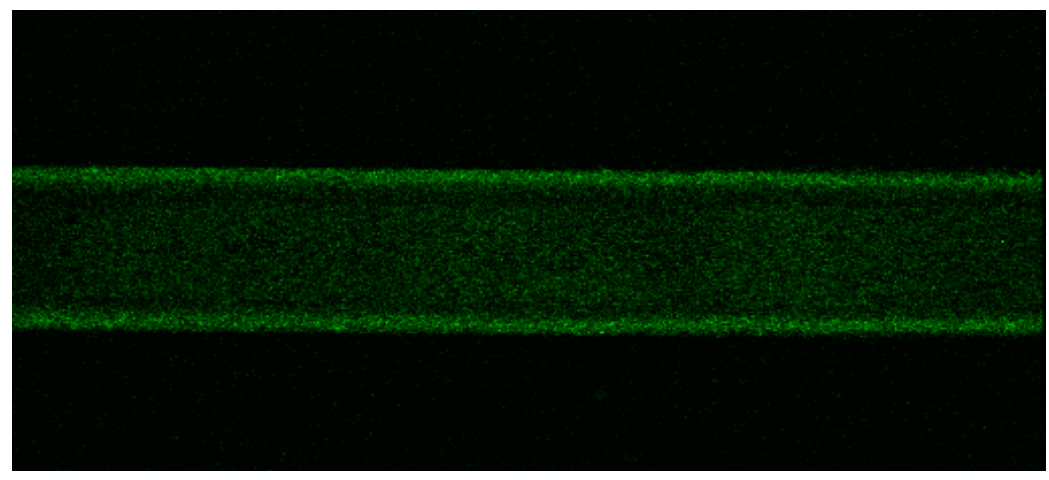

Figure 32. Image of empty channel after washing with deionized water.

Next, the scale for the images was set in order to convert measurements from pixels to micrometers. Values, taken from the computer program associated with the confocal microscope, were inputted into the set scale dialogue box (Figure 33). The program provided the conversion rate of $1.242 \mu \mathrm{m} / \mathrm{pixel}$ that was then inserted into the corresponding places with the pixel aspect ratio of 1.0 
stating that the $1.242 \mu \mathrm{m}$ corresponded to 1 pixel. The global box was then selected so all images would have the same scale used during analysis. The Set Scale dialogue box then calculated the pixel to micron ratio to be 0.805 pixels $/ \mu \mathrm{m}$. Once all the appropriate options were selected, the box was closed with OK and image processing continued.

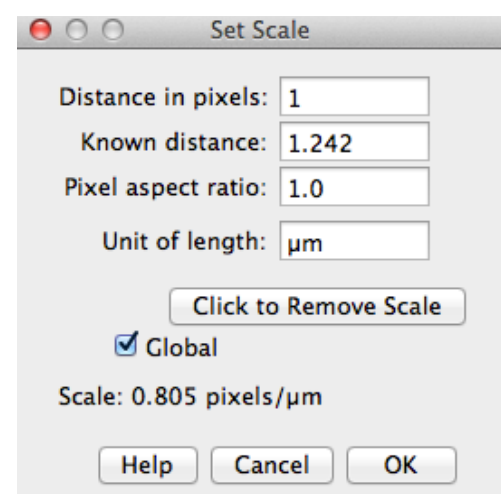

Figure 33. Setting the global scale of pixel to micron length to be applied to all images.

The channels were then separated in the stack in order to analyze the two channels independently as the MDA may look differently in the channels. To separate the channels and frames, substacks were created (Image èStacks èTools èMake Substack...) (Figure 34). 


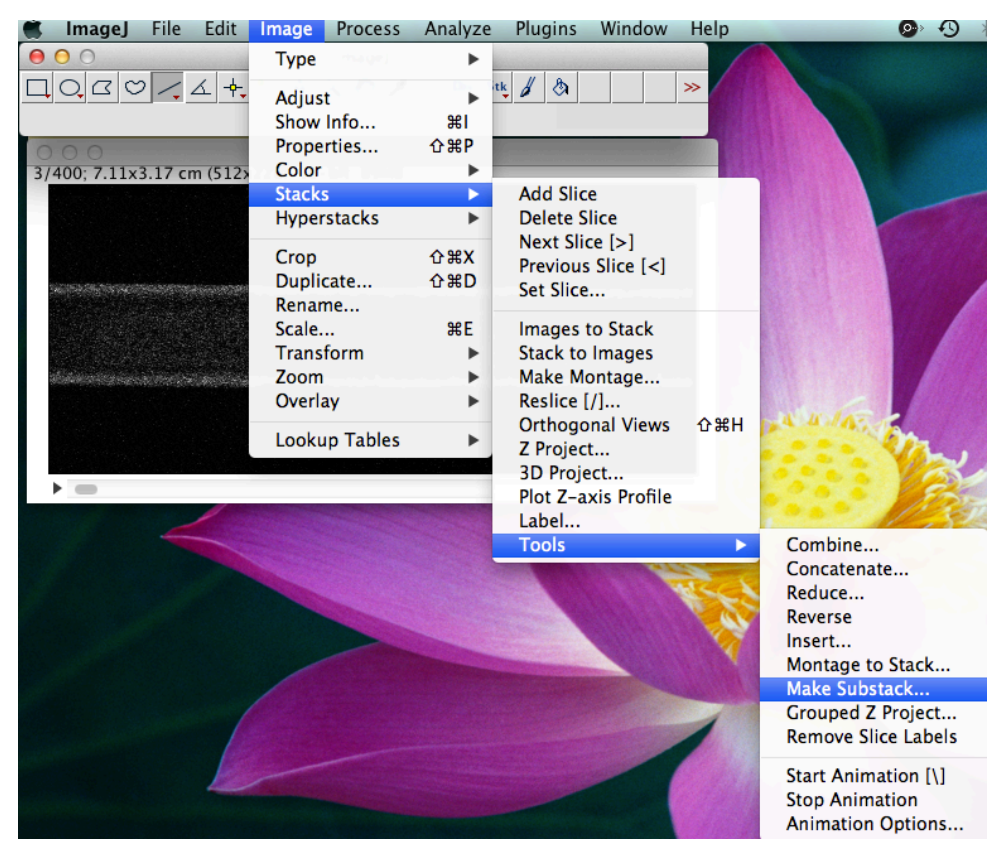

Figure 34. Creating substacks from larger stacks in order to get desired frames for another stack using the Make Substack setting.

The desired slices, corresponding with either DIC or fluorescent channel, were entered into the Substack Maker dialogue box either in a range or list format (Figure 35). A new substack of either DIC or fluorescent images was then created and further processing continued in order to observe the MDA flow down the channel.

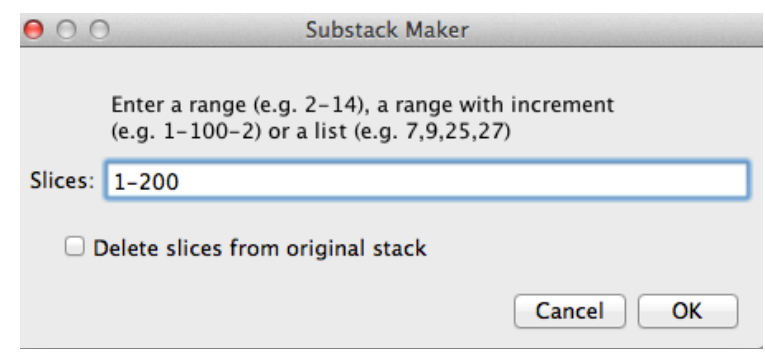

Figure 35. Substack Maker dialogue box used to designate desired slices. 
Next, a montage was created to show how the MDA/TBA solution moved down the channel in a series of images. In ImageJ, a series, such as the substack formed in the prior section, can be created into a single image file or montage (Image èStacks èMake Montage...) (Figure 36).

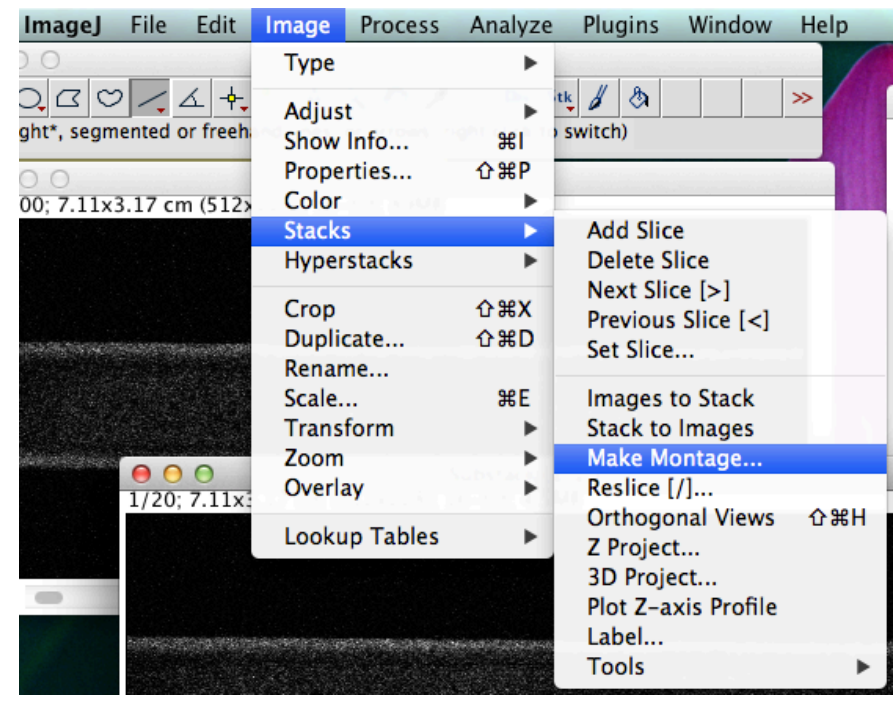

Figure 36. Creating a montage from a stack of images using the Make Montage setting.

Once the Make Montage dialogue window opened, the desired information was filled in for each series of images (Figure 37). Since the background of all the images was black, the slices were labeled with the slice number by toggling on the Label Slices box. The Use Foreground Color box was not selected as the foreground color was black and black would not show up in the image. The column, row, first slice, last slice, and scale factor, or magnification level, numbers specified the layout of the resulting montage image. The Increment value 
denoted the slices or time between images displayed in the final image. The Border Width value was denoted to draw contrasting colored lines between images to easily discern the single images when combined in a single image.

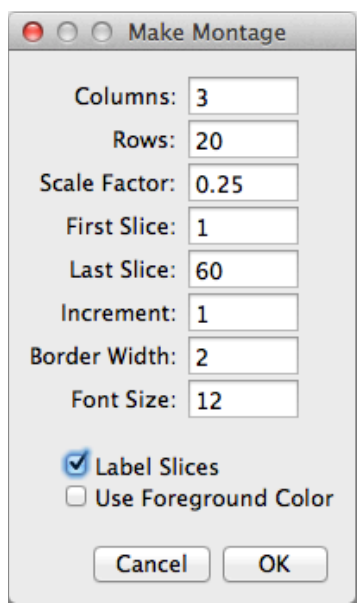

Figure 37. Settings used to create montage of images from stack.

The montage was then created in order to see when the MDA entered and exited the field of view (Figure 38). This image demonstrates how the MDA, appears green in the images, moved along the channel due to pressure applied by a hand syringe. The image shows the MDA entering the field from the right side, in images 120 and 132, and exiting the field of view on the left side, in image 121, and continuously flowing, images 132 through 140 . The MDA flowing in and out of the field of view, as seen by the green areas appearing and disappearing, demonstrates uninhibited flow through the channel after the pressure on the 
syringe was released. The next step in the process was to try to determine the velocity of the MDA in the channel.

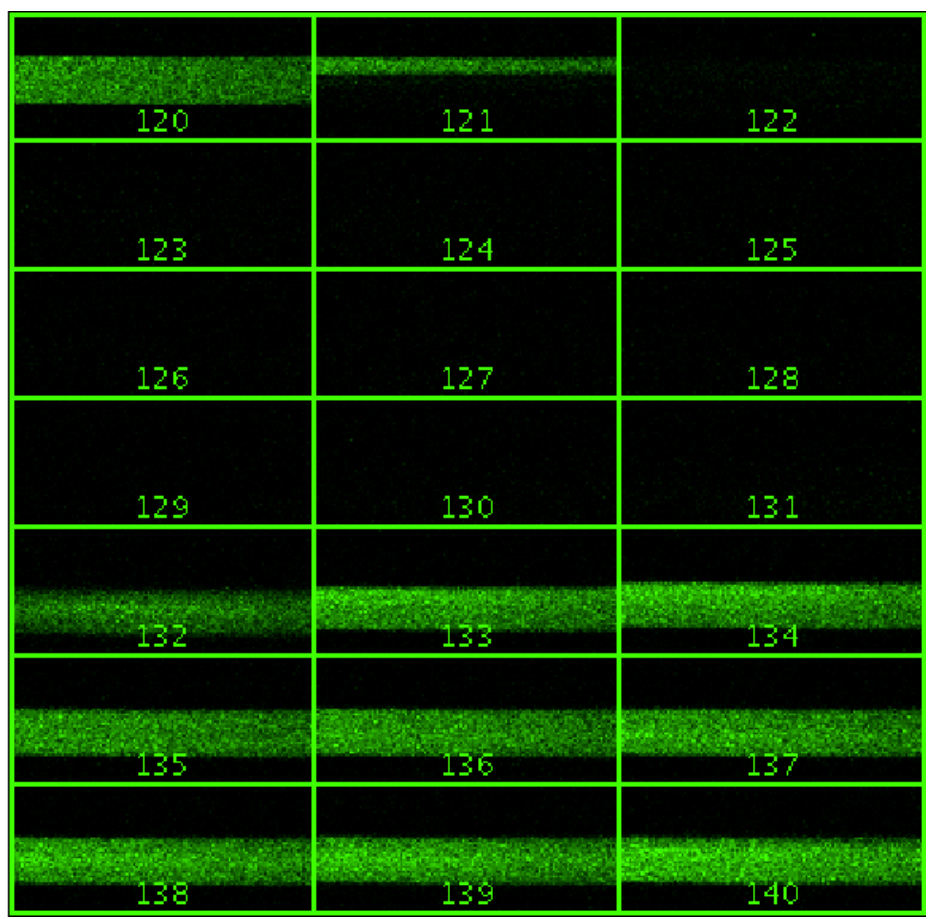

Figure 38. Montage of slices 120-140 of 200 image stack, showing MDA fluid in the channel, leaving the channel, and re-entering the channel.

In order to find the velocity of the MDA, the stack was separated into substacks if needed by following the steps previously described. The stacks or substacks were then separated into separate images in order to measure the distance traveled by the MDA. The individual images were then overlaid with one image over another and a line was attempted to be drawn between the areas of MDA in two locations. Unfortunately, in all the images, it was impossible to 
distinguish any locations on the images in which to measure the distance the MDA traveled between frames (Figure 39). Without knowing the distance traveled between frames, velocity could not be accurately calculated. The MDA may have been moving at a faster velocity than that of the $2 \mu \mathrm{s} /$ pixel scan rate, therefore it was unable to pick a point in two images that could be used to measure the distance traveled.

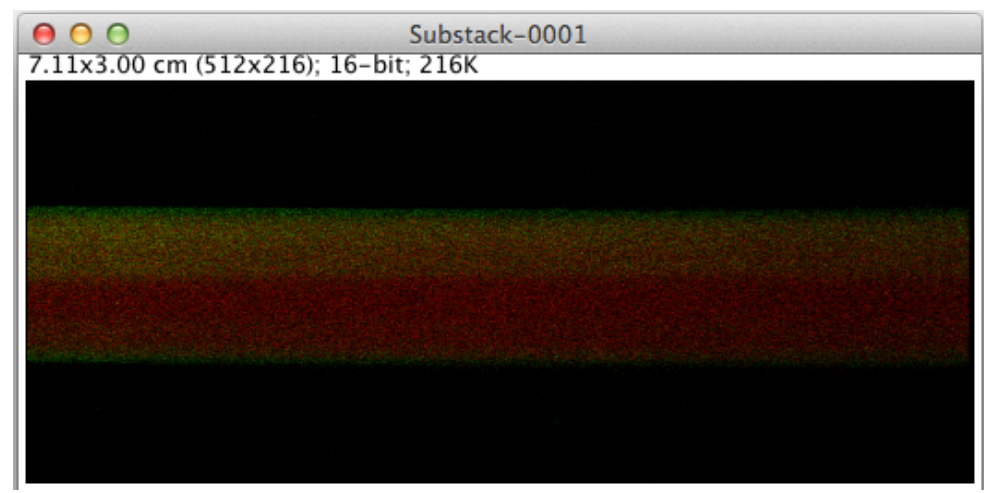

Figure 39. Attempting to measure the distance spheres traveled between frames using ImageJ.

Although the exact velocity could not be calculated, the time between frames was calculated by multiplying the scan rate of the image by the size of the image. Next, the distance traveled per frame was assumed to be greater than or equal to the width of the field of view. This distance was then divided by the time between frames in order to calculate the approximate velocity at which the 
spheres traveled (Table 6). This value was not accurate but was calculated as practice for later measurements.

$$
\begin{gathered}
\text { Image size }\left(\frac{\text { pixel }}{\text { frame }}\right) \times \text { Scan Rate }\left(\frac{\mu \text { sec }}{\text { pixel }}\right) \\
=\text { Time Between Frames }\left(\frac{\mu s e c}{\text { frame }}\right) \\
\left(512 \times 228 \frac{\text { pixel }}{\text { frame }}\right) \times\left(\frac{2 \mu \mathrm{sec}}{\text { pixel }}\right)=233472 \frac{\mu \mathrm{sec}}{\text { frame }}=0.233472 \frac{\mathrm{sec}}{\text { frame }}
\end{gathered}
$$

Table 6. Table of approximated values for pressure-driven flow of MDA solution.

\begin{tabular}{|c|c|}
\hline Distance $(\mu \mathrm{m})$ & Velocity $(\mu \mathrm{m} / \mathrm{sec})$ \\
\hline 512 & 2192.98 \\
\hline
\end{tabular}

Although calculating the velocity of the MDA traveling down the channel was not possible, the test did demonstrate the ability to view MDA in the channel. It also provided experience with ImageJ to develop a method to determine the velocity at which MDA travel through channels in a microchip. Furthermore, the images demonstrate the ability to detect MDA using a confocal microscope. With this knowledge, further progress was possible as the microchip was validated. It was also determined that pressure-driven flow of MDA could be used for detection in the future if voltage-driven flow failed. 


\section{Model of Microchip Capillary Electrophoresis System}

The microchip was then set up for capillary electrophoresis as described in the Final Design Proof of Concept (Figure 21 and 40). Electrodes were created by stripping wires to expose the metal from a pre-made kit for gel electrophoresis (Bio-Rad, Hercules, CA). The stripped wires were then threaded through the barbed end of male luer connectors and attached via heat shrink wrap. Alligator clips at the other end were able to connect to banana plugs leading to the voltage source. A female to male connector attached to the chip and a female to female connector. Next, an all female t-connector was attached with the electrode male luer connector and syringe were attached to the top ports for the buffer input well and just an electrode connector at the waste end. The cathode electrode was placed at the waste well and the anode was placed at the buffer/sample input well.

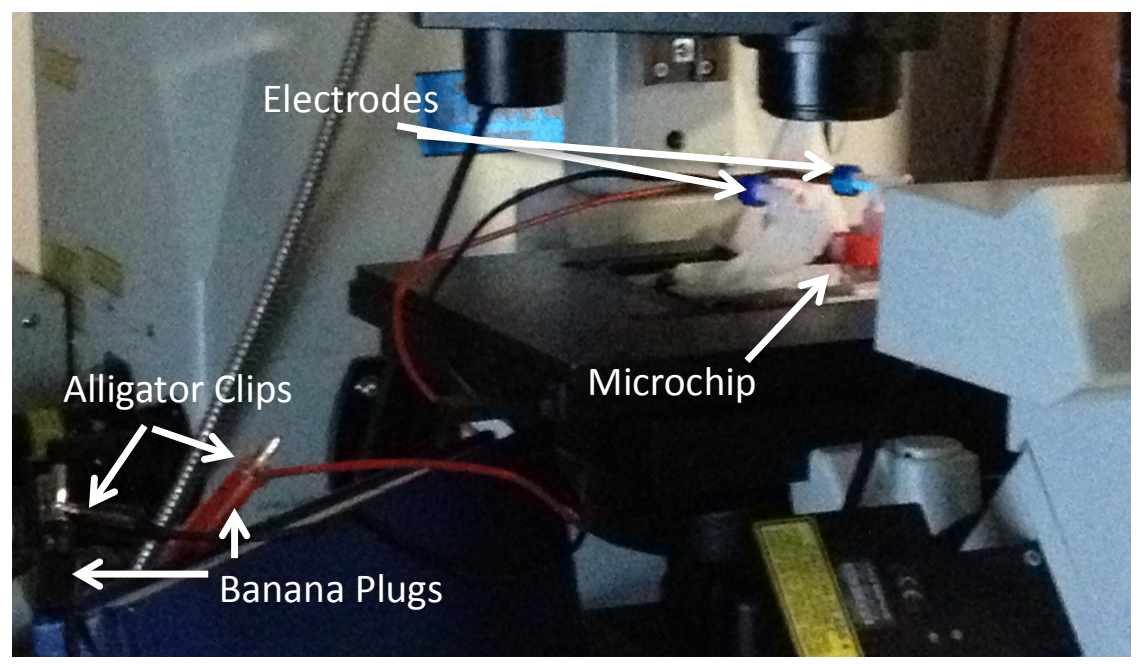

Figure 40. Labeled image of the CE system setup with the microchip that was used in the previous experiments. 


\section{Confocal Microscope Imaging}

After the CE system was set up with the microchip, the system was placed on the microscope in order to view the microspheres or MDA solution flow through the channel.

\section{Microspheres}

The purpose of this experiment was to learn if the CE system was set up correctly and to learn techniques to make the process smoother. The microchip was placed onto the confocal microscope with the syringe containing $600 \mu \mathrm{L}$ microspheres $/ 5 \mathrm{~mL}$ deionized water prepared with the same preparation as previously described. An arbitrary location along the channel was again found using the same process as previously described. An image was then taken as a control for the channel. Pressure was slowly applied to the syringe by hand to inject the channel with the microspheres. The voltage source was then turned on and voltage was applied starting with $20 \mathrm{~V}$, then $40 \mathrm{~V}$, and finally $48.8 \mathrm{~V}$. The first two voltages, $20 \mathrm{~V}$ and $40 \mathrm{~V}$, were arbitrarily selected and the $48.8 \mathrm{~V}$ was selected as it was the highest voltage possible with the voltage source provided. 


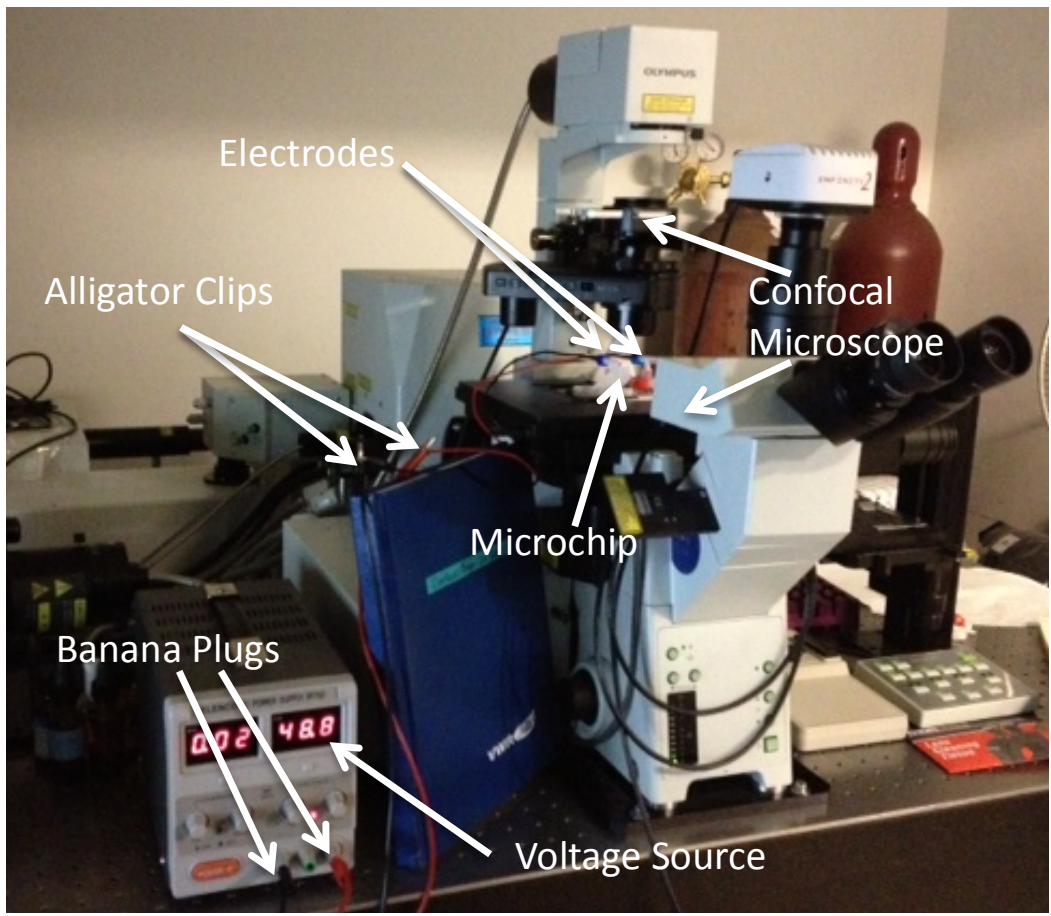

Figure 41. Bench model of CE system setup on the confocal microscope with the voltage source.

Images were taken using the FluoView software on the corresponding computer to view the microspheres moving along the channel a single-plane at a time. The images were taken by scanning a laser across the sample on a single plane over time with a scan rate of $2 \mu \mathrm{s} /$ pixel. After the desired time, scanning ended and the image files were defaultly saved as .oib file types and then exported as .tif files to process later. The .oib file type is the standard Olympus format necessary to save all information related to the microscope settings. The files need to be in .tif format for processing in the desired program, ImageJ. 
When the syringe remained in the system, the microspheres appeared to flow in the direction reverse to the polarity of the charge. The syringe was then removed and the spheres then flowed in the direction of the polarity of the charge and some even stopped. When the syringe was removed, the residual pressure from the pressure was able to quickly dissipate causing the spheres to flow in the direction of the applied voltage. With the syringe removed from the system, a test was performed to see if the charge was directing the flow of the microspheres.

The purpose of the secondary test was to determine if the directionality of the microspheres was based on the charge applied to the system. In order to decide if the spheres flow was based on the applied charge, the directionality of the charge was reversed to verify that the spheres would reverse flow. The system was turned on with the original charge directionality of cathode, positive charge, in the waste well and anode, negative charge, in the sample well of the chip. After a few minutes, the voltage source was then turned off and the electrodes were switched at the alligator-banana clip end and the voltage turned back on with the charge in the reverse directions. The microspheres changed direction to flow toward the cathode which was located in the sample well. This voltage shift was attempted multiple times with at various times between shifting voltages, with the same result. Voltage was then turned off and the microspheres continued to flow down the channel. The channel was then cleaned with deionized water via a syringe injected with hand pressure. 
ImageJ Results \& Analysis

ImageJ was used again to analyze the images taken of the voltage-driven microspheres in order to determine if the CE system was properly set up to drive the microspheres through the channel.

First, the image files were opened in ImageJ the scale for the images was set in order to convert measurements from pixels to micrometers. Values, taken from the computer program associated with the confocal microscope, were inputted into the set scale dialogue box (Figure 42). The program provided the conversion rate of $1.242 \mu \mathrm{m} / \mathrm{pixel}$ that was then inserted into the corresponding places with the pixel aspect ratio of 1.0 stating that the $1.242 \mu \mathrm{m}$ corresponded to 1 pixel. The global box was then selected so all images would have the same scale used during analysis. The Set Scale dialogue box then calculated the pixel to micron ratio to be 0.805 pixels $/ \mu \mathrm{m}$. Once all the appropriate options were selected, the box was closed with OK and image processing continued. 


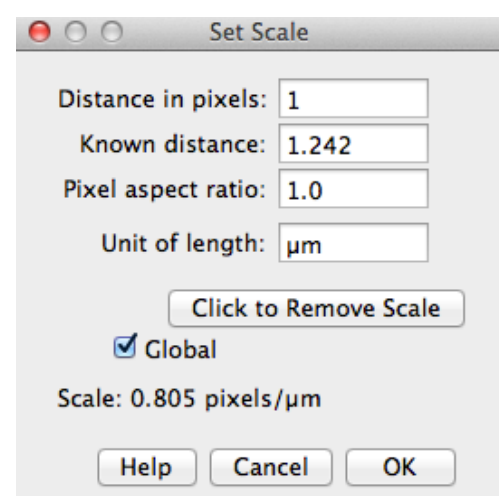

Figure 42. Setting the global scale of pixel to micron length to be applied to all images.

The channels were then separated in the stack in order to analyze the two channels independently as the microspheres may look differently in the channels. To separate the channels and frames, substacks were created (Image èStacks èTools èMake Substack...) (Figure 43). 


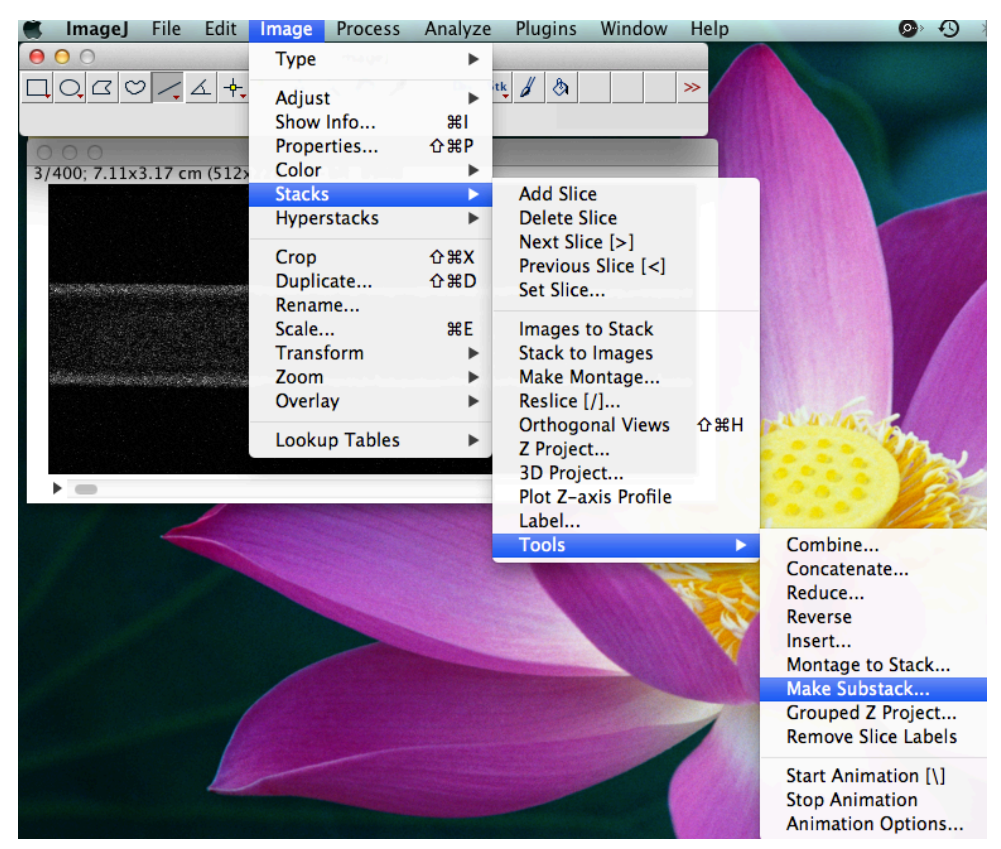

Figure 43. Creating substacks from larger stacks in order to get desired frames for another stack using the Make Substack setting.

The desired slices, corresponding with either DIC or fluorescent channel, were entered into the Substack Maker dialogue box either in a range or list format (Figure 44). A new substack of either DIC or fluorescent images was then created and further processing continued in order to observe the microspheres flow down the channel.

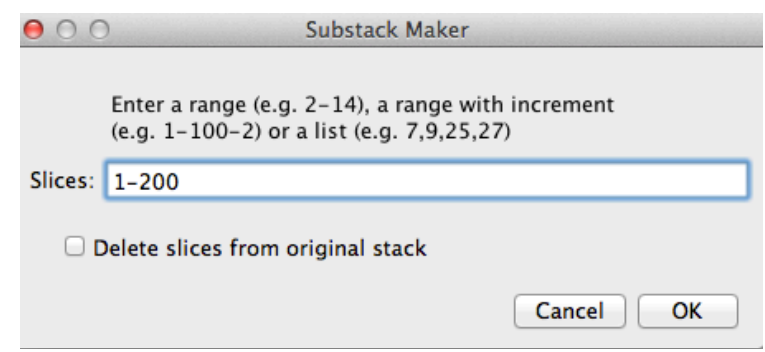

Figure 44. Substack Maker dialogue box used to designate desired slices. 
Next, a montage was created to show how the microspheres moved down the channel in a series of images. In ImageJ, a series, such as the substack formed in the prior section, can be created into a single image file or montage (Image èStacks èMake Montage...) (Figure 45).

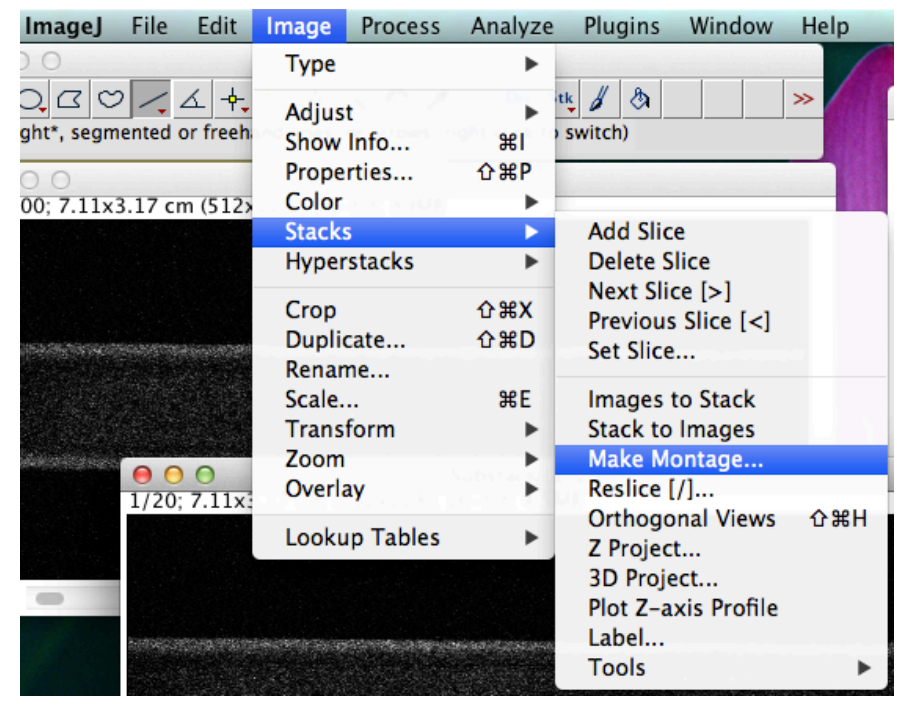

Figure 45. Creating a montage from a stack of images using the Make Montage setting.

Once the Make Montage dialogue window opened, the desired information was filled in for each series of images (Figure 46). Since the background of all the images was black, the slices were labeled with the slice number by toggling on the Label Slices box. The Use Foreground Color box was not selected as the foreground color was black and black would not show up in the image. The column, row, first slice, last slice, and scale factor, or magnification level, numbers specified the layout of the resulting montage image. The Increment value 
denoted the slices or time between images displayed in the final image. The Border Width value was denoted to draw contrasting colored lines between images to easily discern the single images when combined in a single image.

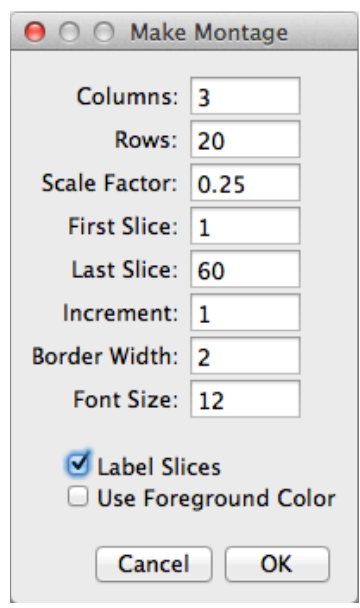

Figure 46. Settings used to create montage of images from stack.

The montage was then created in order to see when the microspheres entered and exited the field of view and the directionality of the microspheres (Figure 47). 


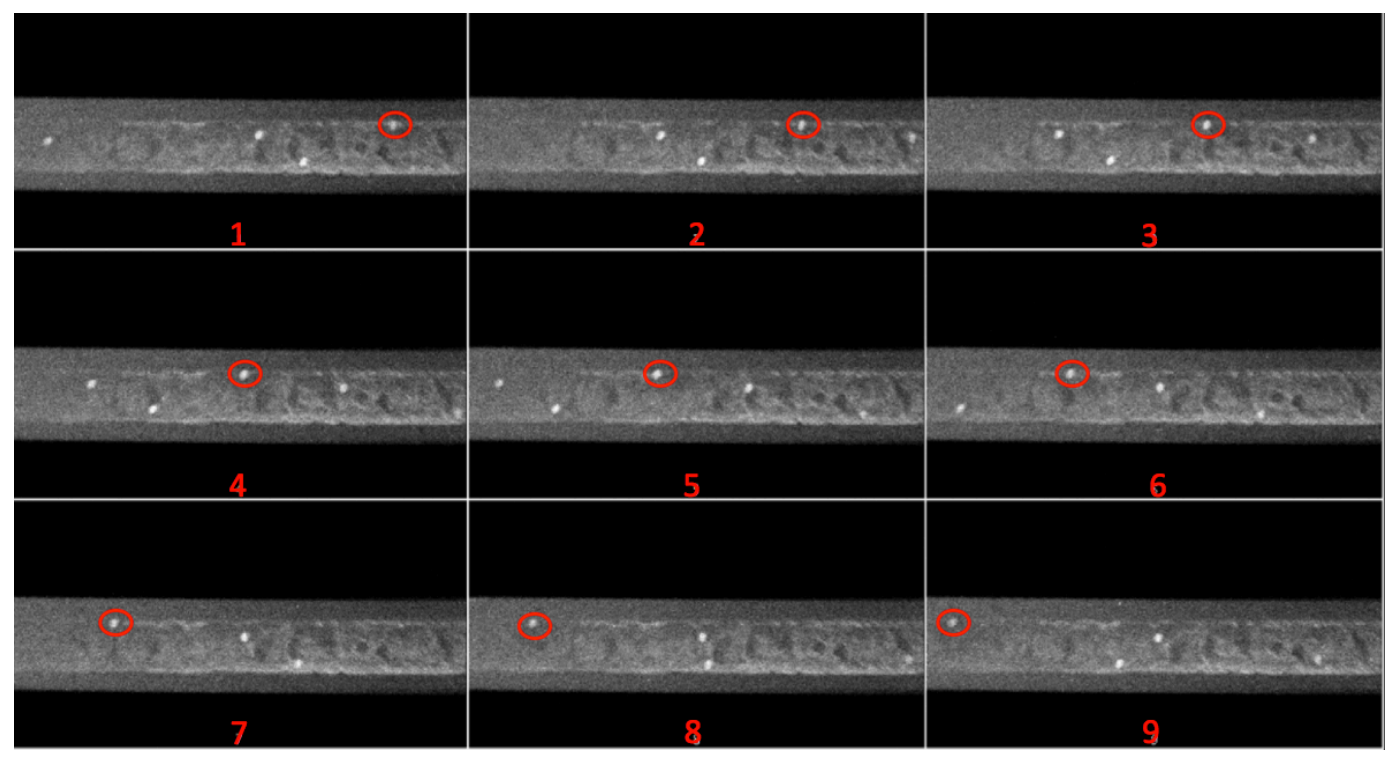

Figure 47. Montage of images, in numerical order, demonstrating the flow of a single microsphere, circled in red, flowing down the channel, from right to left of the channel, in the same direction as the electric field.

This image demonstrates how the microspheres moved along the channel in the same direction as the electric field, due either to pressure applied by the hand syringe or by the applied electric field. The sequence of images follows in numerical order starting in the first image in the top left corner and ending in the bottom right corner. Each image shows the microspheres entering the field from the right side of the channel in each image, and exiting the field of view on the left side of each image, (Figure 47). The next step in the process was to try to determine the velocity of the spheres in the channel.

In order to find the velocity of the microspheres, the stack was separated into substacks if needed by following the steps previously described. The stacks or substacks were then separated into separate images in order to measure the 
distance traveled by the individual spheres. The individual images were then overlaid with one image over another and a line was attempted to be drawn between the areas of microspheres in two locations (Figure 48). The distance was then measured by the program and recorded to use for further analysis.

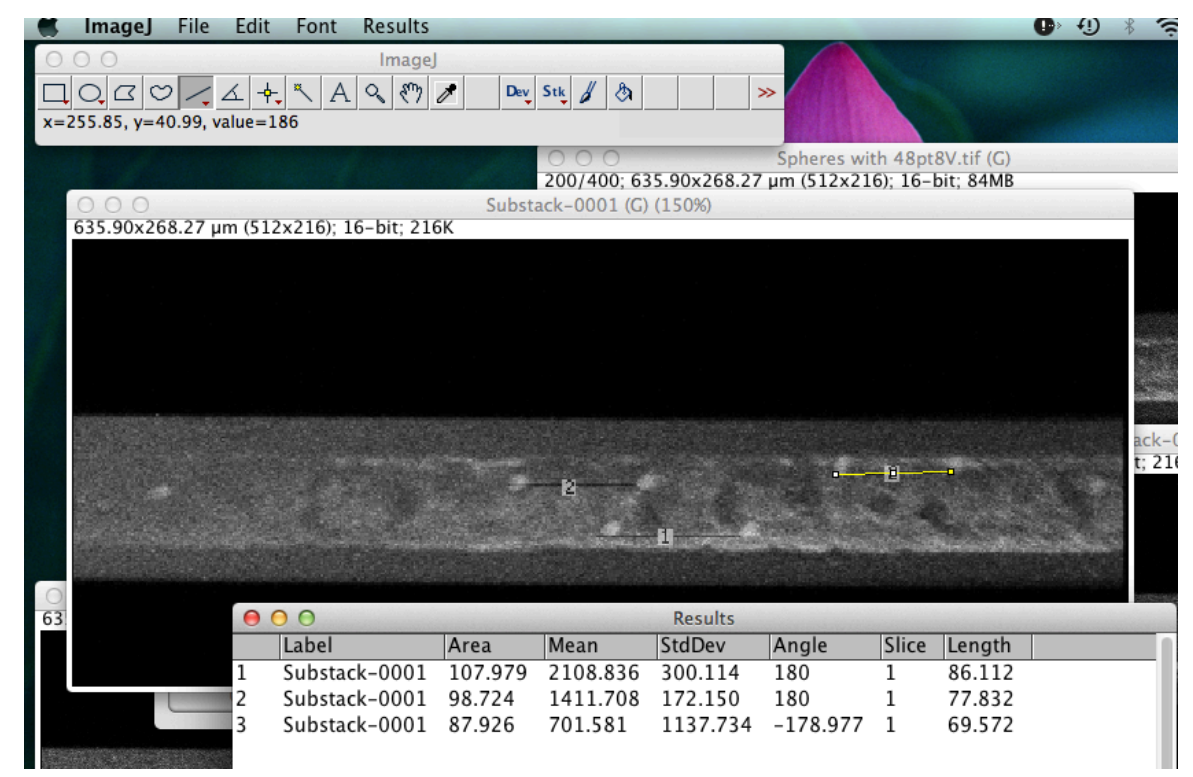

Figure 48. Measuring the distance between microspheres flowing down the channel due to voltage-flow.

A total of 120 values were taken from the overlaid images and then entered into Microsoft Excel to calculate the average and standard deviation of the data. The time between frames was then calculated by multiplying the scan rate of the image by the size of the image. Next, the distance traveled per frame was divided by the time between frames in order to calculate the velocity at which the 
spheres traveled. Finally, the average and standard deviation of the data was taken and recorded and compared with the calculated values (Table 7).

$$
\begin{gathered}
\text { Image size }\left(\frac{\text { pixel }}{\text { frame }}\right) \times \text { Scan Rate }\left(\frac{\mu s e c}{\text { pixel }}\right) \\
=\text { Time Between Frames }\left(\frac{\mu s e c}{\text { frame }}\right) \\
\left(512 \times 216 \frac{\text { pixel }}{\text { frame }}\right) \times\left(\frac{2 \mu \mathrm{sec}}{\text { pixel }}\right)=221184 \frac{\mu \mathrm{sec}}{\text { frame }}=0.221184 \frac{\mathrm{sec}}{\text { frame }}
\end{gathered}
$$

Table 7. Table of values for voltage-driven flow of $10 \mathrm{um}$ microspheres.

\begin{tabular}{|c|c|c|}
\hline & Average & Standard Deviation \\
\hline Distance $(\mu \mathrm{m})$ & 72.12 & 11.88 \\
\hline $\begin{array}{c}\text { Velocity } \\
(\mu \mathrm{m} / \mathrm{sec})\end{array}$ & 326.05 & 53.70 \\
\hline $\begin{array}{c}\text { Theoretical } \\
\text { Velocity } \\
(\mu \mathrm{m} / \mathrm{sec})\end{array}$ & $8.159 \times 10^{-3}$ & \\
\hline Percent Error & $39961 \%$ & \\
\cline { 1 - 2 } & &
\end{tabular}

Calculating the velocity of the spheres traveling down the channel allowed for a comparison between the calculated velocity versus the experimental values in order to better understand the experimental CE system. The calculated velocity was calculated using the Debye-Huckel-Henry theory to calculate the electrophoretic mobility, $\mu_{\mathrm{ep}}$, which was used to calculate the electrophoretic velocity, $\mathrm{v}_{\mathrm{ep}}[36]$ (Table 8). 
Table 8. Variables used to calculate velocity of voltage-driven microspheres.

\begin{tabular}{|l|l|l|}
\hline Variable & Definition & Assumed Value \\
\hline $\mathrm{q}$ & Net charge of particle & $6 \times 10^{-19} \mathrm{C}$ \\
\hline $\mathrm{R}$ & Stokes radius of particle & $5 \mu \mathrm{m}$ \\
\hline$\eta$ & Viscosity of solution & $1.002 \mathrm{mPa}-\mathrm{s}$ \\
\hline $\mathrm{E}$ & Electric Field Strength & $1284.21 \mathrm{~V} / \mathrm{m}$ \\
\hline
\end{tabular}

$$
\begin{gathered}
\mu_{e p}=\frac{q}{6 \pi \eta R}=6.35 \times 10^{-12} \mathrm{~m}^{2} / V \cdot s \\
V_{e p}=\mu_{e p} E=8.159 \times 10^{-9} \mathrm{~m} / \mathrm{s}=8.159 \times 10^{-3} \mu \mathrm{m} / \mathrm{s}
\end{gathered}
$$

After calculating the two velocities, it was clear that there is was a large difference between the experimental and calculated values. With this knowledge, it is very difficult to determine if the CE system was effective in transporting the spheres down the channel. The images of the spheres with the shifting voltages were then analyzed to determine if the spheres were driven by voltage.

The second test allowed the spheres to settle more and allow the residual effects of the pressure of injection to diminish. The voltage was reversed two times, causing the spheres to reverse directionality each time the voltage changed. Figure 49 shows the spheres flowing from left to right in the channel in row A, then reversing the polarity so the spheres flowed from right to left in row B, and finally reversing to the original polarity of left to right in row $\mathrm{C}$. 


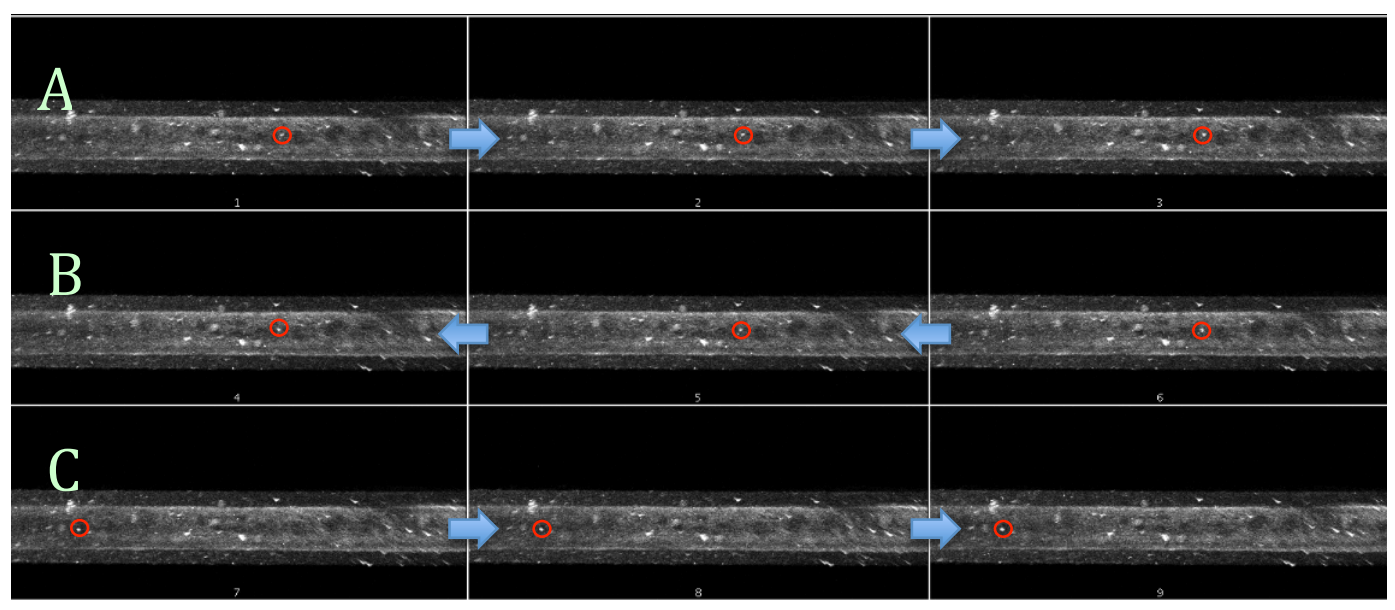

Figure 49. Voltage-driven flow of 10 um spheres shifted by reversing the polarity of the electrodes with A) spheres flowing from left to right in the channel, B) reversing polarity so spheres flowed from right to left, and C) final polarity shift with spheres traveling from left to right.

The absolute value of 200 distances values were recorded and the average velocity was calculated in the same way as previously described (Table 9). The velocity of the spheres due to the voltage was closer to the theoretical value than the first experiment with the syringe in place and immediately after the pressure was applied.

Table 9. Table of values for voltage-driven flow of 10 um microspheres.

\begin{tabular}{|c|c|c|}
\hline & Average & Standard Deviation \\
\hline Distance $(\mu \mathrm{m})$ & 2.438 & 1.151 \\
\hline $\begin{array}{c}\text { Velocity } \\
(\mu \mathrm{m} / \mathrm{sec})\end{array}$ & 11.025 & 5.203 \\
\hline $\begin{array}{c}\text { Theoretical } \\
\text { Velocity } \\
(\mu \mathrm{m} / \mathrm{sec})\end{array}$ & $8.159 \times 10^{-3}$ & \\
\hline Percent Error & $1350.27 \%$ & \\
\cline { 1 - 2 } & &
\end{tabular}


Potential differences in velocity between theoretical and calculated values could be due to a variety of reasons. One main reason for the difference could be that the residual pressure from the injection of the spheres continued to move the spheres and overcame the velocity created by the voltage. Another potential reason could be a form of vacuum is created on the input end when the spheres are injected leading to the spheres being moved due to a difference in pressure on either end even after the pressure is released. If the syringe is removed, the pressure can equalize over time and the resulting movement would be due to the applied voltage.

The next experiment replaced the microspheres with an MDA solution and the experiment was performed again in order to see if the CE system would be successful in driving the MDA particles down the channel.

MDA

The next experiment was designed to validate the proof-of-concept CE system to detect MDA in order to determine if it was feasible to proceed with the design.

A new syringe was filled with the solution of MDA prepared in the method previously discussed. An arbitrary location of the channel was selected and imaged prior to injection as a control. The syringe was then inserted into the 
port and slight pressure was applied by hand to inject the MDA into the channel. Images were taken without voltage. The voltage supply was then turned on to $40 \mathrm{~V}$ with the cathode in the waste well and the anode in the buffer well. The voltage was then turned up to $48.8 \mathrm{~V}$, the maximum voltage possible on the given voltage source, to increase the speed at which the MDA would reach the field of view. The sample then flowed past the point of interest with a parabolic shape. The parabolic shape was unusual as capillary electrophoresis is supposed to flow as a flat shape and parabolic plug are produced by pressure driven flow. The voltage was then turned off and some flow was still seen. This flow could be due to residual effects of the electrical signal or differences in pressure along the channel. The channel was then cleaned with deionized water injected by a syringe.

ImageJ Results \& Analysis

The following (Figure 50 through Figure 55) are selected images from the sequence where the voltage was applied and flow was seen with a parabolic shape. The first image shows the channel after being washed with deionized water, before the MDA enters the channel (Figure 50). 


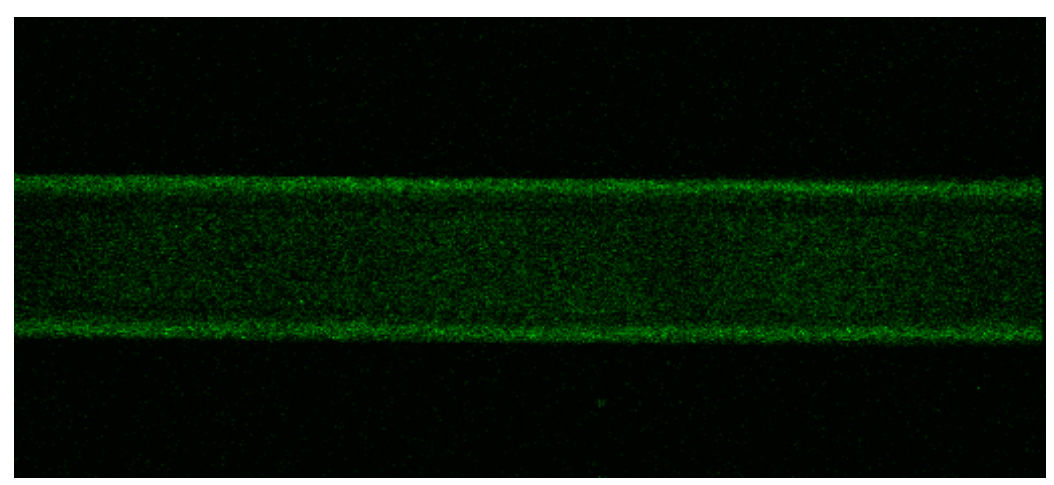

Figure 50. Slice 1 of 200 showing the microchannel with no MDA present.

The next image shows the MDA solution filling the entire channel in the field of view as seen by the vibrant green color (Figure 51). The leading and trailing end of the plug is not visible therefore it cannot be determined if the plug is driven by pressure or voltage. The velocity of the MDA solution was greater than the scan rate of the frame thus the leading portion of the MDA was not imaged.

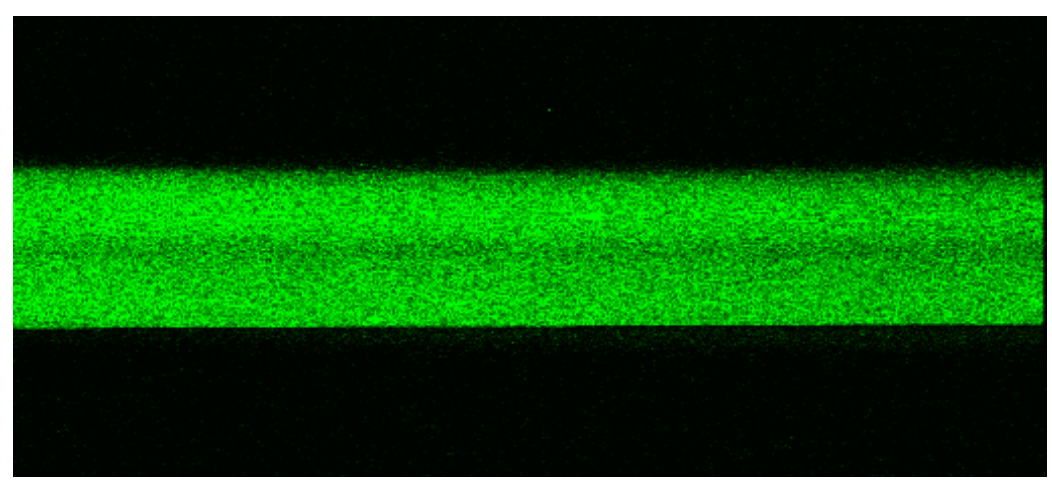

Figure 51. Slice 20 of 200 showing the presence of MDA known by the increased intensity of the fluorescence in the channel.

Next, the MDA can be seen in the field of view of the channel with parabolic flow seen in the front and back of the MDA plug (Figure 52). The parabolic shape 
could due to an air bubble in the channel resulting from the pressure from the syringe injection. The parabolic shape of the MDA in the channel could also be due to pressure-driven flow. However the most likely scenario is that there were air bubbles in the channel because the parabola at the leading end of the plug is facing the wrong direction if it was driven by pressure.

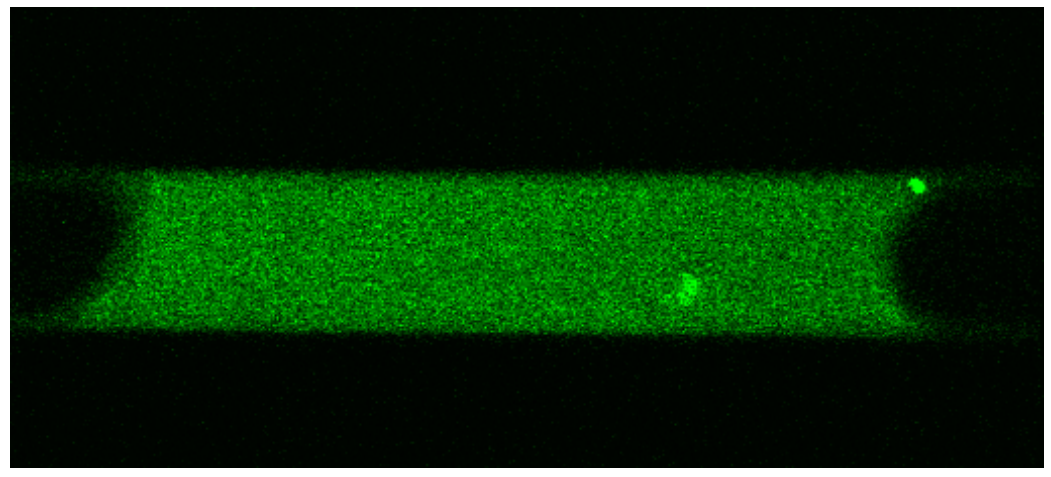

Figure 52. Slice 23 of 200 with MDA present with parabolic flow leading and following the fluid.

In the next image, the MDA can again be seen in the field of view of the channel with parabolic flow seen in the front of the MDA plug showing that the MDA continued down the channel with an air bubble on either side of the MDA plug (Figure 53). 


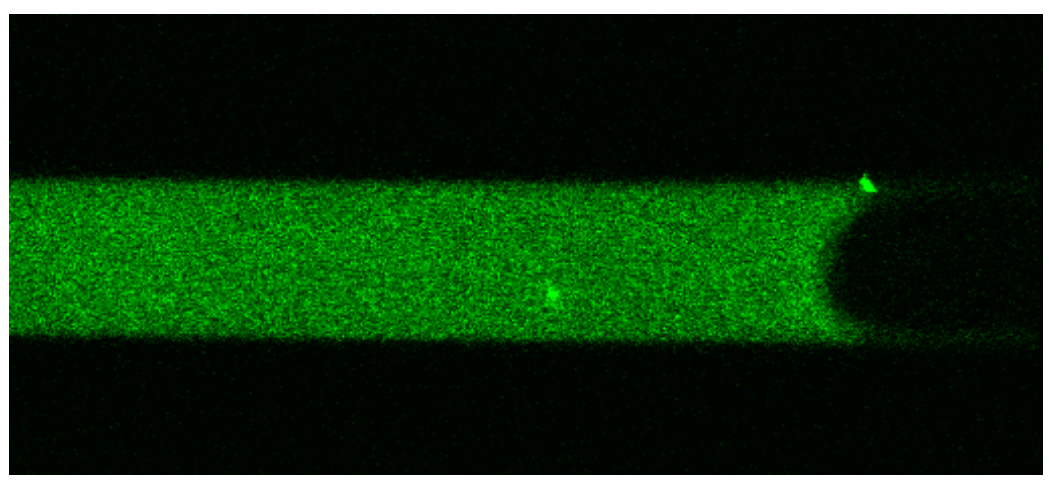

Figure 53. Slice 24 of 200 with MDA present with parabolic flow following the fluid.

Following plug, the MDA can again be seen in the field of view of the channel with a sharp half-parabolic flow seen in the front of the MDA plug showing that the MDA continued down the channel with an air bubble present in the channel (Figure 54).

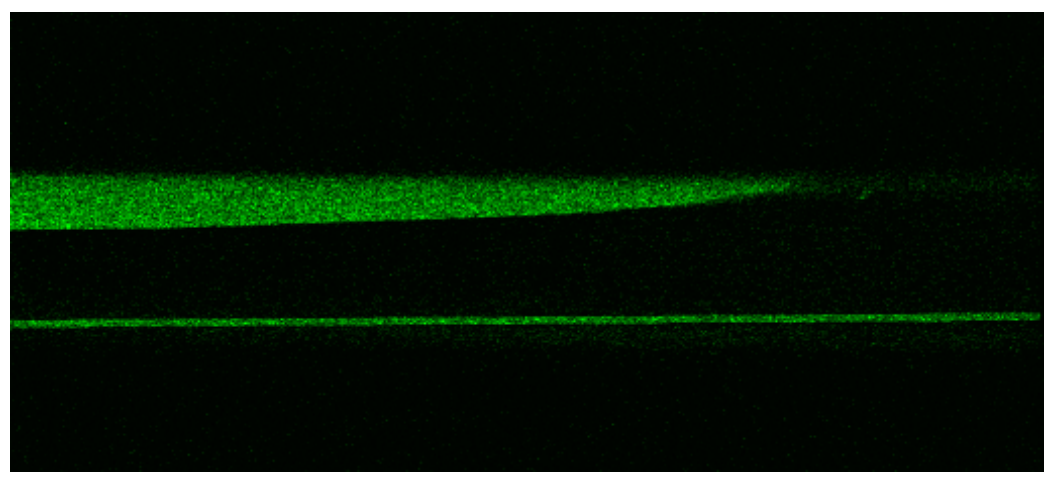

Figure 54. Slice 25 of 200 with MDA present in the top left corner of the channel in the region of interest, with a parabolic shape following the fluid.

Finally, the last image has the MDA solution filling the entire channel in the field of view as seen by the vibrant green color (Figure 55). The leading and trailing 
end of the plug is not visible therefore it cannot be determined if the plug is driven by pressure or voltage.

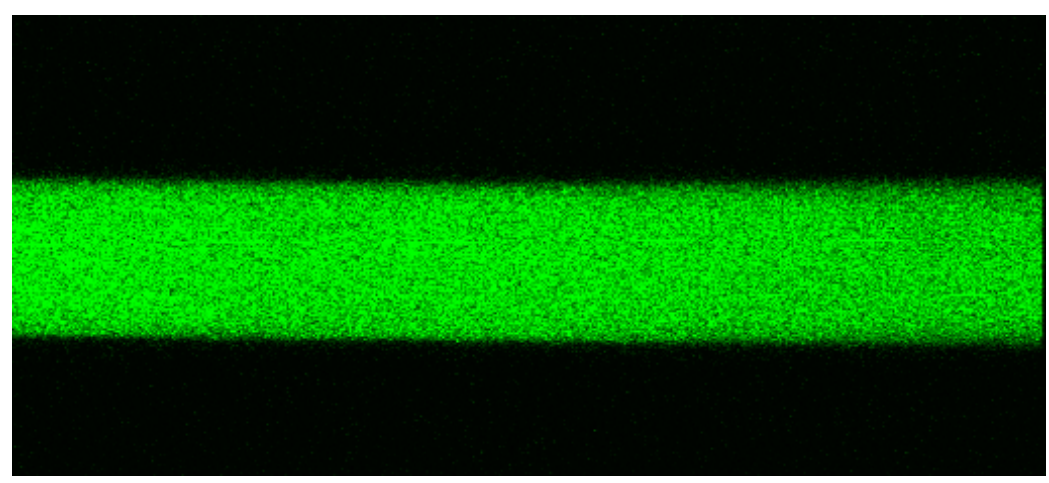

Figure 55. Slice 28 of 200 with MDA present in the microchannel as seen by the increased intensity of fluorescence.

After viewing the flow of MDA in the channel, the velocity of the MDA was determined using ImageJ. The stack was separated into substacks if needed by following the steps previously described. The stacks or substacks were then separated into separate images in order to measure the distance traveled by the individual spheres. The individual images were then overlaid with one image over another and a line was drawn between the areas of MDA in two locations (Figure $56)$. 


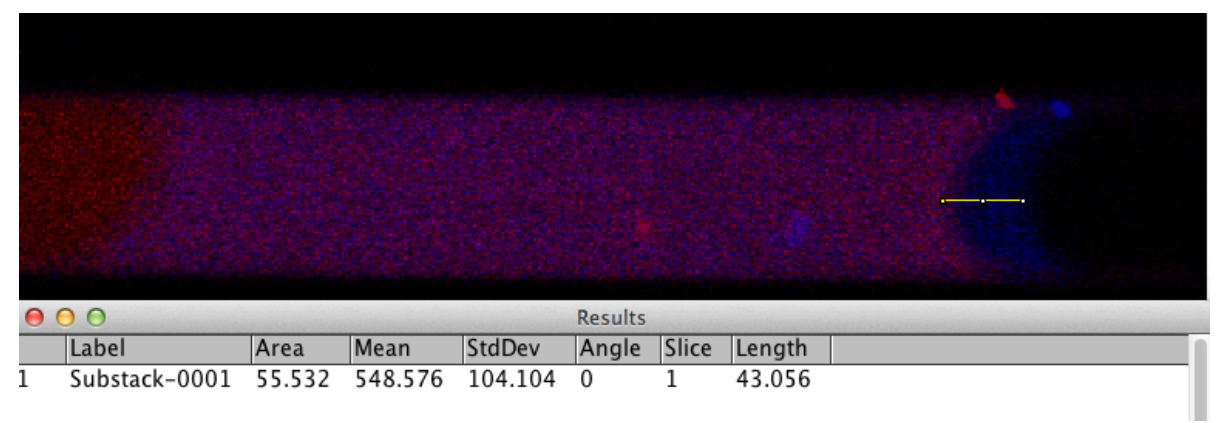

Figure 56. Measuring the distance traveled by the MDA solution in the channel.

A total of 5 distance values were determined from the overlaid images and were then entered into Microsoft Excel to calculate the average and standard deviation of the data. The time between frames was then calculated by multiplying the scan rate of the image by the size of the image. Next, the distance traveled per frame was divided by the time between frames in order to calculate the velocity at which the spheres traveled. Finally, the average and standard deviation of the data was taken and recorded (Table 10).

$$
\begin{gathered}
\text { Image size }\left(\frac{\text { pixel }}{\text { frame }}\right) \times \text { Scan Rate }\left(\frac{\mu \text { sec }}{\text { pixel }}\right) \\
=\text { Time Between Frames }\left(\frac{\mu s e c}{\text { frame }}\right) \\
\left(512 \times 228 \frac{\text { pixel }}{\text { frame }}\right) \times\left(\frac{2 \mu \text { sec }}{\text { pixel }}\right)=233472 \frac{\mu \text { sec }}{\text { frame }}=0.233472 \frac{\mathrm{sec}}{\text { frame }}
\end{gathered}
$$


Table 10. Table of values for voltage-driven flow of MDA solution.

\begin{tabular}{|c|c|c|}
\hline Distance $(\mu \mathrm{m})$ & 38.67 & 3.99 \\
\hline $\begin{array}{c}\text { Velocity } \\
(\mu \mathrm{m} / \mathrm{sec})\end{array}$ & 165.62 & 17.08 \\
\hline $\begin{array}{c}\text { Theoretical } \\
\text { Velocity } \\
(\mu \mathrm{m} / \mathrm{sec})\end{array}$ & 141.9 & \\
\hline Percent Error & $16.72 \%$ & \\
\hline
\end{tabular}

Calculating the velocity of the MDA traveling down the channel allowed for a comparison between the calculated velocity versus the experimental values in order to better understand the experimental CE system. The calculated velocity was calculated using the Debye-Huckel-Henry theory to calculate the electrophoretic mobility, $\mu_{\mathrm{ep}}$, which was used to calculate the electrophoretic velocity, $\mathrm{v}_{\mathrm{ep}}[36]$ (Table 11).

Table 11. Variables used to calculate velocity of voltage-driven MDA.

\begin{tabular}{|l|l|l|}
\hline Variable & Definition & Assumed Value \\
\hline $\mathrm{q}$ & Net charge of particle & $2.4 \times 10^{-18} \mathrm{C}$ \\
\hline $\mathrm{R}$ & Stokes radius of particle & $1.15 \times 10^{-9} \mathrm{~m}$ \\
\hline$\eta$ & Viscosity of solution & $1.002 \mathrm{mPa}-\mathrm{s}$ \\
\hline $\mathrm{E}$ & Electric Field Strength & $1284.21 \mathrm{~V} / \mathrm{m}$ \\
\hline \multicolumn{3}{|c|}{$\mu_{e p}=\frac{q}{6 \pi \eta R}=1.105 \times 10^{-7} \mathrm{~m}^{2} / \mathrm{Vs}$} \\
$V_{e p}=\mu_{e p} E=1.482 \times \frac{10^{-4} \mathrm{~m}}{\mathrm{~s}}=141.899 \mu \mathrm{m} / \mathrm{s}$
\end{tabular}


There was some variation between experimental and calculated velocities for the MDA solution. The CE system needs to be further analyzed in order to explore the areas of variability that may have caused the discrepancy in velocity of the MDA fluid prior to next generation development. 


\section{Discussion and Conclusion}

Traumatic brain injuries are a large problem especially for military personnel. Currently there is a need for field detection of TBIs in order to diagnose patients earlier to increase their care and ultimately quality of life. TBIs produce a cascade that includes an increase in production of reactive oxygen species leading to an oxidative state in the body. This oxidative state can cause further injuries especially to the brain, such as neuronal degeneration. There are many types of ROS found when the body is in an oxidative state including malondialdehyde. MDA can be found in many bodily specimens including plasma in blood, and it can be detected in a variety of methods including capillary electrophoresis. Current technologies for CE include lab-on-a-chip or a microfluidic chip.

Pressure-Driven Flow on Confocal Microscope

The test for pressure-driven flow of microspheres was designed to confirm an accurate fluid flow from the sample well to the sample waste well through the sample and separation channels. Viewing the microspheres flow in and out of the field of view demonstrated that the fluid could flow uninhibitly through the sample and separation channels. ImageJ was then used to calculate the velocities at which the spheres traveled through the field of view from pressure-driven flow. The average velocity of spheres traveling down the channel was $397.22 \mu \mathrm{m} / \mathrm{sec}$ however the standard deviation was $310.22 \mu \mathrm{m} / \mathrm{sec}$. The large standard deviation 
could be due to the different paths the spheres traveled. Some of the spheres traveled faster than the scanning speed therefore their distance could not be calculated, while others may have bounced off the sides of the channel causing variations in velocities. Once it was determined that fluid could flow through the channels and the confocal microscope could view the channel, MDA was then exchanged for the microspheres.

The MDA solution was then used for pressure-driven flow through the chip in order to verify that the fluid could flow through the sample and separation channels between the sample well and sample waste well. In addition, the experiment demonstrated that the MDA solution could be detected using a confocal microscope and that pressure could be used to drive the fluid through the channels if the voltage failed during $\mathrm{CE}$. The average velocity of MDA with pressure-driven flow was estimated to be $2192.98 \mu \mathrm{m} / \mathrm{sec}$. This is an inaccurate estimate due to the inability to detect a specific location to track over time within the fluid flow. The failure to detect a specific location could be due to the fluid flowing at a greater velocity than that of the scan speed of the confocal microscope or due to the plug flowing longer than the field of view. Without two specific points over time, it was impossible to calculate the experimental velocity of the MDA solution due to pressure-driven flow. Although the exact velocity could not be determined, the fluid was seen flowing through the field of view, 
which verified that the MDA flowed through the sample and separation channels between the sample and sample waste wells.

It should be noted that one source of error, for all of the experiments in this study, is human error. The locations selected in ImageJ, used to calculate the distance traveled by the spheres or MDA solution, were locations determined by a person and therefore not precise. From the results from the pressure-driven flow experiments, it can be concluded that pressure could be used to drive the system if the CE system fails.

\section{Voltage-Driven Flow on Confocal Microscope}

The test for voltage-driven flow of microspheres on the confocal microscope was to learn if the CE setup was suitable and determine a procedure for the experimental CE system. The microsphere sample was injected into the sample channel from the sample well and the electric field was induced between the sample and sample waste wells. The spheres were observed entering and exiting the field of view on the confocal microscope demonstrating that the spheres flowed down the channel. From this observation, it was assumed that the CE system successfully created an electric field which drove the spheres down the channel. The average velocity of the spheres during this experiment was 326.05 $\mu \mathrm{m} / \mathrm{sec}$ which was significantly greater than the theoretical velocity of 0.0082 $\mu \mathrm{m} / \mathrm{sec}$. The disparity between the theoretical and experimental velocities could 
be a result of the spheres continuously being driven by pressure even though the pressure source had been removed for an extended period of time. After comparing the average velocities from experimental data between pressure-driven and voltage-driven flow, $397.22 \mu \mathrm{m} / \mathrm{sec}$ as compared to $326.05 \mu \mathrm{m} / \mathrm{sec}$ respectively, the conclusion could be drawn that the spheres flowed down the channel due to the applied pressure from the syringe.

Conversely, the second experiment for the voltage-driven flow of microspheres, demonstrated that voltage played a large role in the movement of the particles. The average velocity of the spheres during the voltage shifting experiment was $11.025 \mu \mathrm{m} / \mathrm{sec}$ which is closer to the theoretical value of 0.0082 $\mu \mathrm{m} / \mathrm{sec}$. By removing the syringe and allowing more time to pass, the particles were able to settle in the channel and the effects of the residual pressure decreased. However, if the spheres wait too long, the spheres will settle on the bottom of the channel which can be difficult to move and may require the pressure to be applied again. After the second experiment, it could be concluded that the applied voltage played a large role in the movement of the spheres after allowing enough time for the residual pressure to dissipate.

The purpose of the voltage-driven flow of MDA on the confocal microscope was to validate the proof-of-concept CE system to determine if the model was feasible to proceed. The MDA solution was injected into the sample channel from the sample well and the electric field was induced between the 
sample and sample waste wells. The average velocity of the MDA during this experiment was $165.62 \mu \mathrm{m} / \mathrm{sec}$ which was greater than the theoretical velocity of $141.9 \mu \mathrm{m} / \mathrm{sec}$. The disparity between the theoretical and experimental velocities could be a result of the MDA continuing being driven by pressure even though the pressure source had been removed for an extended period of time. The significantly smaller size of the MDA particles when compared to the spheres, make it impossible to track any single MDA particle to determine a more precise velocity. The only way it was possible to calculate the velocity of the MDA solution was due to the parabolic shapes leading and following plugs created by air bubbles in the system. To reduce these air bubbles and allow for continuous flow, the syringe can be void of all air bubbles prior to attaching it to the system. In addition, the channel can be completely flushed with the MDA solution prior to removing the syringe to remove any air bubbles from the channel.

The assumed conclusion from the project is that the proof-of-concept $\mathrm{CE}$ system was successful after the pressure from the injection subsided and allowed the spheres and MDA to flow down the channel during all tests. It should be noted that the theoretical velocity was calculated using assumed values. These values, such as viscosity of the buffer solution, vary depending on a variety of factors including temperature of the solution which is increased as the voltage is applied for extended periods of time. One problem with the current experiments was that enough time did not pass after the pressure was used to inject the fluid into the 
channel. If the fluid had time to decrease velocity prior to the application of the electric field, the fluid would then be purely driven by voltage. On the other hand, an increased voltage would either accelerate the fluid to a velocity greater than that caused by pressure or maintain the velocity of the fluid that was created by pressure. There are many risks associated with an increased voltage, which can make the system dangerous for the user. From these conclusions, it can be discerned that further experimentation and development is necessary to determine the feasibility of a portable TBI detection device. 
Future Work and Recommendations

There are many areas for future improvement and development of the current work in order to create a portable TBI detection device. The following describes a few suggestions on improvements of the experiments performed in this study.

Pressure-Driven Flow on Confocal Microscope

The pressure-driven flow of both the spheres and the MDA solution could have been explored more thoroughly in order to better understand the equipment prior to beginning experiments with voltage-driven flow. The pressure to the syringe, used to inject the solutions into the channel of the chip, can be applied using a syringe pump therefore knowing an exact pressure to calculate an approximate velocity of the fluids as they flow down the channel. With the syringe, removing all air bubbles from the syringe and the channel will help in the voltage-driven portion so the air bubbles do not disrupt the flow of fluids through the channel. Another area of improvement would be to use a different solution for the run buffer, as opposed to the deionized water. Observing the fluid flow of different buffer solutions, such as any from the articles found in Table 2, with a known applied pressure could be used as a form of control for the voltage experiments with the same buffer solutions. Finally, flowing fluid through the buffer and buffer waste wells with pressure-driven flow could help characterize the buffer sample channels to use with voltage-driven flow for later experiments. 
Characterizing the microchip with pressure-driven flow provides an avenue that may be considered a better method of analysis of MDA detection in a sample than capillary electrophoresis.

\section{Voltage-Driven Flow on Confocal Microscope}

From the data, it was cannot be concluded that the voltage-driven flow was successful or unsuccessful in this study therefore allowing for a multitude of improvements. First, duplicating one to two experiments from the studies found in Table 2 with the microchip, can aid in better understanding the process of capillary electrophoresis. In order to follow the protocols, the run buffer solution described in the experiment would be used in place of the deionized water as the buffer has proven success in prior experiments. In addition to the buffer, the high voltage would need to be applied to the system to induce the high electric field. The high voltage continues the movement of the solution down the channel after the pressure is removed from injecting the samples into the channel. Increasing the voltage to the kilovolt range, should mimic the experiments described in the papers, Table 2, which have been concluded to have successfully demonstrated CE. Furthermore, the conditions should be tested with a tabletop system prior to being injected into the microchip to verify that the buffer and voltages effectively transport the particles down the capillary. Finally, the injection method of fluids into the channel needs to be standardized possibly by using a syringe pump in order to have reproducible results and a precise protocol. An area of the injection 
method that needs to be explored further is the time required to wait between removing the syringe, after injecting the solution into the channel, before the flow is driven by voltage. From the experiments performed in this study, it was seen that a length of time must be waited for the residual effects of the pressure to diminish in order for the voltage to successfully drive the solution down the channel. The time cannot be for an extended time as the particles can settle to the bottom of the channel and will not be moved until pressure is applied to the system. Once these areas of the CE system are understood, optimization and alterations of the buffer solution, applied voltage and injection methods can be made to make the system better equipped for field applications.

\section{Blood Separation}

Once the separation CE system is understood and successful, and permission is granted, development of the blood separation system can be attempted ass it could not be during this study. The microchip blood separation technique provides a methodology that could easily be replicated and integrated onto a chip with the CE system (Figure 57). Recreating the techniques described in the paper could be a fairly simple approach with areas for success. If the technique was successful, integration of the two chip designs could be possible by using the plasma waste reservoir as the sample well for the CE system. Prior to integration of the systems, a test to validate that MDA remains in the plasma after separation would be necessary. Once proven, the blood separation portion could 
be optimized to determine the required volume of whole blood needed to obtain enough plasma for an accurate reading of MDA concentration in the sample.

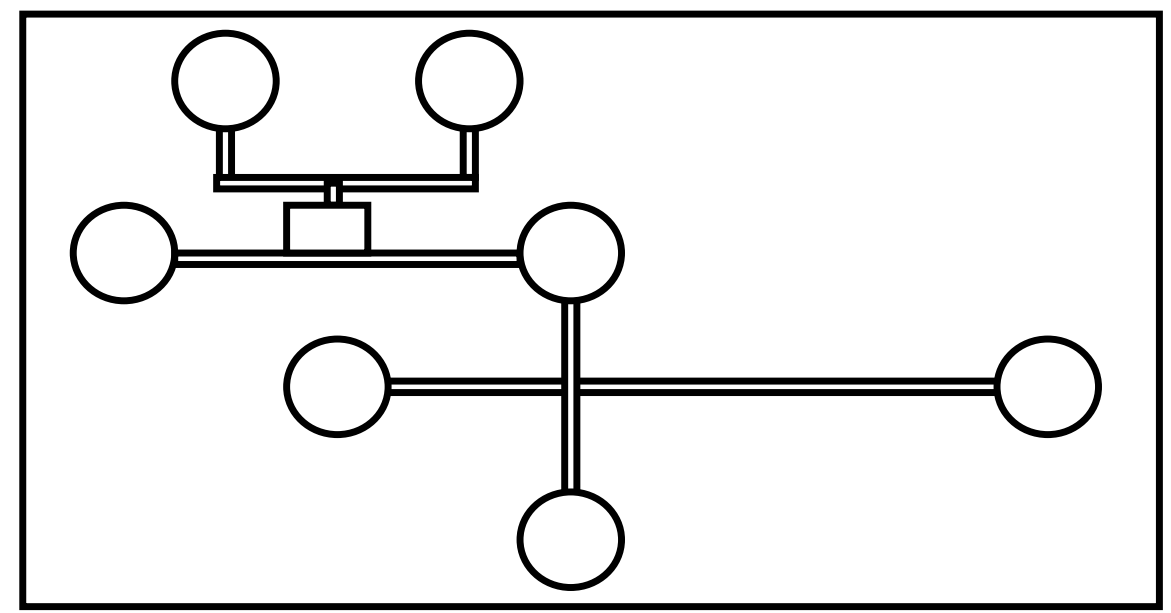

Figure 57. Possible design for microchip combining both whole blood separation and MDA detection.

Once the system has been optimized, the accuracy or limit of detection needs to be determined and compared to clinical values. If the limit of detection were too low to detect a difference between an oxidative and non-oxidative state, the system would be futile. The system could help by creating a standard for detection of MDA in relation to TBI by reducing the variances and inconsistences due to methodologies of sample preparation and analysis techniques. Using a standard procedure a baseline for normal and injured states could be created. Relating time, injury types, and outcomes would greatly improve clinical practice of treating TBIs by developing procedures based on the known outcome from a MDA reading. The potential to reduce TBI effects from early diagnosis would greatly improve the quality of life of patients globally and could change the 
outcome of patients from unfavorable to favorable and reduce the costs of TBI related problems. 


\section{BIBLIOGRAPHY}

1. "Injury Prevention \& Control: Traumatic Brain Injury." Centers for Disease Control and Prevention. Centers for Disease Control and Prevention, 15 Aug. 2013. Web. 14 Apr. 2013.

2. M., Arand, Melzner H., Kinzl L., Brückner U., and Gebhard F. "Early Inflammatory Mediator Response following Isolated Traumatic Brain Injury and Other Major Trauma in Humans." Langenbeck's Archives of Surgery 386.4 (2001): 241-48. Print.

3. Mayo Clinic Staff. "Traumatic Brain Injury." Mayo Clinic. Mayo Foundation for Medical Education and Research, 12 Oct. 2012. Web. 14 Apr. 2013.

4. Marieb, Elaine Nicpon, and Katja Hoehn. Anatomy \& Physiology. 4th ed. Upper Saddle River, NJ: Pearson/Benjamin Cummings, 2011. Print.

5. Jeffrey V Rosenfeld, Andrew I Maas, Peter Bragge, M Cristina MorgantiKossmann, Geoffrey T Manley, Russell L Gruen, Early management of severe traumatic brain injury, The Lancet, Volume 380, Issue 9847, 22-28 September 2012, Pages 1088-1098, ISSN 0140-6736, http://dx.doi.org/10.1016/S0140-6736(12)60864-2. (http://www.sciencedirect.com/science/article/pii/S0140673612608642 )

6. Paolin, Adolfo, Lorella Nardin, Paolo Gaetani, Riccardo Rodriguez Y Baena, Orietta Pansarasa, and Fulvio Marzatico. "Oxidative Damage after Severe Head Injury and Its Relationship to Neurological Outcome." Neurosurgery 51.4 (2002): 949-55. PubMed. U.S. National Library of Medicine \& National Institutes of Health. Web. 6 Mar. 2013.

7. Cohen, Stewart L., Esq. "Symptoms of Traumatic Brain Injury (TBI)." TraumaticBrainInjurycom. Ed. David Lenrow, M.D. and Joanne Finegan, MSA, CTRS. TraumaticBrainInjury.com, LLC, 2004. Web. 30 Jan. 2013.

8. Glasgow Outcome Scale. N.p.: Http://www.tbiimpact.org/cde/mod_templates/12_F_01_GOSE.pdf, n.d. PDF.

9. Held, Paul. "An Introduction to Reactive Oxygen Species - Measurement of ROS in Cells." Tech Resources - App Guides. BioTek Instruments, Inc, 23 Feb. 2010. Web. 8 Nov. 2012. 
10. Awasthi, Deepak, M.D., Daniel F. Church, PhD., Dan Torbati, PhD., Michael E. Carey, M.D., and William A. Pryor, PhD. "Oxidative Stress following Traumatic Brain Injury in Rats." Surgical Neurology 47.6 (1997): 575-81. Surgical Neurology. Elsevier Inc., June 1997. Web. 23 Jan. 2013.

11. Finkel, Toren, and Nikki J. Holbrook. "Oxidants, Oxidative Stress and the Biology of AgeingToren Finkel and Nikki J. Holbroo." Nature.com. Nature Publishing Group, 9 Nov. 2000. Web. 9 June 2013.

12. Mylonas, C., and D. Kouretas. "Lipid Peroxidation and Tissue Damage." In Vivo 13.3 (1999): 295-309. Europe PubMed Central. Europe PMC Founder's Group, 13 June 1999. Web. 10 June 2013.

13. Rio, Daniele Del, Amanda J. Stewart, and Nicoletta Pellegrini. "A Review of Recent Studies on Malondialdehyde as Toxic Molecule and Biological Marker of Oxidative Stress." Nutrition, Metabolism and Cardiovascular Diseases 15.4 (2005): 316-28. Elsevier. Science Direct, 2005. Web. 30 May 2013.

14. Pryor, William A., and J. P. Stanley. "Suggested Mechanism for the Production of Malonaldehyde during the Autoxidation of Polyunsaturated Fatty Acids. Nonenzymic Production of Prostaglandin Endoperoxides during Autoxidation." The Journal of Organic Chemistry 40.24 (1975): 3615-617. The Journal of Organic Chemistry. ACS Publications. Web. 10 June 2013.

15. Esterbauer, Hermann, Rudolf Jörg Schaur, and Helmward Zollner. "Chemistry and Biochemistry of 4-hydroxynonenal, Malonaldehyde and Related Aldehydes." Free Radical Biology and Medicine 11.1 (1991): 81-128. Elsevier. Science Direct. Web. 10 June 2013.

16. Bastos, Alliny Souza, Ana Paula De Melo Loureiro, and Tiago Franco De Oliveira. "Quantitation of Malondialdehyde in Gingival Crevicular Fluid by a High-performance Liquid Chromatography-based Method." Analytical Biochemistry 423.1 (2012): 141-46. Eleviser. Science Direct, 2012. Web. 10 June 2013.

17. Dean, Laura. "Blood and the Cells It Contains." Blood Groups and Red Cell Antigens. Bethesda, MD: NCBI, 2005. N. pag. NCBI. Web. 9 June 2013.

18. Moselhy, Hamdy F., Raymond G. Reid, Saeed Yousef, and Susanne P. Boyle. "A Specific, Accurate, and Sensitive Measure of Total Plasma Malondialdehyde by HPLC." Journal of Lipid Research 54.3 (2013): 852-58. Journal of Lipid Research. 20 Dec. 2012. Web. 10 June 2013. 
19. "Vivid" ${ }^{\mathrm{TM}}$ Plasma Separation Membrane." Vivid ${ }^{\mathrm{TM}}$ Plasma Separation Membrane. Pall Corporation, n.d. Web. 12 Mar. 2013.

20. Jiang, Hai, Xuan Weng, Chan Hee Chon, Xudong Wu, and Dongqing Li. "A Microfluidic Chip for Blood Plasma Separation Using Electroosmotic Flow Control." Journal of Micromechanics and Microengineering 21.8 (2011): n. pag. Journal of Micromechanics and MicroengineeringJournal of Micromechanics and Microengineering. IOP Publishing, 11 July 2011. Web. 2 June 2013.

21. Lewis, A. P., A. Cranny, N. R. Harris, N. G. Green, and J. A. Wharton. "Review on the Development of Truly Portable and In-situ Capillary Electrophoresis Systems." Measurement Science and Technology 24.4 (2013): n. pag. IOP Science. IOP Publishing. Web. 9 Apr. 2013.

22. Glynn, J. R., Jr., B. M. Belongia, R. G. Arnold, K. L. Ogden, and J. C. Baygents. "Capillary Electrophoresis Measurements of Electrophoretic Mobility for Colloidal Particles of Biological Interest." APPLIED AND ENVIRONMENTAL MICROBIOLOGY 64.7 (1998): 2572577. APPLIED AND ENVIRONMENTAL MICROBIOLOGY. American Society for Microbiology. Web. 10 June 2013.

23. Probstein, Ronald F. Physicochemical Hydrodynamics: An Introduction. 2nd ed. Hoboken, NJ: Wiley-Interscience, 2003. Print.

24. Paroni, Rita, Isabella Fermo, and Giuliana Cighetti. "Validation of Methyl Malondialdehyde as Internal Standard for Malondialdehyde Detection by Capillary Electrophoresis." Analytical Biochemistry 307.11 (2002): 92-98. Elsevier. Science Direct. Web. 23 Jan. 2013.

25. Zinellu, Angelo, Salvatore Sotgia, Luca Deiana, and Ciriaco Carru. "Fieldamplified Sample Injection Combined with Pressure-assisted Capillary Electrophoresis UV Detection for the Simultaneous Analysis of Allantoin, Uric Acid, and Malondialdehyde in Human Plasma." Analytical and Bioanalytical Chemistry 399.8 (2011): 2855-861. Springer Link. Web. 10 June 2013.

26. Wilson, David W., Helene N. Metz, Michael L. Graver, and Parinam S. V. "Direct Method for Quantification of Free Malondialdehyde with High-Performance Capillary Electrophoresis in Biological Samples." Clinical Chemistry 43.10 (1997): 1982-984. Clinical Chemistry. American Association for Clinical Chemistry. Web. 10 June 2013.

27. Korizis, K. N., Exarchou, A., Michalopoulos, E., Georgakopoulos, C. D., Kolonitsiou, F., Mantagos, S., Gartaganis, S. P. and Karamanos, N. K. (2001), "Determination of malondialdehyde by capillary 
electrophoresis, application to human plasma and relation of its levels with prematurity." Biomedical Chromatography, 15.4 (2001) 287-291. John Wiley \& Sons, Ltd. Web. 10 June 2013.

28. Cronk, Terri Moon. "Military Leads in Treating Traumatic Brain Injury, Expert Says." United States Department of Defense. United States Department of Defense, 31 Aug. 2012. Web. 10 June 2013.

29. Cooley, Justin Carl, and Craig Edward Lunte. "Detection of Malondialdehyde in Vivo Using Microdialysis Sampling with CEfluorescence." Electrophoresis 32.21 (2011): 2994-999. Electrophoresis Journal. Wiley Online Library, 27 Oct. 2011. Web. 10 June 2013.

30. Duckworth, Josh L., Jamie Grimes, and Geoffrey S.F. Ling. "Pathophysiology of Battlefield Associated Traumatic Brain Injury." Pathophysiology 20.1 (2013): 23-30. Elsevier. Science Direct, Feb. 2013. Web. 10 June 2013.

31. Jones, Dean P. "Redefining Oxidative Stress." Antioxidants \& Redox Signaling 8.9-10 (2006): 1865-879. Mary Ann Liebert, Inc. U.S. National Library of Medicine, 20 Sept. 2006. Web. 15 Oct. 2013.

32. "HPLC - High Performance Liquid Chromatography." Waters, n.d. Web. 15 Oct. 2013.

33. "Ascentis Express C18, $2.7 \mu \mathrm{m}$ HPLC Columns." Sigma-Aldrich. N.p., n.d. Web. 15 Oct. 2013.

34. "Capillary Tubing for Capillary Electrophoresis." Biotaq Capillary. N.p., 2012. Web. 15 Oct. 2013.

35. "Cross-Channel Chips." Translume, Inc., n.d. Web. 14 Apr. 2013.

36. Introduction to Capillary Electrophoresis. N.p.: Beckman Coulter, 1991. PDF.

37. Laiho, Lily, PhD. "Fluorescence Imaging." Biomedical Imaging Lecture. California Polytechnic State University, San Luis Obispo. Oct. 2012. Lecture. 